\title{
A Detailed Analysis of The Multi-class Classification Problem in Network Intrusion Detection using Resampling Techniques
}

\author{
by
}

\author{
Rahbar Ahsan \\ Supervised by: Dr. Wei Shi \& Dr. Jean-Pierre Corriveau
}

A thesis submitted to

the Faculty of Graduate Studies and Research

in partial fulfilment of

the requirements for the degree of

Master of Computer Science

School of Computer Science

Carleton University

Ottawa, Ontario, Canada

April 2021

Copyright (C)

2021 - Rahbar Ahsan 


\section{Abstract}

Cybersecurity has recently become a significant concern. In particular, machine learning algorithms are known to help identify cyberattacks such as network intrusion. However, common network intrusion datasets are often imbalanced: the normal traffic behaviors constitute the majority of the dataset, while intrusion traffic behaviors form a significantly smaller portion. We conduct a detailed analysis on the impact of different resampling techniques over different machine learning classifiers. We include more advanced resampling techniques, such as Conditional Generative Adversarial Network (CGAN) oversampling, in our study and compare their performance against other oversampling techniques. Also, we propose a novel network intrusion detection solution combining Adversarial Environment Reinforcement Learning (AE-RL) with CGAN. Experimental results show that despite an improvement on F1-score, applying a combination of data level approach and algorithm level approach has less impact than using only data level approach. To further investigate the potential of CGAN-based oversampling, we examine the effect of CGAN on the following classifiers: Naïve Bayes, Multilayer Perceptron, Random Forest and Logistic Regression performed on two different datasets. Based on our experimental results, we do not recommend using CGAN in a dataset with extremely low samples in its minority class(es). Consequently, we also investigate the choice of minority class(es) to be oversampled in similar datasets. Finally, the impact of the number of synthetic samples to be generated on the detection rate is evaluated on two different datasets. 


\section{Acknowledgments}

First and foremost, with my most sincere gratitude, I would like to acknowledge my two supervisors Dr. Wei Shi and Dr. Jean-Pierre Corriveau for their dedication,

patience, encouragement, and support. Without their guidance, knowledge and expertise, this dissertation would have not been possible.

I would also like to thank my family for their encouragement and support for my research interests and educational pursuits. 


\section{Table of Contents}

Abstract $\quad$ ii

Acknowledgments $\quad$ iii

Table of Contents $\quad$ iv

List of Tables viii

List of Figures $\quad$ X

Nomenclature $\quad$ xii

1 Introduction 1

1.1 Intrusion Detection system . . . . . . . . . . . . . . 1

1.1.1 Host Based Intrusion Detection System . . . . . . . . . . . . 2

1.1.2 Network Based Intrusion Detection System . . . . . . . . . . . 2

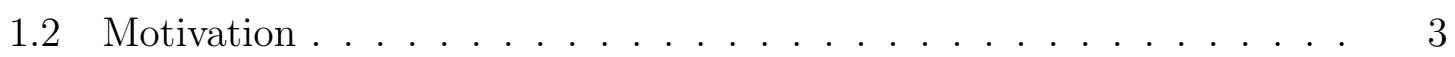

1.3 Problem Statement . . . . . . . . . . . . . . . . . 4

1.4 Thesis Contribution . . . . . . . . . . . . . . . . . 5

1.4.1 List of Publications . . . . . . . . . . . . . . . 9

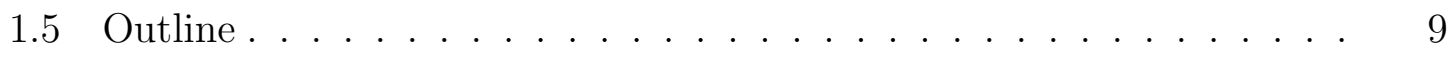

2 Background $\quad 11$ 
2.1 Supervised Machine Learning Algorithms . . . . . . . . . . . . . . . . 11

2.1.1 Decision Tree . . . . . . . . . . . . . . . . 12

2.1.2 Gaussian Naïve Bayes . . . . . . . . . . . . . . . . . . 12

2.1.3 Support Vector Machine . . . . . . . . . . . . . 13

2.1.4 Random Forest . . . . . . . . . . . . . . . . . . . . . 13

2.1.5 Extra Tree . . . . . . . . . . . . . . . 13

2.1.6 Logistic Regression . . . . . . . . . . . . . . . . . 13

2.1.7 Neural Networks . . . . . . . . . . . . . . . . . . . 14

2.1.8 Autoencoder . . . . . . . . . . . . . . . . . . 14

2.2 Reinforcement Learning . . . . . . . . . . . . . . . . 15

2.2.1 Adversarial Environment Reinforcement Learning . . . . . . . 16

2.3 Resampling Techniques . . . . . . . . . . . . . . . . . . 17

2.3.1 Oversampling ..................... 17

2.3.2 GAN as an Oversampling Techniuqe . . . . . . . . . . 18

2.3.3 Undersampling . . . . . . . . . . . . . . . . . . . . 20

2.3.4 Balance sampling . . . . . . . . . . . . . . 21

2.4 Selected Network Intrusion Datasets . . . . . . . . . . . . . . . . 21

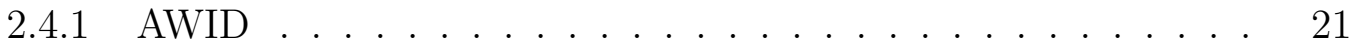

$2.4 .2 \quad$ NSL-KDD . . . . . . . . . . . . . . . . . . . . . 22

2.5 Evaluation Metrics . . . . . . . . . . . . . . . 23

3 Literature Review $\quad 25$

3.1 Work-Related to Standard Machine Learning Algorithm . . . . . . . . 25

3.2 Work-Related to Deep Learning Algorithm . . . . . . . . . . . . . 27

3.3 Handling Imbalance Problem in Network Intrusion Detection . . . . . 29

3.3.1 A Review of Algorithm Level Approach . . . . . . . . . . . . . 29

3.3.2 A Review of Data-Level Approach . . . . . . . . . . . . . 30 
3.3.3 Usage of GAN in The Imbalanced Domain . . . . . . . . . . . 32

4 Comparative Evaluation on Ten Classifiers combined with 3 Resampling Techniques $\quad 34$

4.1 Methodology .......................... 34

4.2 Data Pre-processing . . . . . . . . . . . . . . . . . 35

4.3 Machine Learning Algorithms . . . . . . . . . . . . . . . . . 37

4.3.1 Classical Supervised Machine Learning Algorithms . . . . . . 37

4.3.2 Deep Learning Algorithms . . . . . . . . . . . . . . . . . . . 38

4.4 Results \& Analysis . . . . . . . . . . . . . . . . . . . . 40

5 Study on the Impact of Oversampling Techniques on IDS 50

5.1 Problem Statement \& Solution . . . . . . . . . . . . . . . . . 50

5.2 AEGAN Details . . . . . . . . . . . . . . . . 51

5.3 Experimental Setup . . . . . . . . . . . . . . . . 52

5.4 Experiment Results \& Analysis . . . . . . . . . . . . . . . 55

$6 \quad$ Further Improvement $\quad 64$

6.1 Executing AE-RL on NSL-KDD . . . . . . . . . . . . . 64

6.2 CGAN vs. WCGAN . . . . . . . . . . . . 66

6.3 Variance of The CGAN . . . . . . . . . . . . . . . . 67

6.4 Experiments with The Number of Synthetic Samples . . . . . . . 69

6.5 Results \& Discussion . . . . . . . . . . . . . . . . . . . . . 70

6.5.1 Analysis on the results of AWID Dataset . . . . . . . . . 70

6.5.2 Analysis on the Results of NSL-KDD Dataset . . . . . . . 76

6.6 CGAN Performances on Both Datasets . . . . . . . . . . . . . . 83

7 Conclusion and Future Work $\quad 85$ 
List of References 


\section{List of Tables}

4.1 Comparison of the classifiers on original dataset. . . . . . . . . . . . . 41

4.2 Comparison of the classifiers using SMOTE resampling. . . . . . . . . 43

4.3 Comparison of the classifiers using NearMiss Resampling. . . . . . . . 44

4.4 Comparison of the classifiers using SMOTEENN resampling. . . . . . 46

5.1 Results of AE-RL on AWID . . . . . . . . . . . . . . . 55

5.2 Results of Naive Bayes on AWID . . . . . . . . . . . . . . 57

5.3 Results of MLP on AWID . . . . . . . . . . . . . 58

5.4 Results of Random Forest on AWID . . . . . . . . . . . . . . . 59

5.5 Results of Logistic Regression on AWID . . . . . . . . . . . . . . 60

5.6 Population variance on the F1-scores for each classifier . . . . . . . . 62

6.1 Accuracy and F1-score of CGAN and WCGAN with different classifiers 66

6.2 Standard Deviation of the F1-score of LR on different generator steps (AWID Dataset) . . . . . . . . . . . . . . 68

6.3 Standard Deviation of the F1-score of LR on different generator steps (NSL-KDD Dataset) . . . . . . . . . . . . . . . 68

6.4 Oversampled Minority Classes for a Specific Ratio . . . . . . . . . . 70

6.5 Performance of AE-RL on Different Ratios (AWID) . . . . . . . . . . 71

6.6 Performance of NB on Different Ratios (AWID) . . . . . . . . . 72

6.7 Performance of RF on Different Ratios (AWID) . . . . . . . . . 73

6.8 Performance of MLP on Different Ratios (AWID) . . . . . . . . . . 74 
6.9 Performance of LR on Different Ratios (AWID) . . . . . . . . . 75

6.10 Performance of AE-RL on Different Ratios (NSL-KDD) . . . . . . . . 77

6.11 Performance of NB on Different ratios (NSL-KDD) . . . . . . . . 78

6.12 Performance of RF on Different ratios (NSL-KDD) . . . . . . . . . 79

6.13 Performance of MLP on Different ratios (NSL-KDD) . . . . . . . 80

6.14 Performance of LR on Different ratios (NSL-KDD) . . . . . . . . . . 82

6.15 Results Summary on Three Different Combinations of Oversampling

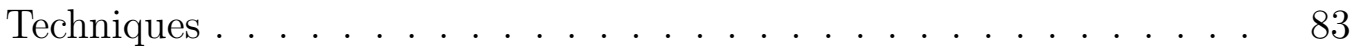




\section{List of Figures}

2.1 Flow of data through the GAN model . . . . . . . . . . . . . . . 19

2.2 Class distribution of AWID training data . . . . . . . . . . . . 22

2.3 Training data distribution of the NSL-KDD dataset. . . . . . . . . 23

4.1 Workflow. . . . . . . . . . . . . . . . . 35

4.2 FCN Architecture. . . . . . . . . . . . . . . . . . . . 38

4.3 Model loss in training and validation datasets in FCN. . . . . . . . . 39

4.4 Architecture of the voting classifier. . . . . . . . . . . . . . . 40

4.5 F1-scores of the classifiers trained on the original dataset. . . . . . . . 42

4.6 F1-scores of the classifiers using SMOTE resampling. . . . . . . . . . 42

4.7 Confusion matrices of Decision Tree classifier trained on the oversampled dataset and the undersampled training sets. . . . . . . . . . 45

4.8 F1-scores of the classifiers using NearMiss resampling. . . . . . . . . . 45

4.9 F1-scores of the classifiers when using SMOTEENN resampling. . . . 47

4.10 F1-scores of the classical machine learning algorithms trained on the original dataset and the datasets generated using 3 resampling techniques. . . . . . . . . . . . . . . . . . . . 47

4.11 F1-scores of the Deep Learning algorithms trained on the original dataset and the datasets generated using 3 resampling techniques. . . 48

4.12 F1-scores of the voting classifier trained on the original dataset and the datasets generated using 3 resampling techniques. . . . . . . . . . 48 
5.1 Algorithm architecture of the detection system . . . . . . . . . . 53

5.2 Various oversample techniques applied on AE-RL . . . . . . . . . 55

5.3 Confusion Matrices of AE-RL \& AEGAN. . . . . . . . . . . . 56

5.4 Various oversample techniques applied to Naive Bayes . . . . . . . . . 57

5.5 Various resample techniques applied to MLP . . . . . . . . . 58

5.6 Various oversampling techniques applied to RF . . . . . . . . . 59

5.7 Various oversampling techniques applied to LR . . . . . . . . . 61

5.8 Confusion Matrices of Logistic Regression with and without CGAN. . 62 


\section{Nomenclature}

\begin{tabular}{l|l}
\hline Abbreviation & Explanation \\
\hline \hline AC & Actor-Critic \\
ADASYN & Adaptive Synthetic Sampling Approach for Imbalanced Learning \\
AdaBoost & Adaptive Boosting \\
AE & Auto-Encoder \\
AE-CGAN & Autoencoder-Conditional GAN \\
AE-RL & Adversarial Environment Reinforcement Learning \\
AEGAN & Adversarial Environment Generative Network \\
AESMOTE & Adversarial Reinforcement Learning with SMOTE \\
ANN & Artificial Neural Network \\
AWID & Aegean WIFI Intrusion Dataset \\
CGAN & Conditional Generative Adversarial Network \\
CVAE & Conditional Variational Auto Encoder (CVAE) \\
CWGAN-GP & Conditional Wasserstein GAN with Gradient Penalty \\
\end{tabular}




\begin{tabular}{|c|c|}
\hline $\mathrm{CNN}$ & Convolutional Neural Network \\
\hline $\mathrm{DBN}$ & Deep Belief Network \\
\hline DDQN & Double Deep Q-Network \\
\hline DDoS & Distributed Denial of Service \\
\hline DNN & Deep Neural Network \\
\hline DoS & Denial of Service \\
\hline $\mathrm{DQN}$ & Deep Q-Network \\
\hline DR & Detection Rate \\
\hline DRL & Deep Reinforcement Learning \\
\hline DSSTE & Difficult Set Sampling Technique \\
\hline EA & Environment Agent \\
\hline ENN & Edited Nearest Neighbor \\
\hline FAR & False Alarm Rate \\
\hline FPR & False Positive Rate \\
\hline GAN & Generative Adversarial Network \\
\hline GMM & Gaussian Mixture Model \\
\hline GRU-RNN & Gated Recurrent Unit-Recurrent Neural Network \\
\hline GVA & Generative Variational Autoencoder \\
\hline HIDS & Host-Based Intrusion Detection System \\
\hline
\end{tabular}




\begin{tabular}{|c|c|}
\hline IDS & Intrusion Detection System \\
\hline IGAN-IDS & Imbalanced Generative Adversarial Network-based Intrusion Detection System \\
\hline $\mathrm{KNN}$ & K-Nearest Neighbour \\
\hline $\mathrm{LR}$ & Logistic Regression \\
\hline LSTM-RNN & Long Short-Term Memory-Recurrent Neural Networks \\
\hline MLP & Multi Layer Perceptron \\
\hline NB & Naïve Bayes \\
\hline $\mathrm{NCM}$ & Normalized Confusion Matrix \\
\hline NIDS & Network Based Intrusion Detection System \\
\hline OCR & Optical Character Recognition \\
\hline ODGAN & Outlier Detectable-GAN \\
\hline $\mathrm{PA}$ & Penalizing Attributes \\
\hline $\mathrm{PCA}$ & Principal Component Analysis \\
\hline $\mathrm{PG}$ & Policy Gradient \\
\hline $\mathrm{PSO}$ & Particle Swarm Optimization \\
\hline $\mathrm{RF}$ & Random Forest \\
\hline RL & Reinforcement Learning \\
\hline RNN & Recurrent Neural Network \\
\hline ROS & Random Over Sampling \\
\hline
\end{tabular}




\begin{tabular}{l|l} 
RUS & Random Under Sampling \\
SAE & Stacked Autoencoder \\
SAVER & Supervised adversarial variational auto-encoder with regularization \\
SMOTE & Single-Objective Generative Adversarial Active Learning \\
SO-GAAL & Support Vector Machine \\
SVM & Wasserstein GAN with Gradient Penalty \\
WGAN-GP
\end{tabular}




\section{Chapter 1}

\section{Introduction}

\section{$1.1 \quad$ Intrusion Detection system}

An exponential rise in the number of computing apps and in the size of networks has drastically increased the potential threat of cyberattacks [1]. It has become essential to ensure network security due to its adaptive nature [2]. Indeed, attackers can intrude on network traffic and control a computer system with various methods. Thus, it becomes essential to anticipate network breaches and identify what kind of intrusion has been attempted to thwart potential intruders [3]. By using numerous types of data sources like reports, network activities, and data gathered from social media and websites, some research institutions and companies are now proposing a model that can predict cybersecurity incidents [4]. One of the proactive strategies to predict cybersecurity incidents is to create an autonomous Intrusion Detection System (IDS) that will help promptly identify and categorize the types of cyberattacks at the network and host infrastructure levels [5]. The key roles of an IDS are tracking hosts and networks, evaluating computer system activities, generating warnings, and reacting to unusual behaviors. IDSs can be classified into two categories based on the source of intrusive behaviors [6], namely: Network Based Intrusion Detection System (NIDS) and Host-Based Intrusion Detection System (HIDS). 


\subsubsection{Host Based Intrusion Detection System}

An IDS system that uses various log files of a host system to find suspicious activities to alert the user is called HIDS. Suspicious activities involve deletion of a system file, intrusive sequence of system calls and unwanted configuration changes [7]. Fine granularity and the ability to track internal attacks are two advantages of HIDS [8]. But a HIDS has disadvantages such as solely depending on the system-generated log files. HIDS cannot work appropriately if systems do not produce logs for any reason. It is also vulnerable to attacks since it is part of a monitored system. If anyone gets access to the host system, they can easily bring down the HIDS server [9].

\subsubsection{Network Based Intrusion Detection System}

An IDS system that exclusively focuses on identifying network based intrusion is called NIDS. NIDS tracks the network traffic for possible intrusion by using various network devices. Important network points are 'covered' by placing network devices like routers to observe the network's flow [10]. Then, NIDS is capable of monitoring many computers connected to the same network, which helps it find the origin of the generated attack. But NIDS has a limitation in performing in a high bandwidth network due to the high volume of information passing through a high-speed communication network [11]. IDS usually calculates thresholds using feature sets that include iter-arrival time, packet length, size of the flow, and other network traffic parameters [12]. These approaches suffer from high false-negative rates and falsepositive rates. This means they have poor performance in detecting the attacks often and create unnecessary false alarms.

Machine learning works well in spam detection, Optical Character Recognition (OCR), and product/video recommendations, but is not as successful for network security. One of the reasons behind this is the lack of public data [13]. Even with the 
availability of a standardized public dataset, machine learning based IDS suffers due to insufficient training data of cyberattacks. But machine learning based IDS can identify intrusion effectively when there is sufficient training data, and the algorithms are flexible enough to identify attack variations and novel threats. In this dissertation, all our work is related to the NIDS using machine learning algorithms.

\subsection{Motivation}

The fact that network intrusion datasets contain large amount of normal data samples and very few attack samples makes these datasets very imbalanced: the normal traffic behavior constitutes the majority of the dataset, while intrusion traffic behavior forms a significantly smaller portion. Class imbalance refers to the disparity between different types of data (e.g., majority of the data samples are in one class and very little in others [14]). The prediction accuracy of machine learning algorithms is greatly affected by the imbalanced nature of a dataset. When making a multi-class classification in detecting intrusive activities, the class imbalance issue significantly reduces the effectiveness of multi-class classifiers, undesirably biasing the results toward the prevailing class, while most of the cyberattacks are in the minority classes. Current research on the classification of imbalanced data is mainly summarized into two types of approaches. The first targets the algorithm-level through the use of classifiers designed with imbalanced data in mind. The objective is to enable the classifier to adapt or strengthen its learning process for the minority classes. An alternative approach involves data-level modifications aimed at balancing the distribution of classes in the training data. Resampling techniques are employed for this purpose and they are divided into oversampling [15], undersampling [16] and hybrid-sampling methods $[17]$.

This dissertation focuses on achieving higher detection accuracy rates by using a 
wide range of resampling techniques on two known imbalanced datasets when performing network intrusion detection. Resampling techniques sometimes increase the false alarm rate. It is challenging to improve the detection rate of cyberattacks with a low false alarm rate. The negative side of a high false alarm rate is that people start to ignore the warning when it frequently occurs. Therefore, our goal is to improve the resampling techniques' efficiency in network intrusion detection by increasing the detection rate of cyberattacks while minimizing the number of false alarms.

\subsection{Problem Statement}

Machine learning-based IDSs have attracted lots of researchers' attention recently. IDSs that utilize machine learning algorithms can identify intrusion effectively when sufficient training data are available, and the algorithms are flexible enough to identify attack variations and novel threats. Furthermore, machine learning-based IDSs are simple to develop and construct without having deep domain-specific knowledge [18]. Several methods focused on classical machine learning have been explored in the past such as Support Vector Machine (SVM) [19, 20], Random Forest [21, 22], KNearest Neighbour (KNN) [23], and Artificial Neural Network (ANN) [24]. Standard machine learning algorithms suffer from the class imbalance problem because their success rests on the balanced distribution of the classes of a dataset of good size. But all known IDS datasets of reasonable sample size have a scarcity of samples that represent cyberattacks. As a new algorithm-level approach, AE-RL [25] was proposed to take advantage of Reinforcement Learning's "signal sensing ability" to solve the dataset's imbalance problem. However, it also suffers from a lack of variations in the minority classes. In this dissertation, we address this issue and provide a solution to this specific problem. In particular, we consider two key factors for exploring various resampling techniques: a) the number of synthetic samples and b) which classes 
are to be oversampled. To the best of our knowledge, no work has been done on creating intrusion detection solutions that take into account these specific factors and perform systematic comparative evaluations on the impact of resampling techniques for intrusion detection.

More specifically, in this dissertation, the research questions we address are as summarized below:

RQ1. What is the impact of the resampling techniques on network intrusion detection systems?

RQ2. Which is more effective: an algorithm-level or a data-level approach or a combination of both?

RQ3. How effective will the combination of an algorithm-level approach with a data-level one be?

RQ4. How effectively can a Generative Adversarial Network (see next section) be used as a data-level approach in a network intrusion detection system?

RQ5. What is the impact of selecting the number of synthetic samples in an oversampling technique?

RQ6. What is the impact of the choice of the class(s) to be oversampled on the final result?

\subsection{Thesis Contribution}

In this dissertation, we mainly focus on addressing the data imbalance problem and providing solutions to the problems that Network Intrusion Detection Systems face. 
First of all, the impact of different resampling techniques on network intrusion detection systems is evaluated. Subsequently, a detailed analysis is presented on oversampling techniques combining with a collection of machine learning algorithms. In order to effectively use the resampling techniques, the impact of the number of synthetic samples and the choices of class(s) are analyzed in detail. To conduct a more comprehensive experimental analysis, some traditional resampling techniques as well as a more complex, non-conventional oversampling technique, the Generative Adversarial Network (GAN)[26] is studied in detail. GAN is a machine learning model that is used to produce novel instances of samples based on a targeted data distribution. GAN is vastly used to generate realistic-looking images. It also gained success in non-image datasets [27]. GAN has much potential to stand out against standard oversampling techniques and contribute to making a better system that is effective in detecting cyberattacks in various networks. A Conditional GAN (CGAN) [28], a variant of the model can be used to provide further control over the generated data, such as forcing the model to learn a distribution of a dataset conditioned on its class labels. Part of this dissertation aims to utilize CGAN as an oversampling technique. It is implemented on two network intrusion datasets and evaluated against other oversampling techniques. Furthermore, in this part of the dissertation, we also evaluate the performance of an attempt at combining algorithm-level and data-level approaches. It is shown that this kind of hybrid approach does help improve the intrusion detection performance after its impact is evaluated and compared against individual algorithm-level or data-level approaches. According to Sommer and Paxon [13], it is crucial to focus on insightful information obtained from IDS. So, we also provide a comprehensive analysis on the comparative evaluation of all the studied classifiers in combination with various resampling techniques on two different ID datasets. It will help future potential research with insightful information regarding resampling techniques in network intrusion detection. All contributions are summarized below: 
- A comparative analysis of different machine learning algorithms and deep learning approaches is conducted to show the impact of various resampling techniques on the original classification results.

- A detailed discussion on the experimental results of oversampling, undersampling, and balance sampling is provided to understand the importance of the resampling techniques. (Chapter $4[\mathrm{~J} 1]$ )

- A customized voting classifier is developed and evaluated its performance with other resampling techniques. (Chapter $4[\mathrm{~J} 1]$ )

- A novel network intrusion detection solution is proposed that merges the idea of combining a data level approach (CGAN) with an algorithm-level approach (AE-RL) to improve the performance of the AE-RL.

- CGAN is used as an effective oversampling technique for the first time in the network intrusion detection domain. (Chapter $5[\mathrm{~J} 2]$ )

- This solution is also compared and analyzed with alternate classifiers (Naïve Bayes (NB), Multi Layer Perceptron (MLP), Random Forest (RF) and Logistic Regression (LR)) when combined with CGAN. (Chapter 5 [J2])

- Different combinations of classifier-oversampling techniques are applied to provide a comparative analysis with the proposed solution. (Chapter 5 [J2])

- Experimental analysis of oversampling methods is conducted to provide a robust solution to the problem.

- We discuss an empirical study motivating the choice of CGAN over other GAN variant. (Chapter 6 [J3])

- We implement the combination of AE-RL with CGAN on two different network datasets. After evaluating all experiments on these two datasets, the results 
show that CGAN is not an ideal choice for a dataset with extremely low minority samples in its training data. (Chapter 6 [J3])

- We propose a refined AE-RL data selection technique to combine AE-RL with various oversampling techniques on a dataset with extremely low minority number of samples in its training data. (Chapter 6 [J3])

- We provide a detailed analysis of the impact of selecting the number of synthetic samples on different machine learning algorithms. A comparative study is done on the performance of the different machine learning algorithms. (Chapter 6 $[\mathrm{J} 3])$

- In the end, we suggest the choice of oversampling technique on each specific minority class especially when there is no unique distribution on these minority classes. (Chapter 6 [J3]) 


\subsubsection{List of Publications}

The work in this dissertation has resulted in the following publications.

J1 R. Ahsan, W. Shi, J.P. Corriveau, "Network Intrusion Detection Using Machine Learning Approaches: Addressing Data Imbalance", IET Cyber-Physical Systems: Theory \& Applications, 2021 (Accepted)

J2 R. Ahsan, W. Shi, X. Ma, W. L. Croft, "GAN-based Oversampling for Anomaly Detection". Submitted to the journal of IET Cyber-Physical Systems: Theory \& Applications, 2021.

J3 R. Ahsan, W. Shi, X. Ma, W. L. Croft, "Experimental Analysis of Oversampling Methods to Provide Robust Solution to Class Imbalance Problem in Network Intrusion Detection", ready for submission.

\subsection{Outline}

The remainder of the thesis is organized as follows: In chapter 2, the theory of different machine learning algorithms and resampling techniques, the datasets we used, and the evaluation metrics that we considered are described. In chapter 3, we summarize the literature review in three parts: related work in standard machine learning algorithm, deep learning approaches, and handling data imbalance in the network intrusion detection system. In Chapter 4, we provide a comparative evaluation of ten classifiers combined with three different resampling techniques. This chapter includes the work of [J1]. In Chapter 5, we focus the impact of oversampling techniques on IDS. This work contains the research work reported in [J2]. Chapter 6 further extends our research study by providing a more robust solution and in-depth analysis of the usage of the oversampling technique. This research work is in preparation to be submitted to IEEE Transactions on Big Data [J3]. Beyond the conclusions made 
on each individual study reported in Chapter 4, 5 and 6, we make further conclusions and point out future research directions in Chapter 7. 


\section{Chapter 2}

\section{Background}

\subsection{Supervised Machine Learning Algorithms}

The goal of supervised machine learning is to develop a mapping between input and output spaces, and the training set consists of pairs of input and desired output [29]. Once the algorithm successfully finds the pattern between the input and output, it can easily predict the output for an unseen record. It is mainly used for classification, and regression problems [30]. The most important thing in supervised learning is model complexity and bias-variance trade off. By meaning model complexity, it refers to the ambiguity of a machine learning model. Model complexity largely depends on the training data. If there are not enough training data, it is better to use a less complex model. Because a complicated model may overfit the training data, which results in poor testing results. Overfitting refers to the scenario where it learns the training data so well that it cannot generalize to other records that are not present in the training data. Model generalization is a very important thing for bias-variance trade off. There is always a bias-variance trade off. Here bias is the constant error term, and variance is the stability of the model. If a model is stable, it will give similar results in every run. Contrary, if a model is very biased, it will overfit the data, resulting in bad results. The variance will decrease when bias is increasing and 
vice versa. It is imperative to find a perfect balance in this bias-variance trade off while doing classification tasks with supervised machine learning algorithm.

In this dissertation, a total of 9 different supervised machine learning algorithms, including deep learning algorithms, have been used. A brief description of all the classifiers with their pros and cons is given below.

\subsubsection{Decision Tree}

One of the most widely used classifiers is decision tree [31]. It is a logic-based algorithm. A decision tree can create a logical decision path based on the features along with their labels. When new data comes, it gives a prediction based on the decision path it follows. The decision path is being created by splitting the most significant features into two or more halves. The techniques used to find the most significant features to split into are the Gini index, information gain, etc. [32]. The decision tree's advantage is it is speedy and work very well with the small dataset. This algorithm's main disadvantage is it is prone to overfitting, especially when the tree is deep. It also sometimes doesn’t work well with the large dataset.

\subsubsection{Gaussian Naïve Bayes}

Naïve Bayes mainly provides predictions based on statistical-based calculations [32]. It assumes all the features are independent based on this assumption; it calculates each class's probabilistic density estimation. Based on the highest probabilistic density estimation, it classifies each test instance. And in Gaussian Naïve Bayes, it assumes the data distribution is gaussian. It doesn't perform well when the dataset has a different distribution than the Gaussian distribution. It performs well when the prior probability distribution of a dataset is known. 


\subsubsection{Support Vector Machine}

SVM tries to find the best-suited hyperplane, separating the class points from each other with the maximum margin. When there is a strong margin of differentiation between groups, SVM works well. SVM becomes computationally very expensive when there are so many features. Also, it isn't easy to interpret for the high-dimensional data set, unlike decision tree.

\subsubsection{Random Forest}

Random forest is one kind of hybrid version of the decision trees. Different types of features are used to train different decision trees. And the result is given based on the majority vote. This classifier's main advantage is it is versatile and can be used for both classification and regression. The main problem is it is complicated to interpret the random forest decisions. Also, it is computationally expensive than decision trees.

\subsubsection{Extra Tree}

Similar to Random Forest, extra tree is also an ensemble of decision trees. One of the main differences between extra tree and random forest is extra tree randomly selects the split point to sample the features. In contrast, random forest uses a greedy algorithm to find the ideal split point. This random splitting makes the decision trees result less correlated, which increases the variance of the algorithm. The advantage of this algorithm is computational efficiency [33].

\subsubsection{Logistic Regression}

Logistic regression is a statistical model that models a dependent variable using a logistic function. It tries to learn the linear relationship in a given dataset then uses sigmoid function to introduce a non-linearity. The advantage of Logistic regression 
is model is easy to interpret and efficient to train. It doesn't need to make any prior assumptions about the distributions of class features. But it assumes linearity between the dependent variable and the independent variable, which is a drawback. Logistic regression can be overfitted in a high-dimensional dataset, but it can be avoided if regularization can be added along with the logistic regression. In the scikit learn library [34], two regularizations are available: L1 and L2 regularization. In this dissertation, both of the regularizations have been used to create two different logistic regression models. Regularization is typically used to decrease the generalization error but not the training error [35].

\subsubsection{Neural Networks}

Neural Network is a very popular algorithm nowadays. In this dissertation, multilayer perceptron has been applied. It uses a backpropagation algorithm to train on the training dataset. Then, it updates its weight to produce the results. Neural Network has typically ample amount of hyperparameters. Tune the hyperparameters of the neural network is very crucial to the performance of the neural network. The main advantage of the neural network is it performs very well in a complicated dataset. And the main downfall of this algorithm is it is very prone to overfitting. And it has a very complex architecture.

\subsubsection{Autoencoder}

Autoencoder is constituted by two major functions: an encoder and a decoder. It is a special type of neural network. At first, encoder compressed the data into a reduced dimensionality. Then the decoder decodes it back to the original latent representation. When it decodes, it tries to reconstruct closely to the actual output. There is a reconstruction error, and autoencoder trains to optimize this reconstruction error. 
After training, the encoder has learned about the latent representation of the data. When the encoder is connected to a neural network and trained again, the encoder extracts important information by encoding it into the latent dimension. It helps neural network to learn more efficiently in the latent dimension. It can be used as a dimensional reduction technique, denoising, and as generative modeling. The autoencoders' downfall is that it tries to obtain as much information as possible instead of obtaining relevant information. When the testing dataset has a different representation than the training dataset, autoencoder cannot find relevant information to predict unseen records.

\subsection{Reinforcement Learning}

$\mathrm{RL}$ is the process of figuring out how to map scenarios to actions in order to maximize reward. In the beginning, an intelligent agent that is responsible for the decisionmaking process does not know what is the best action to make; thus, it learns by trial and error simulations in a dynamic environment to obtain the highest reward to make the correct decision. The most interesting thing here is that an action might not be beneficial for the immediate reward, but it will help to gain all other subsequential rewards in the future [36]. Reward can be two types such as positive and negative. For correct decision-making, an agent receives a positive reward, and for an incorrect decision, it receives a negative reward to correct its decision. The agent aims to maximize the positive rewards by making proper decisions. The agent keeps learning through this trial and error process; when the training finishes, the agent can predict the testing dataset's class labels. While learning this process agent faces a trade-off between exploitation and exploration. An agent needs to explore to make better decisions to obtain the maximum rewards in the future. Whereas it also needs to exploit existing knowledge obtained by previous decision making. It is not 
possible to succeed in both tasks without failing others. The rate of exploration and exploitation are referred to as $\epsilon$ and $1-\epsilon$. Initially, exploration remains very high such as 1. And gradually, it decreases over time, whereas exploitation starts at 0 with no prior knowledge. It starts to increase over time when it accumulates knowledge based on its interaction. A trained model mainly uses its exploit knowledge rather than its explored knowledge [37]. There is some advantage of using Reinforcement learning. Firstly, errors in the training period can be fixed later. Then, once the error is solved, there is less chance for the same error. It tries to maintain the balance between exploitation and exploration, whereas other classifiers do not maintain the balance. Finally, it can solve very complex problems that other common techniques cannot solve. There are hardly any disadvantages of reinforcement learning. One notable downfall can be that it requires a lot of data, and it is very computationally expensive.

\subsubsection{Adversarial Environment Reinforcement Learning}

A variant of Reinforcement Learning is Adversarial Environment Reinforcement Learning (AE-RL). When multiple agents functioned in an adversarial environment, it can be called Adversarial Reinforcement Learning. In [25], AE-RL has been used to utilize the RL power in the imbalanced network intrusion detection domain. They used two intelligent agents here. Regular intelligent agent refers to attacker agent, and another agent named environment agent is being introduced here to solve the imbalance problem of the dataset. Attacker agent and environment agent have the adversarial rewards system, which encourages environment agent to select difficult samples and present them to the attacker agent to increase its misclassification. Then, the attacker agent becomes proficient in recognizing difficult attack samples. Environment agent utilize the RL environment's exploration-exploitation mechanism to prioritize minority samples, resulting in a more balanced distribution. It solves the 
exploration problem of the RL algorithm in an imbalanced dataset. Dynamic sampling process of the AE-RL is indifferent to other oversampling or undersampling techniques. In undersampling techniques, majority class samples are being deducted before the training, but AE-RL does sampling dynamically. Also, oversampling techniques generate synthetic samples close to the original dataset, but it is not identical. On the contrary, AE-RL only uses the original samples and retains the original information of the dataset. This is the first work in the network intrusion detection system using AE-RL. It proved to be more efficient as an algorithm-level approach towards data imbalance problems in this domain. It opens a new door of research to utilize this algorithm

\subsection{Resampling Techniques}

In the imbalanced domain, it is always challenging to detect minority classes. It becomes even more difficult in the intrusion detection system, where most of the time, datasets remain highly imbalanced. There are many techniques to solve the imbalance problem of the dataset. Among all the solutions resampling technique is the most used technique. Resampling technique can be divided into three categories: Oversampling Technique, Under Sampling Technique, Balanced Sampling Technique. A brief description of all the sampling techniques is given below.

\subsubsection{Oversampling}

It is a technique where new synthetic samples are created for the minority classes to address the dataset's imbalance problem. When there are enough data for a particular class, it becomes easy for the classifiers to learn about it. It is always beneficial to have enough real data to improve the performance of the machine learning model. But in real life, collecting data for every class is not an easy task. In this scenario, 
oversampling techniques are used to generate synthetic samples that represent the original data distribution. Some of the commonly used oversampling techniques are described below SMOTE is one of the widely used oversampling techniques [38]. SMOTE works by choosing different options closer to the feature space by selecting the nearest neighbor of a minority sample and drawing a new sample from the line between neighbor and minority sample [15]. SMOTE gives all the minority class samples equal weight. In ADASYN, it uses a weighted system for different minority classes as per their difficulty of learning. This means that the samples in the minority class that are harder to learn have more synthetic samples after the oversampling [39]. Apart from these complicated techniques, Random Oversampling is very easy to implement. It just upsamples the minority class samples randomly. It doesn't create any synthetic samples like SMOTE and ADASYN. But It replicates existing samples, which creates bias and reduces the generalization of the machine learning model.

\subsubsection{GAN as an Oversampling Techniuqe}

When training a GAN, the goal is to learn a mapping of noise drawn from a random distribution to an approximation of the desired data distribution. When successfully trained, the model is able to produce highly realistic samples. This ability to produce novel, high quality data is of particular use for the task of data augmentation and has been investigated as a means to balance class distributions in unbalanced datasets by oversampling minority classes [40, 41].

A GAN typically consists of two multi-layered, feed-forward neural networks: a generator and a discriminator. The task of the generator is to learn the mapping from a multi-dimensional latent space to the data distribution. The latent space is often sampled using a normal or uniform distribution. The discriminator is used to scrutinize the quality of the generated data by learning to classify samples as 
being either real or fake (i.e., generated). The output of the discriminator is thus a scalar value representing the probability that the input sample is real. A diagram depicting the overall structure of the model is given in Figure 2.1. Dashed arrows denote the auxiliary information present only in the CGAN variant. In both variants, the generator $G$ accepts as input random noise $z$ sampled from a distribution $p_{z}$ and produces as output fake data $\tilde{x}$ from an approximation of the training data distribution $p_{\text {data }}$. The discriminator $D$ accepts as input fake data $\tilde{x}$ or real data $x$ sampled from $p_{\text {data }}$ and outputs a value in the range $[0,1]$. In the CGAN variant, both networks additionally accept as input auxiliary information $y$ sampled from $p_{\text {data }}$.

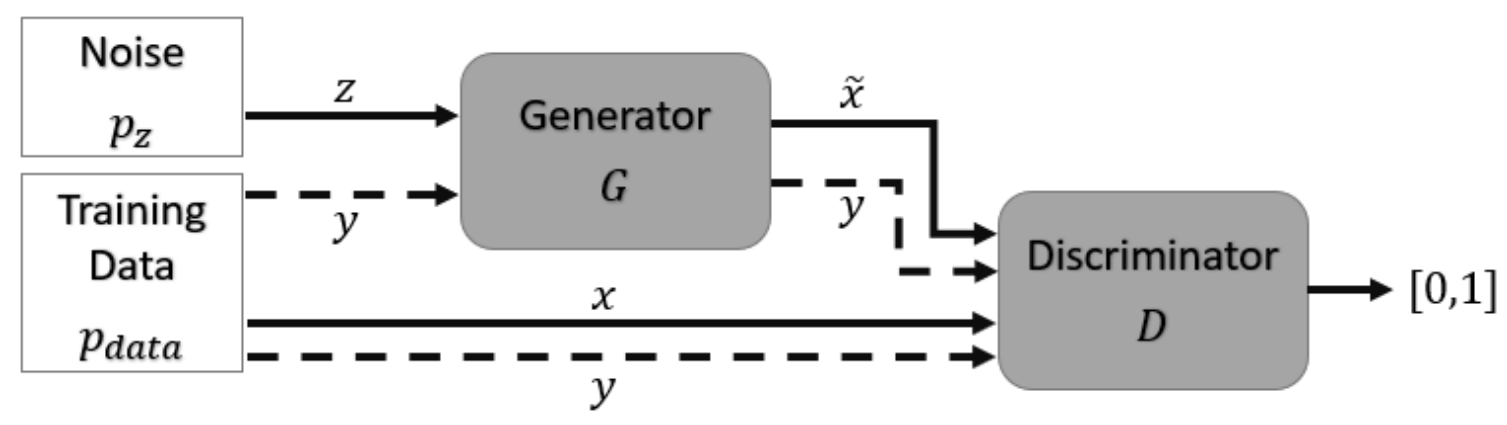

Figure 2.1: Flow of data through the GAN model

The two networks compete in a minimax game during training. The discriminator aims to correctly distinguish between real training samples and fake samples while the generator aims to produce high quality samples capable of fooling the discriminator. The adversarial nature of the training forces both networks to continually improve in order to thwart the other. The networks alternate in updating their parameters during training in order to ensure that neither improves too rapidly compared to the other, as this would lead to training instability. Both networks share the same objective function:

$$
\min _{G} \max _{D} \mathbb{E}_{x \sim p_{\text {data }}(x)}[\log (D(x))]+\mathbb{E}_{z \sim p_{z}(z)}[\log (1-D(G(z)))],
$$

where $G$ and $D$ are the generator and discriminator networks, respectively, $p_{\text {data }}$ 
is the training data distribution and $p_{z}$ is the distribution of random noise used as input for $G$. The generator aims to minimize this value while the discriminator aims to maximize it.

A CGAN [28] variant of the model can also be employed when the goal is to generate samples from a conditional data distribution. This can be used to provide more control over the generated data. For instance, by training the model to learn a distribution conditioned on class labels, it becomes possible to specify the class of samples to be generated by the trained model. In the context of oversampling for the purpose of data balancing, this allows for labels of minority classes to be specified during data generation in order to selectively augment the classes in need of additional samples. At the implementation level, the generator and discriminator networks are modified to accept an additional input that captures the auxiliary information (e.g., a class label). The flow of the auxiliary information is shown by the dashed arrows in Figure 2.1. The updated objective function is given as follows:

$$
\min _{G} \max _{D} \mathbb{E}_{x, y \sim p_{\text {data }}(x, y)}[\log (D(x, y))]+\mathbb{E}_{z \sim p_{z}(z), y \sim p_{y}(y)}[\log (1-D(G(z, y), y))]
$$

where $p_{\text {data }}$ is now a conditional distribution and $p_{y}$ is the distribution of the auxiliary information.

\subsubsection{Undersampling}

A balanced distribution of the majority and minority class samples helps classifiers to perform well. In this technique, samples of the majority are discarded to maintain a balanced distribution of majority and minority classes. Some of the commonly used undersampling techniques are described below: In NearMiss technique, samples of the majority class that are close to other minority class samples are deleted from the dataset [42]. Users can specify how many majority samples they want to delete. Then, Nearest Neighbor strategy is being utilized in ENN algorithm [43]. In this 
algorithm, all the majority instances are being observed along with their nearest neighbor. If $\mathrm{k}$ (the user can specify $\mathrm{k}$ ) nearest neighbors disagree with the current class label of that instance, then it has been discarded. In this way, it edits out noisy instances which are closer to the decision boundaries. Another simple undersampling technique is random undersampling, where samples of the majority class are being dropped randomly.

\subsubsection{Balance sampling}

When oversampling technique is being combined with undersampling technique, it becomes balance sampling. Typically, in balance sampling, oversampling is applied only to the minority classes. And undersampling is applied only to the majority class or to all the classes. In scikit learn library, two available balanced sampling techniques are SMOTEENN and SMOTE-Tomek. Both of them are the hybrid combination of SMOTE with ENN and Tomek, respectively.

\subsection{Selected Network Intrusion Datasets}

\subsubsection{AWID}

AWID [44] is a well-known publicly available dataset. This dataset has been created based on real-world network traffic with a fine mixture of normal activity and anomalous activities. Among the available variants of the dataset, we have selected AWID-CLS-R. Here, the attack types are classified into three major categories (Flooding, Injection and Impersonation), with normal activity as a fourth category. The dataset contains 1795574 training samples and 575642 testing samples. It has in total 154 features (continuous and categorical ones). This dataset is extremely imbalanced, having 1633189 normal instances with only 162385 instances across all attack classes. 
In Figure 2.2, the class distribution of the AWID dataset is shown. The minority classes are similarly sized with each other in regards to their number of samples.

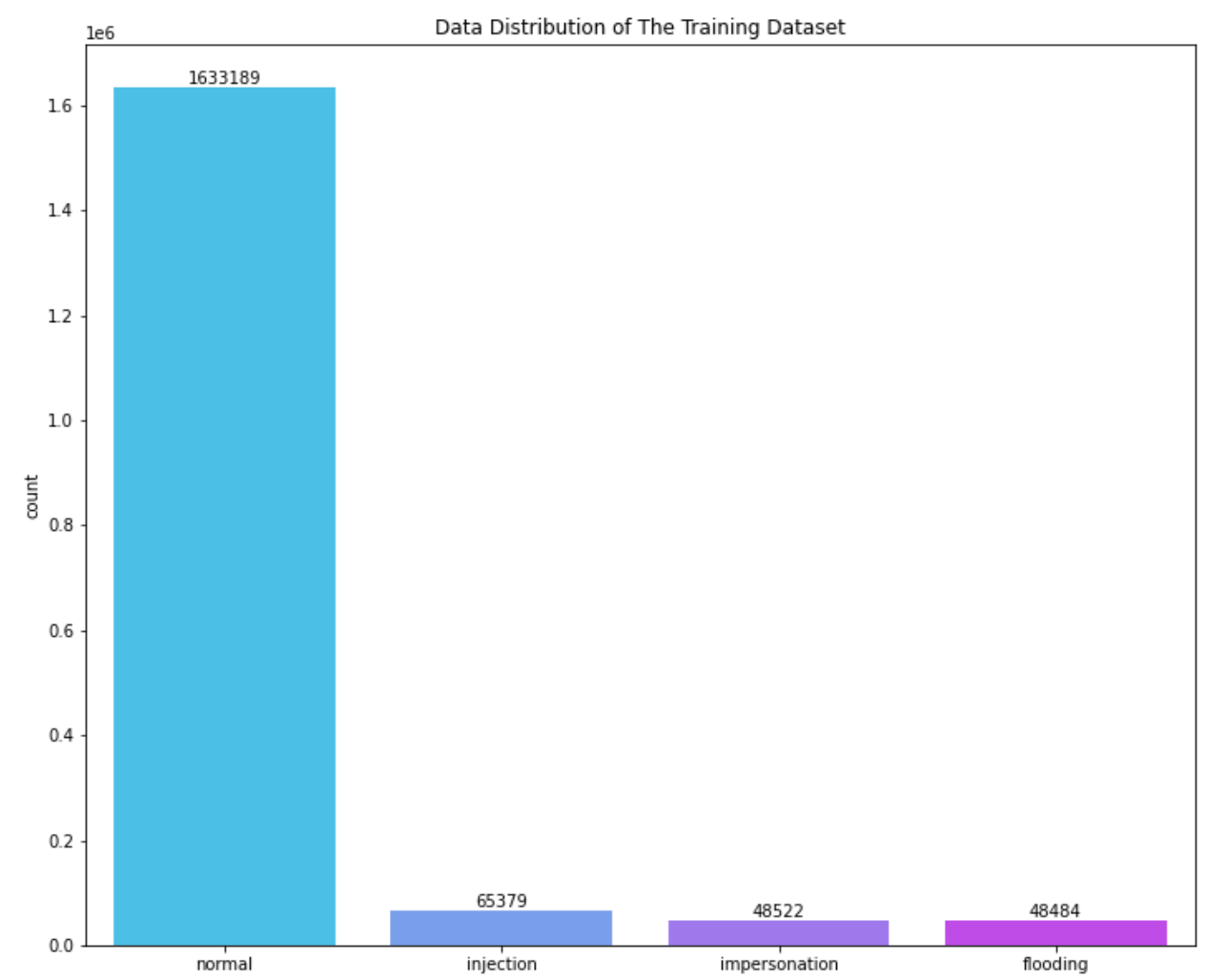

Figure 2.2: Class distribution of AWID training data

\subsubsection{NSL-KDD}

The NSL-KDD dataset [45] originated from KDD-Cup99. It is seen as a refined version of KDD-Cup99. NSL-KDD has predefined training and testing partitions. The training partition is referred to as KDD-Train + , and the testing dataset is referred to as KDD-Test+. In figure 2.3, the training data distribution of NSL-KDD is shown. 
The first sub-figure shows the data distribution over various cyberattacks. The training dataset has 67,343 normal instances out of 125,973 total records. The second sub-figure shows the data distribution over four class into which the cyberattacks are categorized, along with a fifth class for normal instances. These four cyberattack categories are DoS, Probe, R2L, U2R. Even after the categorization, the dataset is still highly imbalanced. In the training dataset, U2R and R2L have only 52 and 995 instances respectively. Related works $[46,47,48,49,25,37,50,51,52]$ show that most multi-class classification studies have been conducted on these five categories.
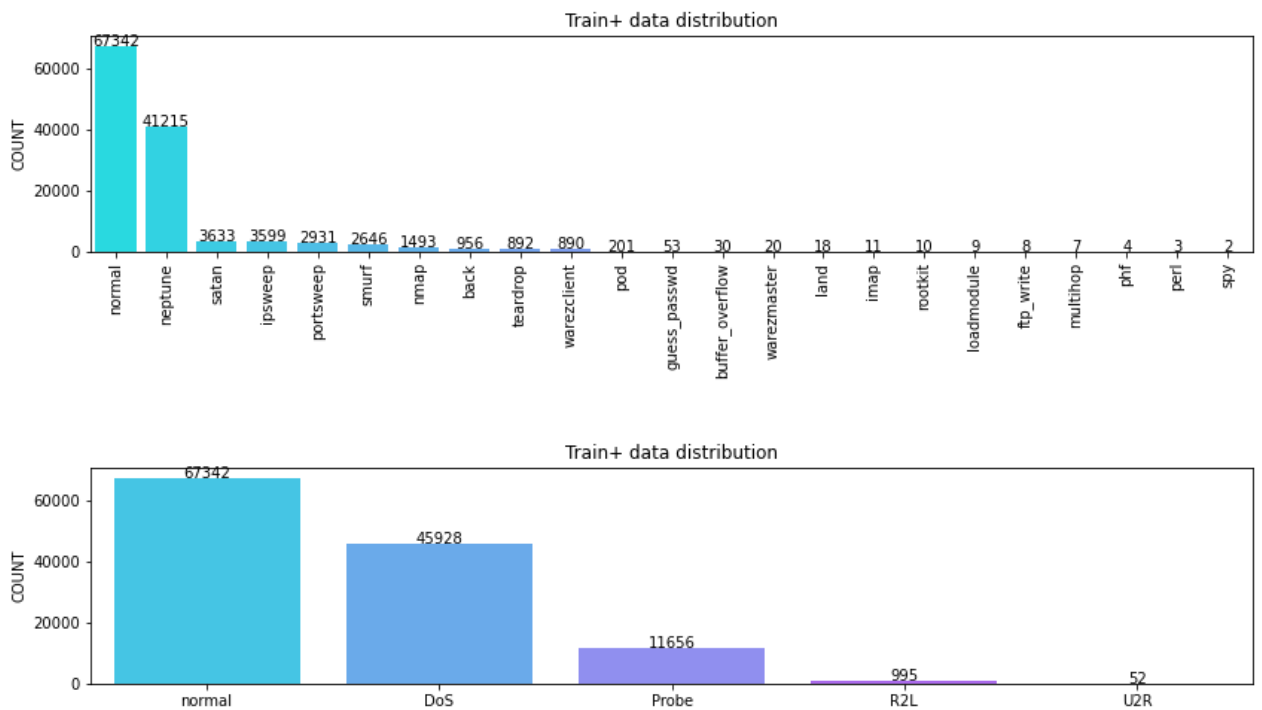

Figure 2.3: Training data distribution of the NSL-KDD dataset.

\subsection{Evaluation Metrics}

Intrusion models are often evaluated using 4 common evaluation metrics: Accuracy , Precision, F1-score and Recall [37]. These evaluation metrics offer a complete view of how different machine learning algorithms perform. Evaluation based solely on the 
accuracy results is not recommended due to the imbalanced nature of the investigated datasets. For the evaluation of machine learning classifiers, we have focused on F1score because of its trade-off between recall and precision. We used the weighted form of F1-score due to the imbalanced nature of the datasets. Weighted F1-score gives priority based on the number of samples of each class presented in the testing data. A good F1-score requires a reasonable detection rate and low false alarm rates [28]. The formula of the F1-score is given in 2.1:

$$
F 1-\text { score }=\frac{2 *(\text { Precision } * \text { Recall })}{\text { Precision }+ \text { Recall }}
$$

However, we have also calculated the accuracy, precision, and recall for all the models. We also investigated the accuracy of the models after the F1-score as an evaluation metric. Accuracy is the ratio of the number of correct predictions against the total number of samples. The formulae for accuracy, precision and recall are shown in eq $2.2,2.3$, and 2.4:

$$
\begin{gathered}
\text { Accuracy }=\frac{\text { TruePositive }+ \text { TrueNegative }}{\text { TotalNumberoftestsamples }} \\
\text { Precision }=\frac{\text { TruePositive }}{\text { TruePositive }+ \text { FalsePositive }} \\
\text { Recall }=\frac{\text { TruePositive }}{\text { TruePositive }+ \text { FalseNegative }}
\end{gathered}
$$

True positive refers to the correctly predicted labels of the positive class. False positive refers to the labels that were predicted as positive but are negative originally, and False Negative refers to the positive labels but falsely predicted as negative. 


\section{Chapter 3}

\section{Literature Review}

This chapter contains important research work that has been conducted using network datasets to identify cyberattacks. All the related works are divided into three major categories: Standard Machine Learning Algorithm, Deep Learning Algorithm, and Handling Imbalance Problem in Network Intrusion Detection.

\subsection{Work-Related to Standard Machine Learning Algorithm}

In [53], machine learning algorithms like Random Tree, RF, MLP, J48, NB, Bayesian Network, and Decision Table have been used to test the efficiency of the KDD dataset (which is a popular network dataset). They stated that it is impossible for a machine learning algorithm to detect all the attack types efficiently. Among the used classifiers, they successfully achieved the lowest false negative with decision table, and the overall best accuracy rate, $93.77 \%$, was achieved by RF. Then in another research [54], an attack detection system was proposed specifically for Denial of Service (DoS), which uses nine different supervised and unsupervised machine learning classifiers. Decision tree, NB, RF, and SVM with various kernel forms such as linear, poly, and radial basis function were used from the supervised classifiers group. And only K-means 
classification technique was selected from the unsupervised classifiers group. Four different Distributed Denial of Service (DDoS) attacks were analyzed in this research. The finding of this research indicates that their proposed system could detect attacks with an accuracy of $99.7 \%$.

Some researchers tried to improve intrusion detection by applying feature selection techniques. In [55], a hybrid feature selection containing correlation-based feature selection and information gain was proposed to improve the 5-class classification in the NSL-KDD dataset. Adaptive Boosting (AdaBoost) has been used as a classifier to improve the performance with a minimal number of features. Then, another research group calculated the information gain through eXtreme Gradient Boosting (XGBoost) and selected 23 important features in UNSW-NB 15 dataset to improve the classification performance [56]. In order to perform better with minimal features, four different subsets of attribute sets are tested against some well-known classifiers like J48, OneR, RF, Naïve Bayes, Bagging, Simple Logistic, and Multi-Layer Perceptron on AWID dataset [57]. For a meaningful feature selection, an intelligent system was proposed in a study [58] by combining Simulated Annealing (SA) and SVM. It provided better results than other classifiers like SVM and decision trees on UNSW and UNB datasets. In another research [59], a hybrid version of Principal Component Analysis (PCA) - Firefly was used for feature reduction in an IDS dataset. At first, they use one key coding to transform their dataset (an open Kaggle dataset), then they applied the PCA-Firefly feature reduction technique to create a reduced dataset. Four different classifiers (SVM, NB, RF, XGBoost) are used to evaluate this approach. PCA-Firefly with XGBoost outperformed all other classifiers in terms of sensitivity, specificity, and accuracy.

In [50], the authors combined Artificial Neural Network and a Bayesian net in order to form an ensemble classifier. They tested their results on the KDD cup 99 and NSL-KDD datasets. They considered only binary classification and performed their 
studies only on the training dataset provided (using different partitions). The authors of [51] proposed a framework with ensemble classifiers (C4.5, RF, and Forest PA) and CFS-Ba feature selection. Their proposed frameworks were able to obtain $87.37 \%$ accuracy on KDDTest+. Another interesting voting classifier has been proposed by [52]. The authors built an adaptive voting system where they combined a Deep Neural Network (DNN) with other basic classifiers on selected features, which enabled them to obtain $85.2 \%$ Accuracy. This study applied 10-fold cross-validation on the training dataset to calculate the voting classifiers' weight which was then used for prediction.

\subsection{Work-Related to Deep Learning Algorithm}

Deep learning method shows a promising result for cyberattack detection. A deep learning approach was proposed by [60] for the IEEE 802.11 wireless network anomaly detection. Their proposed model was created using Stacked Autoencoder (SAE) with two and three hidden layers, respectively. They conducted a multi-class classification and successful in achieving an overall accuracy of 98.66688\%. In [46], the authors developed a DNN by considering neural network performances against intrusion detection systems. They showed a detailed evaluation of the DNN with various kinds of hidden layers on benchmark network traffic datasets. Their proposed model performed better than other existing traditional machine learning algorithms. In another study [47], a Recurrent Neural Network (RNN) has been used on the NSL-KDD dataset, and in the multi-class scenario, it attained roughly $81 \%$ accuracy. In order to improve the classification results by using neural networks such as Convolutional Neural Network (CNN), the NSL-KDD dataset has been converted into image format and fed into a CNN model. A slight improvement in classification results were reported in [61]. 
An extensive empirical study had been conducted in the network intrusion detection system using deep learning models by [62]. Deep learning models used in this research are DNN, Long Short-Term Memory-Recurrent Neural Network (LSTMRNN), Gated Recurrent Unit-Recurrent Neural Network (GRU-RNN), and Deep Belief Network (DBN). They also used a Particle Swarm Optimization (PSO)-based algorithm to find optimal hyperparameters for the deep learning models in the pretraining phase. They also validated their models against 4 popular Intrusion Detections System datasets. They found out that including pretraining improves the Detection Rate (DR) by $4 \%$ and decreased the false alarm rate from $3 \%$ to $1 \%$. They also used 22 evaluation metrics along with Friedman statistical test and two ranking methods to provide a comparative analysis. Decision forest and decision jungle are selected as shallow learners to compare with their selected deep learning algorithms. All the selected deep learning models were able to outperform the shallow learning algorithms. And among all other deep learning algorithms, DBN performs the best. Additionally, they found that deep learning models in network intrusion detection are capable of increasing the DR from $5 \%$ to $10 \%$ and decreasing False Alarm Rate (FAR) from $5 \%$ to $1 \%$.

It also has been shown that autoencoders perform well as a dimensionality reduction system, and it has outperformed the popular dimensionality reduction method like Principal Component Analysis (PCA) [63]. In [64], an interesting study has been conducted using autoencoder to extract features from the NSL-KDD dataset, which resulted in better False Positive Rate (FPR) and Detection Accuracy than using classifiers like KNN and SVM. In [48], a Conditional Variational AutoEncoder (CVAE) was proposed for the network intrusion detection system, which obtained an accuracy of $80 \%$. 


\subsection{Handling Imbalance Problem in Network In- trusion Detection}

In this section, the related works that have in conducted in the fields of IDS are divided into three parts. One is the algorithm level approach, where different kinds of algorithm level research work have been used to handle imbalance problems. Then, the work-related to data-level approach for handling the imbalance problem is also being described. Additionally, the research work related to GAN in the imbalanced domain is also being discussed here.

\subsubsection{A Review of Algorithm Level Approach}

With algorithm-level approaches, the objective is to strengthen a classifier in terms of algorithm architecture to cope with the class imbalance problem. AE-RL is an example in this context [25]. AE-RL has two agents one is an attacker agent, and another is an environment agent. The attacker agent's job is to predict the attack labels that have been provided in the batch. Based on the attacker agent prediction, it receives a reward point. The environment agent's job is to provide the attacker agent with difficult samples to increase the misclassification rate of the attacker agent. And a reward point is also associated with the environment agent's performance. In short, both agents are being trained in an adversarial way. After training, the attacker agent is being used to predict the class labels of the testing dataset. As the attacker agent is being trained in an adversarial way, it can identify all the individual classes correctly. A comparison between AE-RL and other machine learning algorithms with the best oversampling techniques is also shown in [25]. AE-RL outperforms all of them with a $0.7940 \mathrm{~F} 1$-score on the NSL-KDD dataset and $0.9629 \mathrm{~F} 1$-score on the 
Aegean WIFI Intrusion Dataset (AWID). Although it performs well in the imbalanced domain, it still suffers from the scarcity of the cyber attack data [37]. Due to insufficient variational data, the classifier struggles to predict unknown classes. To solve this problem, the authors of [37] proposed another framework named Adversarial Reinforcement Learning with SMOTE (AESMOTE) by combining data level approach SMOTE with an algorithm level approach like AE-RL. This research is only conducted on the NSL-KDD dataset, and the result they obtained was the best obtained among all other state of the art using Reinforcement Learning (RL) models with an F1-score of 0.8243 on the NSL-KDD dataset. Later the research group of AERL published another work using Deep Reinforcement Learning (DRL) on two IDS datasets [65]. They made a conceptual modification of DRL by substituting a sampling function of registered training instructions for the environment. In addition to sampling the training sample, this new pseudo-environment provides incentives based on identification errors discovered during training. This new technique is also being tested along with four relevant DRL models such as Deep Q-Network (DQN), Double Deep Q-Network (DDQN), Actor-Critic (AC), and Policy Gradient (PG) where DDQN algorithm provided the best results. Among the two chosen datasets, NSLKDD had been used as a binary classification problem, and the AWID dataset has been used for multi-class classification. A comparison with other classifiers is also shown against these DRL algorithms, and DDQN performs the best with 0.9394 and 0.9120 F1-score on AWID and NSL-KDD datasets. In the future, they plan to extend their research on multi-agent and adversarial models.

\subsubsection{A Review of Data-Level Approach}

In [66], upsampling and downsampling methods are applied on CIDDS-001 to solve the dataset's imbalance problem. It is being validated with RF, Voting, Variational Autoencoder, and stacking Machine learning classifiers. Their proposed algorithm 
can achieve 99.99\% accuracy. Later another research was conducted on the same CIDDS-001 dataset in [67]. This study used only six machine learning algorithms such as KNN, Gradient Boosting, AdaBoost, Decision Tree, RF, and Linear Discriminant Analysis algorithms. And for solving the imbalance problem of the dataset, Synthetic Minority Oversampling Technique (SMOTE) was used, and the number of synthetic samples was decided to make the minority classes equal to the average data size. Based on their experimental results, their proposed method increases the detection accuracy of seldom observed intrusions.

In [68], deep variational autoencoders are used to generate reasonable new samples to create a balanced dataset. Their practical experimental results stated that a balanced dataset leads to better performance when facing unknown attacks. A novel Difficult Set Sampling Technique (DSSTE) sampling technique is proposed in [69] for IDS. The proposed algorithm uses Edited Nearest Neighbor (ENN) algorithm to distinguish the dataset into two sets: easy set and difficult set. It uses the K-mean algorithm to reduce the number of the majority classes. For the minority classes in the difficult set, it zooms in and out the continuous attributes to create synthetic samples. This is how it does a balanced sampling in the difficult set and combines it with the easy set to create a new balanced training dataset. It had been validated with NSL-KDD and CSE-CIC-IDS2018 dataset using classifiers like RF, SVM, XGBoost, Long and Short-term Memory (LSTM), AlexNet, Mini-VGGNet. It is also being compared with other sampling techniques like Random Under Sampling (RUS), Random Over Sampling (ROS), SMOTE. The experimental results show how the proposed algorithm improves the classification result. Among all the combinations, DSSTE+AlexNet achieved the highest accuracy of 0.8284 for the NSL-KDD dataset. An effective IDS was proposed using Convolutional Neural Network (CNN) in [70]. In order to handle the imbalance issue, SMOTE and Gaussian Mixture Model (GMM) were combined to create SGM, which is a balanced sampling technique. This 
proposed SGM was connected with CNN worked as an IDS for the imbalance dataset. Their proposed model was validated on UNSW-NB15 and CICIDS2017 datasets and achieved superior results against similar research work. In [49], the authors discussed various types of oversampling techniques like SMOTE, Adaptive Synthetic Sampling (ADASYN), etc. performance on the NSL-KDD dataset. Their proposed Generative Variational Autoencoder (GVA) oversampling technique was proven to be a better oversampling technique as compared to other oversampling methods with classifiers like RF, Linear SVM, Logistic Regression, and MLP. In that study, they obtained the best result using MLP, with an accuracy of $79.26 \%$ and a F1-score of $76.45 \%$. The authors of [71] worked on explicitly different resampling techniques on network intrusion datasets like KDD99, UNSW-NB15, UNSW-NB17, UNSW-NB18. From the experimental results, they found that if a dataset is highly imbalanced, both oversampling and undersampling technique increases recall sharply. And dataset which is not highly imbalanced resampling techniques has hardly any impact. Also, the oversampling technique it is focusing more on detecting minority classes in general.

\subsubsection{Usage of GAN in The Imbalanced Domain}

GAN framework is a popular machine learning approach for creating synthetic data. Oftentimes, researches applied the GAN framework in the context of producing synthetic samples for cyberattack datasets [72]. GAN has a disadvantage owing to mode collapse and unstable training and lacks the consideration of the majority class samples that affect the classification boundary [73]. In [74], the authors proposed an Outlier Detectable-GAN (ODGAN), which uses a discriminator as an outlier detector to quantify the difference between the distributions of the majority and minority classes. In [75], the authors proposed a novel Single-Objective Generative Adversarial Active Learning (SO-GAAL) method to generate potential outliers for data in highdimensional space as these outliers may provide information to assist the classifier in 
describing a boundary that can separate outliers from normal data effectively.

In [40], the authors proposed a solution for making classification on credit card fraud datasets, which are strongly imbalanced. It adopts the GAN framework to output synthetic minority class samples and then merges them with the original training set to form an augmented set. Experiments show a significant improvement in a classifier's performance trained on the augmented set over that of the same classifier trained on the original data. In [41], the authors proposed an alternative approach that uses the conditional version of the GAN (CGAN) to generate data for the minority classes. Authors in [76] proposed an Imbalanced Generative Adversarial Network-based Intrusion Detection System (IGAN-IDS), which introduces an imbalanced data filter and convolutional layers to the typical architecture. The framework utilizes a data filter, which restricts the GAN training data to only the minority classes. The work in [77] introduces Autoencoder-Conditional GAN (AE-CGAN), a novel framework that processes data characteristics to a lower level by using an autoencoder prior to GAN oversampling. The use of a Conditional Wasserstein GAN with Gradient Penalty (CWGAN-GP) for class balancing is proposed in [73]. The Wasserstein objective function provides greater stability during training, mitigating issues such as mode collapse that are common in standard GAN models. In [78], the authors proposed SAVAER-DNN that can both detect minority class samples and unknown attacks. The framework consists of a combination of a supervised variational auto-encoder with regularization and Wasserstein GAN with Gradient Penalty (WGAN-GP). It uses the encoder-decoder process to synthesize minority class samples and unknown attacks for the purpose of balancing training data. 


\section{Chapter 4}

\section{Comparative Evaluation on Ten Classifiers combined with 3 Resampling Techniques}

\subsection{Methodology}

In this study, various supervised machine learning algorithms have been tested with different pre-processing steps on a benchmark network dataset named NSL-KDD [45]. A comparative evaluation has been conducted on the effectiveness of various machine learning algorithms and along with deep learning approaches. Furthermore, a hybrid voting classifier is used to further improve the results. The task is posed as a multi-classification problem over 5 different classes. All selected classifiers have been tested with different types of resampling techniques. SMOTE is selected from the oversampling family. Among the undersampling techniques, NearMiss has been chosen. Finally, from the balance sampling techniques, a combination of SMOTE and ENN named SMOTEENN is considered in this study. The main workflow of this study is shown in figure 4.1. Firstly, data from the training dataset is pre-processed and fed into the classifiers. After the classifiers are trained, test dataset is used to determine the performance of the classifiers. A total of 4 types of pre-processed data have been used to train the machine learning algorithms: raw data, oversampled data, 
undersampled data, and balance sampled data. A final comparison between all the experimental results is provided in the Results and Analysis section.

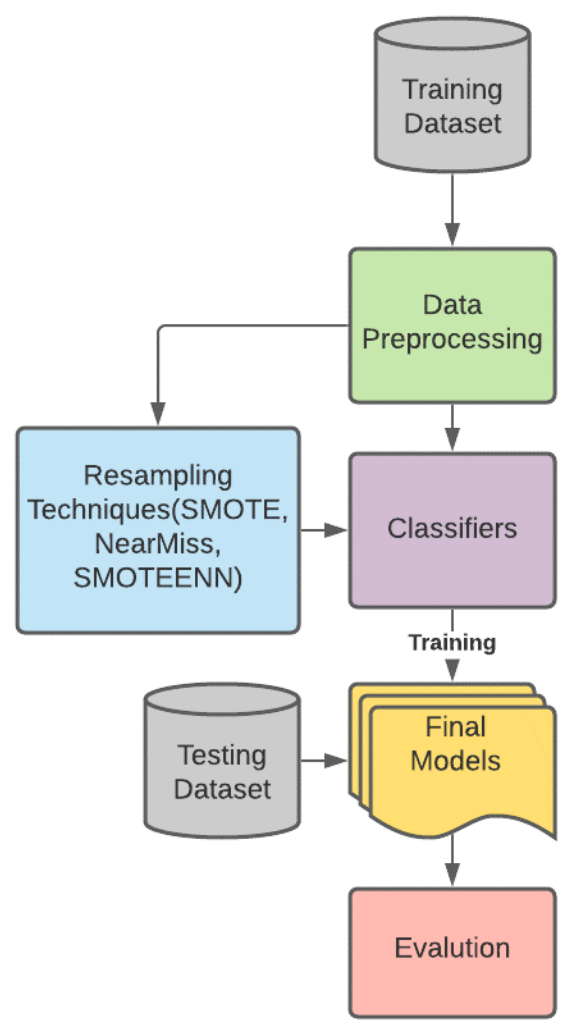

Figure 4.1: Workflow.

\subsection{Data Pre-processing}

The pre-processing step is an essential task in knowledge learning [79]. After looking at the mean and standard deviation of all the features, there are considerable differences between the numeric features, which might create biased results while using machine learning algorithms. Therefore, Minmax scaling is applied over the numeric attributes. The formula of the Minmax scaler is given in (4.1). 


$$
X_{\text {MinMax }}=\frac{x-x_{\text {minimum }}}{x_{\text {maximum }}-x_{\text {minimum }}}
$$

After applying Minmax scaling, all the feature values fall in the range of 0 to 1. Since there is no feature selection that performs equally well for all classifiers studied in this paper, we choose not to perform any feature selection in the pre-processing stage of this study. However, in the pre-processing stage, we apply data changes such as data transformation, scaling, and resampling techniques that are equally beneficial for all the classifiers.

Most machine learning algorithms cannot process categorical data features. In the NSL-KDD dataset, there are three categorical features: 'protocol type', 'service', and 'flag.' These features are important for the classification task. Therefore, we apply one-hot encoding to these features as part of the data pre-processing stage. After applying a one-hot encoder over the categorical features, the total number of features become 122 .

Because the dataset is highly imbalanced, we employ three popular resampling techniques (instances of oversampling, undersampling and balance sampling) to evaluate their impact on the final classification results. A detailed comparative evaluation of the resampling techniques along with the raw data collected are discussed and reported in the Results and Discussions section.

SMOTE [15] is chosen as the oversampling technique used in this study. In the training dataset, normal class contains 67342 samples. All other classes are resampled to 67342 instances using SMOTE over-sampling technique. This is done to create a balanced dataset, preventing the normal instances from dominating the classifier's prediction results.

NearMiss [80] is a well-known undersampling technique. For this study, it is applied only to the Normal class. The records are resampled to 45928 instances, the same as the number of samples in DoS class. A popular approach is to downsample all 
the majority classes and reduce the number of records to that of the smallest minority class. But in this case, the minority class U2R has only 52 samples. Downsampling all the classes to 52 instances would cause significant information loss, consequently resulting in inaccurate classification results.

For balance sampling techniques, SMOTEENN [17] has been used. It is the combination of SMOTE and ENN techniques. It first oversamples all minority classes via SMOTE to make an equal class distribution. After that, it downsamples based on the ENN. After applying SMOTEENN, the final class distribution is normal- 67181, DoS-67308, Probe- 67320, R2L-67325, U2R-67342. The next section will discuss all the machine learning classifiers and proposed algorithms against these four datasets : raw data, oversampled data, undersampled data, and balance sampled data.

\subsection{Machine Learning Algorithms}

\subsubsection{Classical Supervised Machine Learning Algorithms}

To compare the performance on the task of intrusion detection, the following machine learning algorithms are selected to test against NSL-KDD dataset: from the tree-based machine learning algorithms: decision tree, random forest, and extra tree are selected, Gaussian Naïve Bayes algorithm is chosen from the probabilistic models; SVM is selected from the distance-based models; and Logistic Regression with L1 and L2 penalty are chosen from the regression models. All these classifiers are implemented using sci-kit learn library [34] with full list of parameters selected. For tree-based models, the default parameters yield the best results. Logistic Regression L1 \& L2 penalty solver $=$ 'Saga' and tol $=0.001$, SVM with the 'rbf' kernel, Naïve Bayes with var_smoothing $=1 e^{-1}$ gave better results than with their default value. 


\subsubsection{Deep Learning Algorithms}

It is known that fully connected deep neural networks and autoencoders produce good results in anomaly detection systems [81].Therefore, a Fully Connected Neural Network (FCN) with and without an autoencoder are both employed in these experiments.

\begin{tabular}{|c|c|c|}
\hline $\begin{array}{l}\text { Layer (type) } \\
===============\end{array}$ & $\begin{array}{l}\text { Output Shape } \\
== \pm========\end{array}$ & $\begin{array}{l}\text { Param \# } \\
=========\end{array}$ \\
\hline input_1 (InputLayer) & {$[($ None, 122)] } & 0 \\
\hline dense (Dense) & (None, 1024) & 125952 \\
\hline dense_1 (Dense) & (None, 512) & 524800 \\
\hline dense_2 (Dense) & (None, 256) & 131328 \\
\hline dense_3 (Dense) & (None, 5) & 1285 \\
\hline
\end{tabular}

Figure 4.2: FCN Architecture.

A FCN Network is built using Keras [82]. The architecture of this FCN network is shown in figure 4.2. A total of three hidden layers are used, which have the following number of nodes: 1024, 512, 256 respectively. The output layer has five nodes with a 'SoftMax' activation function. For the rest of the hidden layers, a 'ReLU' activation function is used. Figure 4.3 shows that initially, the loss of the validation test was unstable. It becomes relatively stable on 200 epochs. The adam optimizer, with the default parameters, is selected for this model. 'Sparsecategoryentropy' is used as the loss function along with accuracy metrics.

The mechanism of Autoencoder with FCN is already described in the Background section. For the parameter setup of this autoencoder with FCN latent dimension of the encoder is selected as 32. Then, one hidden layer is being used in both encoder and decoder networks, where node sizes are set to 256 and 64, respectively. 30 epochs, and a batch size of 256 are used to train the autoencoder. The activation function of the hidden layers are 'ReLU', the adam optimizer is used, and 'MSE' is used as the loss function in the autoencoder model based on a trial-and-error experiment. The autoencoder combined with the FCN network uses the same architecture as FCN 


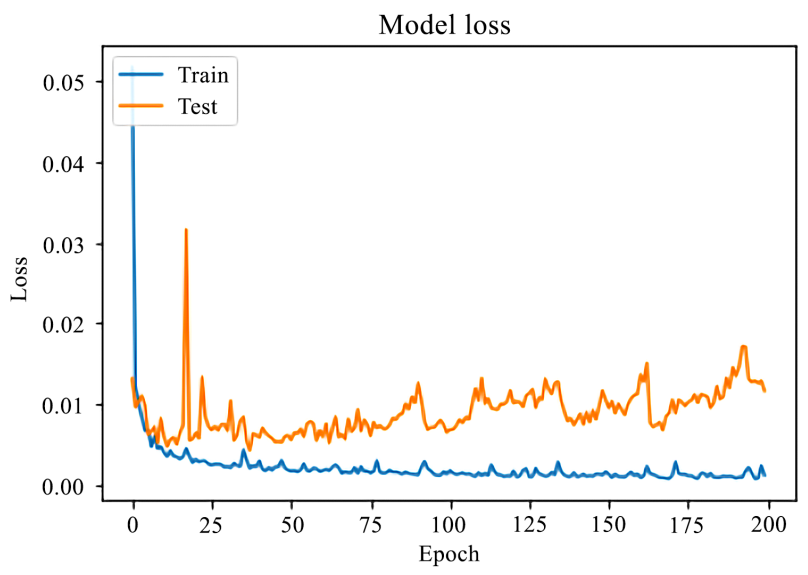

Figure 4.3: Model loss in training and validation datasets in FCN.

architecture mentioned before.

\section{Proposed Voting Classifier}

In [51] and [52], the authors proposed a voting classifier using a set of machine learning algorithms. In this study, a similar type of voting strategy is used on a different set of classifiers. Furthermore, a hard-voting system is used in this study. The proposed voting classifier's architecture is shown in figure 4.4. At first, all the classifiers are trained using either the raw data or resampled data. Then, the trained models predict the class labels of the testing dataset. The final results are based on a majority vote system which decides the final predicted class labels in the testing dataset. In [52], the authors applied a weighted voting system. Furthermore, the authors of [51] did not combine different classifiers like autoencoder with FCN and decision tree together. Even though some classifiers do not produce good prediction results individually, when they are included in the decision-making process of the proposed voting system, the final results are further improved. In our study, the following list of classifiers are included in the voting system: decision tree, naïve bayes, support vector machine, 
random forest, extra tree, fully connected network, autoencoder with a fully connected network as well as logistic regression with $L 1$ and $L 2$ penalties.

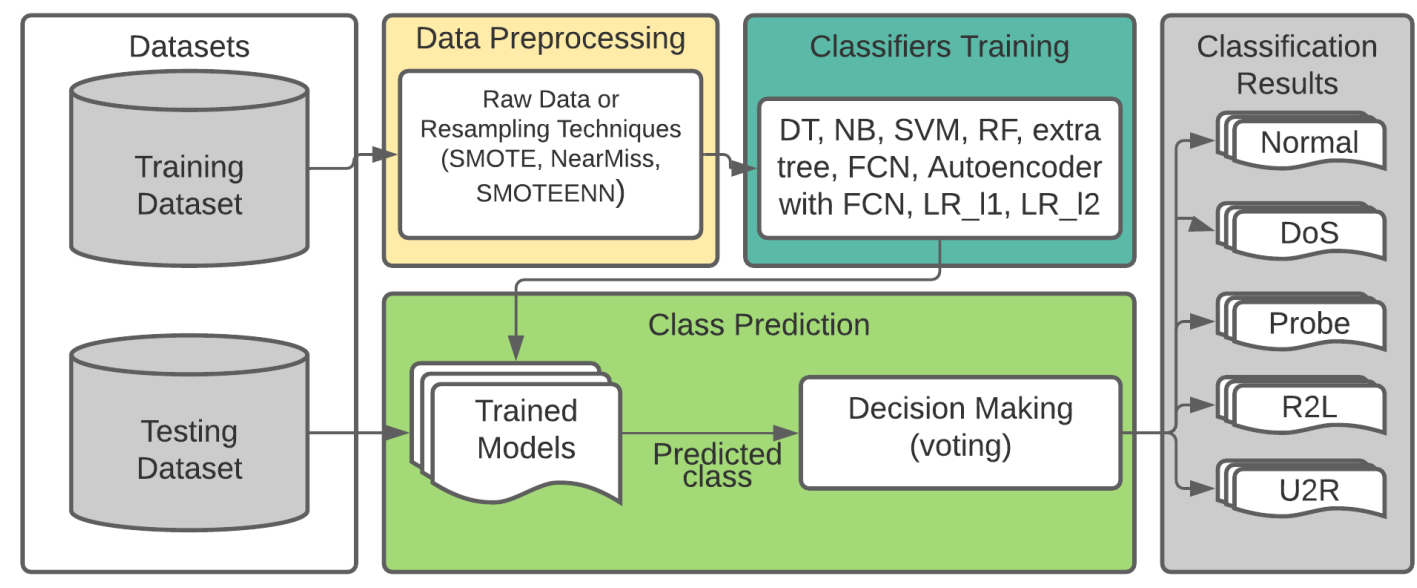

Figure 4.4: Architecture of the voting classifier.

\subsection{Results \& Analysis}

In table 4.1, the results obtained from all nine classifiers as well as the results from the voting classifier are presented. The results show that Naïve Bayes yields the worst performance. Naïve Bayes is a probabilistic model and its predictions are done based on a probability density estimation of a dataset. In the testing dataset, there are new sub-classes that are not present in the training dataset. Therefore, the probability density estimation does not reflect the presence of the new classes, which leads to degraded performance. Almost all the other classifiers produce similar level of results to each other. Logistic regression with L2 penalty demonstrated a slightly better result than the other classical machine learning algorithms. In figure 4.5, the f1-score of all ten classifiers trained on the original dataset are shown. We observe that among all the ten classifiers, the autoencoder with FCN provides the best results. The poor result of the voting classifier obtained from running it on the original dataset follows 
Table 4.1: Comparison of the classifiers on original dataset.

\begin{tabular}{|l|l|l|l|l|}
\hline & Accuracy & Precision & F1-score & Recall \\
\hline Decision Tree & 0.78 & 0.78 & 0.76 & 0.78 \\
\hline Naïve Bayes & 0.66 & 0.75 & 0.65 & 0.66 \\
\hline SVM & 0.79 & 0.82 & 0.77 & 0.79 \\
\hline Random Forest & 0.76 & 0.81 & 0.72 & 0.76 \\
\hline Extra Tree & 0.77 & 0.82 & 0.74 & 0.77 \\
\hline $\begin{array}{l}\text { Logistic Regression } \\
\text { (L1 penalty) }\end{array}$ & 0.78 & 0.80 & 0.75 & 0.78 \\
\hline $\begin{array}{l}\text { Logistic Regression } \\
\text { (L2 penalty) }\end{array}$ & 0.79 & 0.81 & 0.76 & 0.79 \\
\hline $\begin{array}{l}\text { Fully Connected } \\
\text { Neural Networks }\end{array}$ & 0.81 & 0.82 & 0.78 & 0.80 \\
\hline $\begin{array}{l}\text { Autoencoder with } \\
\text { Fully Connected } \\
\text { Neural Networks }\end{array}$ & $\mathbf{0 . 8 2}$ & $\mathbf{0 . 8 3}$ & $\mathbf{0 . 8 0}$ & $\mathbf{0 . 8 2}$ \\
\hline \begin{tabular}{l} 
Voting Classifier \\
\hline
\end{tabular} & 0.79 & 0.81 & 0.76 & 0.79 \\
\hline
\end{tabular}

from the fact that the majority of the individual classifiers we investigated performed poorly due to the imbalance present in the original data.

In table 4.2, the results of combining each of the ten classifiers with SMOTE resampling on the NSL-KDD dataset are shown. In almost all cases, the results are improved compared to using the classifiers without any resampling. Similarly to our explanation before, Naïve Bayes doesn't show any improvement because the model fails to capture the probabilistic density estimation on the new classes in the test dataset. Then, the performance of the autoencoder with FCN network decreased slightly. We believe that this is due to the quality of the synthetic data generated. More precisely, the synthetic data has changed the distribution of the important features, which does not reflect the distribution of the original dataset. Due to this 


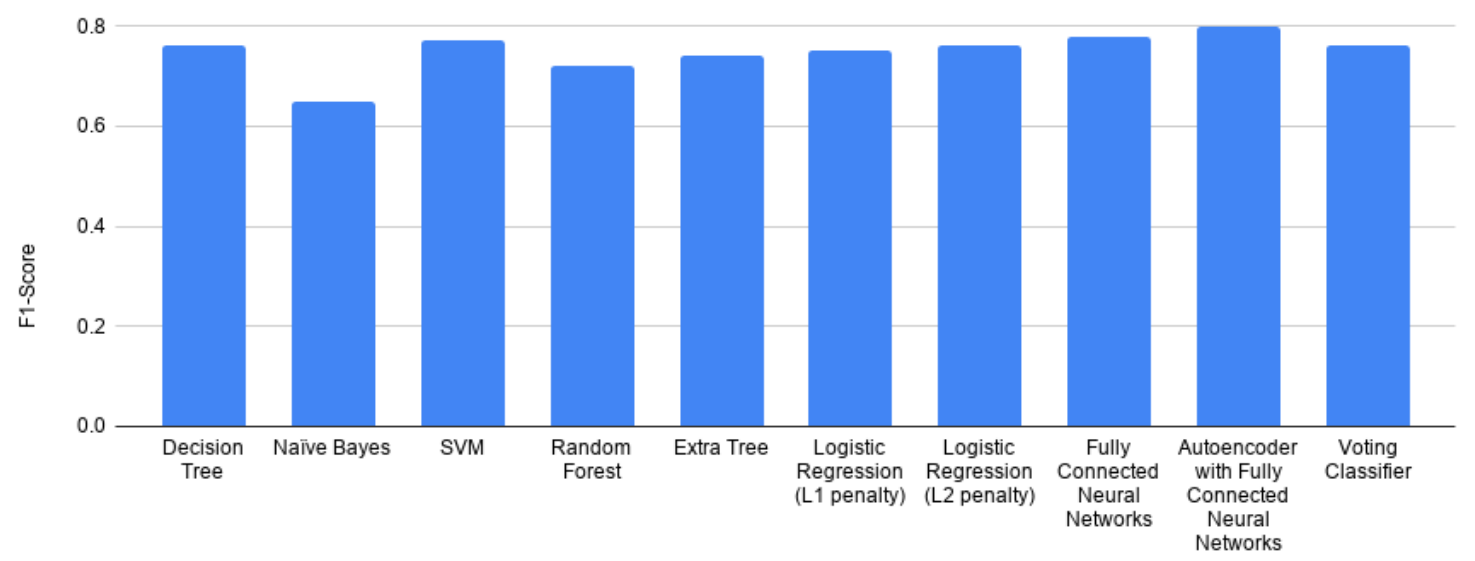

Figure 4.5: F1-scores of the classifiers trained on the original dataset.

change, the autoencoder model fails to capture the distribution of the oversampled dataset. In order to further improve this result, autoencoder structure might need to be changed. As running autoencoder in a large dataset like NSL-KDD is computationally expensive, parameter tuning and architecture changes for autoencoder model are kept as a future work. After applying SMOTE to the dataset, we perform in-

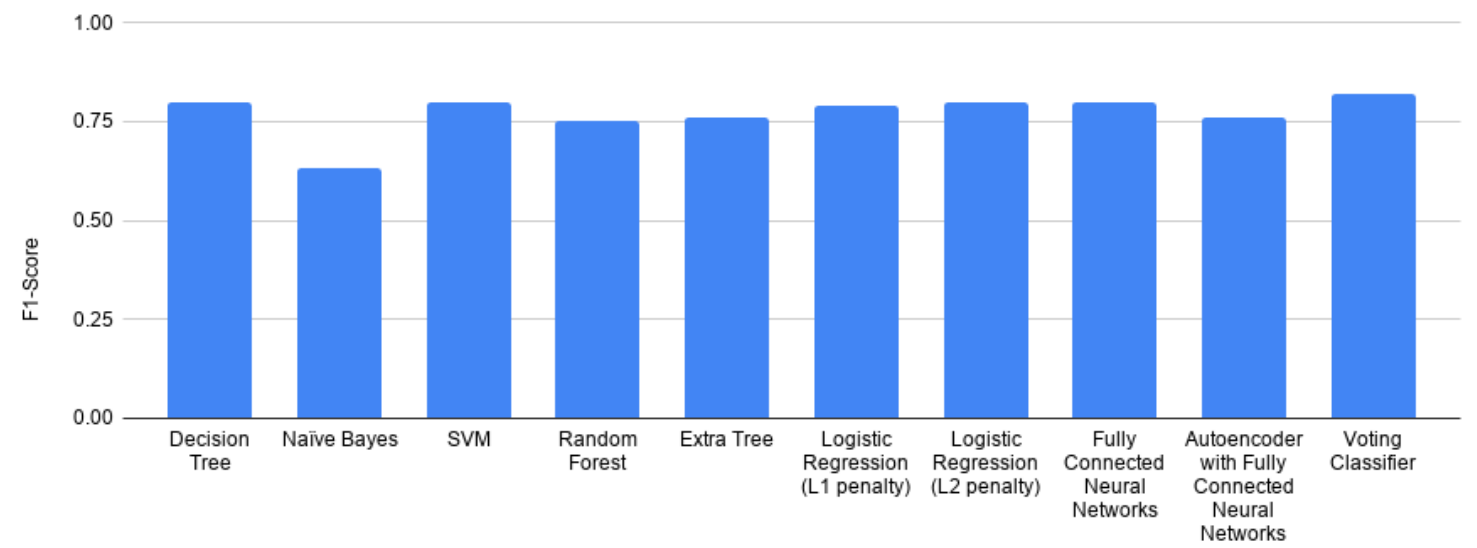

Figure 4.6: F1-scores of the classifiers using SMOTE resampling.

trusion detection using the ten above-mentioned classifiers again on the new training dataset. The proposed voting classifier shows a very good result compared to other classifiers in terms of the F1-score (see figure 4.6). It has 0.83 on accuracy, 0.85 on 
Table 4.2: Comparison of the classifiers using SMOTE resampling.

\begin{tabular}{|l|l|l|l|l|}
\hline & Accuracy & Precision & F1_score & Recall \\
\hline Decision Tree & 0.82 & 0.81 & 0.80 & 0.82 \\
\hline Naïve Bayes & 0.63 & 0.75 & 0.63 & 0.63 \\
\hline SVM & 0.81 & 0.83 & 0.80 & 0.81 \\
\hline Random Forest & 0.78 & 0.82 & 0.75 & 0.78 \\
\hline Extra Tree & 0.79 & 0.83 & 0.76 & 0.79 \\
\hline $\begin{array}{l}\text { Logistic Regression } \\
\text { (L1 penalty) }\end{array}$ & 0.80 & 0.82 & 0.79 & 0.80 \\
\hline $\begin{array}{l}\text { Logistic Regression } \\
\text { (L2 penalty) }\end{array}$ & 0.81 & 0.82 & 0.80 & 0.81 \\
\hline $\begin{array}{l}\text { Fully Connected } \\
\text { Neural Networks }\end{array}$ & 0.82 & 0.82 & 0.80 & 0.82 \\
\hline $\begin{array}{l}\text { Autoencoder with } \\
\text { Fully Connected } \\
\text { Neural Networks }\end{array}$ & 0.79 & 0.80 & 0.76 & 0.78 \\
\hline \begin{tabular}{l} 
Voting Classifier \\
\hline
\end{tabular} & $\mathbf{0 . 8 3}$ & $\mathbf{0 . 8 5}$ & $\mathbf{0 . 8 2}$ & $\mathbf{0 . 8 3}$ \\
\hline
\end{tabular}

precision, 0.83 on recall, and 0.82 on F1-score, which is the highest F1-score achieved so far. The overall performance of the voting classifier is improved significantly because all the classifiers except Naïve Bayes and autoencoder performed better after applying SMOTE resampling.

In table 4.3, we show the results of running all ten classifiers on the test dataset using undersampling technique. The proposed voting classifier and decision tree show the highest accuracy score of 0.82 and F1-score of 0.80 . These results show that all the classifiers perform well with the undersampling techniques compared to the original data.

In figure 4.7, the confusion matrices of decision tree classifier trained on oversampled and undersampled datasets are shown. The True Positive Rate (TPR) of the 
Table 4.3: Comparison of the classifiers using NearMiss Resampling.

\begin{tabular}{|l|l|l|l|l|}
\hline & Accuracy & Precision & F1_score & Recall \\
\hline Decision Tree & $\mathbf{0 . 8 2}$ & 0.82 & $\mathbf{0 . 8 0}$ & $\mathbf{0 . 8 2}$ \\
\hline Naïve Bayes & 0.66 & 0.78 & 0.66 & 0.66 \\
\hline SVM & 0.80 & 0.83 & 0.79 & 0.80 \\
\hline Random Forest & 0.81 & $\mathbf{0 . 8 4}$ & 0.77 & 0.81 \\
\hline Extra Tree & 0.80 & $\mathbf{0 . 8 4}$ & 0.76 & 0.80 \\
\hline $\begin{array}{l}\text { Logistic Regression } \\
\text { (L1 penalty) }\end{array}$ & 0.80 & 0.80 & 0.78 & 0.80 \\
\hline $\begin{array}{l}\text { Logistic Regression } \\
\text { (L2 penalty) }\end{array}$ & 0.80 & 0.81 & 0.78 & 0.80 \\
\hline $\begin{array}{l}\text { Fully Connected } \\
\text { Neural Networks }\end{array}$ & 0.72 & 0.55 & 0.62 & 0.72 \\
\hline $\begin{array}{l}\text { Autoencoder with } \\
\text { Fully Connected } \\
\text { Neural Networks }\end{array}$ & 0.71 & 0.54 & 0.61 & 0.71 \\
\hline \begin{tabular}{l} 
Voting Classifier \\
\hline
\end{tabular} & $\mathbf{0 . 8 2}$ & 0.83 & $\mathbf{0 . 8 0}$ & 0.81 \\
\hline
\end{tabular}

normal class increases from 0.96 to 0.97 and the TPR of DoS, Probe, R2L decreases by $1 \%$. This implies that classical machine learning algorithm like decision tree have a similar type of performance when used with undersampled data compared to the oversampled data.

In figure 4.8, we show the F1-score obtained from training all ten studied classifiers on the dataset after applying NearMiss resampling. All classifiers have very similar F1-score except Naïve Bayes, FCN, and autoencoder with FCN. The FCN and autoencoder with FCN have a low score when combined with NearMiss compared to other resampling techniques. This is beacuase deep learning models need enough data to learn properly and the undersampled dataset offers the fewest samples compared to other resampled datasets. Despite discarding some majority class samples, the 


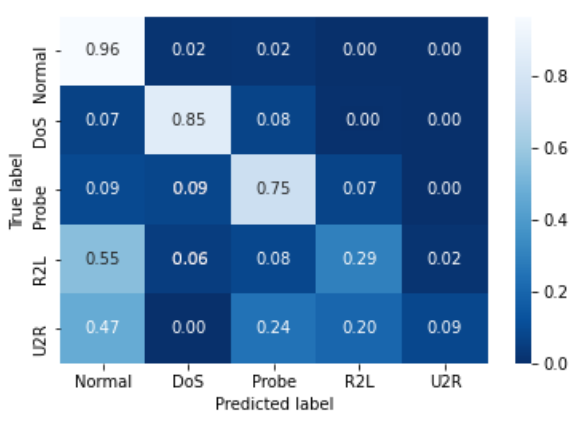

(a) Oversampled Data

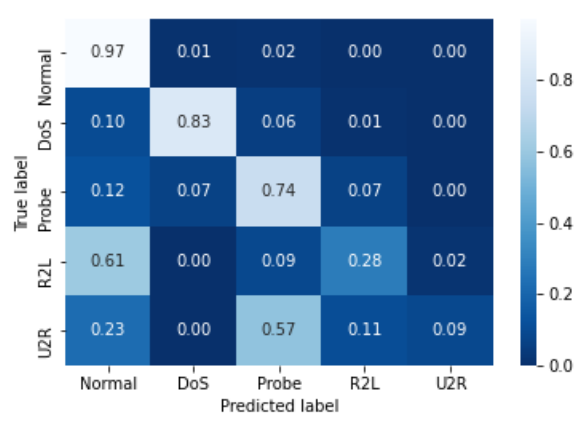

(b) Undersampled Data

Figure 4.7: Confusion matrices of Decision Tree classifier trained on the oversampled dataset and the undersampled training sets.

dataset produced after NearMiss is still highly imbalanced. Furthermore, our neural network architecture has three hidden layers with a fairly large number of nodes which prevent the model loss from stabilizing and overfit the model. This results in incorrect predictions on the minority classes.

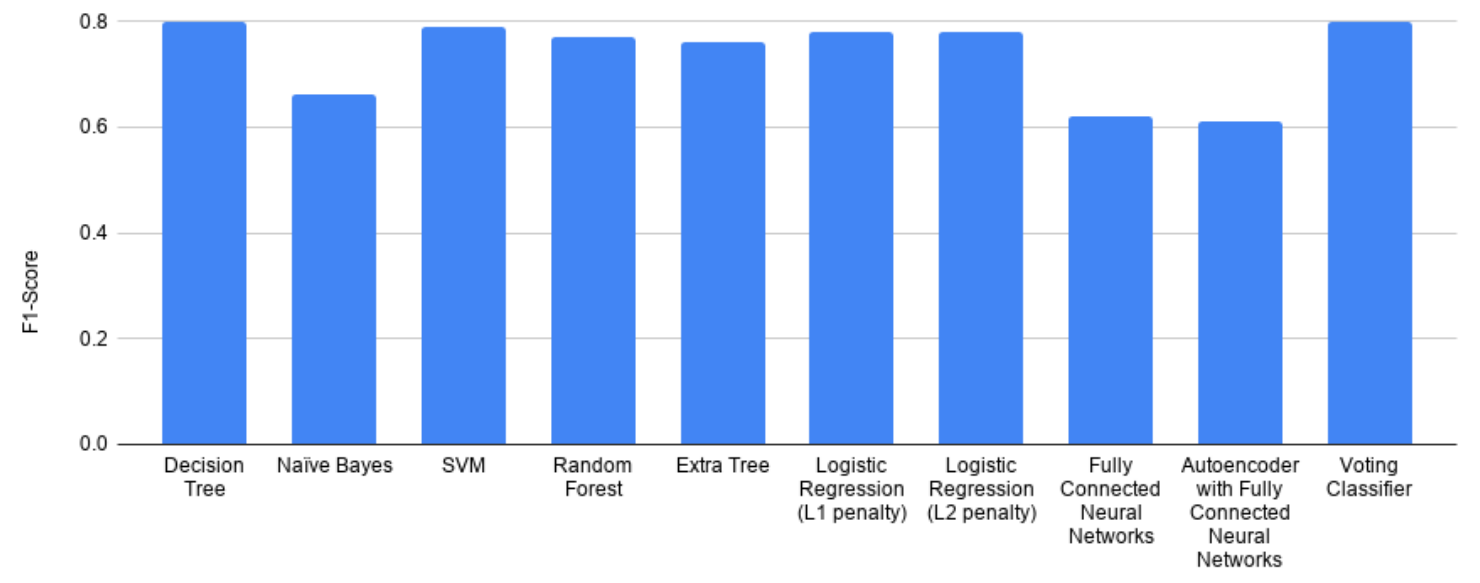

Figure 4.8: F1-scores of the classifiers using NearMiss resampling.

In table 4.4, results obtained from training the classifiers combining with SMOTEENN are shown. In terms of F1-score, SVM performs the best with an F1-score reaching 0.81 . This is the best F1-score that SVM has acquired so far. This is possibly due to SMOTEENN being a hybrid resampling technique combining SMOTE 
Table 4.4: Comparison of the classifiers using SMOTEENN resampling.

\begin{tabular}{|l|l|l|l|l|}
\hline & Accuracy & Precision & F1_score & Recall \\
\hline Decision Tree & $\mathbf{0 . 8 2}$ & 0.82 & 0.80 & $\mathbf{0 . 8 2}$ \\
\hline Naïve Bayes & 0.63 & 0.75 & 0.63 & 0.63 \\
\hline SVM & $\mathbf{0 . 8 2}$ & 0.83 & $\mathbf{0 . 8 1}$ & 0.81 \\
\hline Random Forest & 0.78 & 0.82 & 0.76 & 0.78 \\
\hline Extra Tree & 0.8 & $\mathbf{0 . 8 4}$ & 0.77 & 0.80 \\
\hline $\begin{array}{l}\text { Logistic Regression } \\
\text { (L1 penalty) }\end{array}$ & 0.81 & 0.82 & 0.79 & 0.81 \\
\hline $\begin{array}{l}\text { Logistic Regression } \\
\text { (L2 penalty) }\end{array}$ & 0.81 & 0.83 & 0.80 & 0.81 \\
\hline $\begin{array}{l}\text { Fully Connected } \\
\text { Neural Networks }\end{array}$ & 0.81 & 0.81 & 0.79 & 0.81 \\
\hline $\begin{array}{l}\text { Autoencoder with } \\
\text { Fully Connected } \\
\text { Neural Networks }\end{array}$ & 0.80 & 0.81 & 0.79 & 0.80 \\
\hline \begin{tabular}{l} 
Voting Classifier \\
\hline
\end{tabular} & $\mathbf{0 . 8 2}$ & 0.83 & 0.80 & $\mathbf{0 . 8 2}$ \\
\hline
\end{tabular}

and ENN. These two techniques are based on the distance measure. They successfully created good synthetic samples that are suitable for distance-based classifiers like SVM. We observe that the autoencoder with FCN performs well with SMOTEENN compared to SMOTE. As previously mentioned, the poor results of the autoencoder with SMOTE were caused by the poor quality of the synthetic samples generated by SMOTE. When ENN is added to SMOTE it helps to discard some samples from the oversampled data and keep samples that are related to the original distribution. Therefore, the performance of the Autoencoder with FCN is improved. In figure 4.9, we show the comparison of all ten classifiers trained on a dataset generated from applying SMOTEENN resampling. All the classifiers perform relatively similar in terms of the F1-score. Apart from the FCN and the voting classifier, all other classifiers 
perform a little bit better or remain the same compared to the results when using SMOTE.

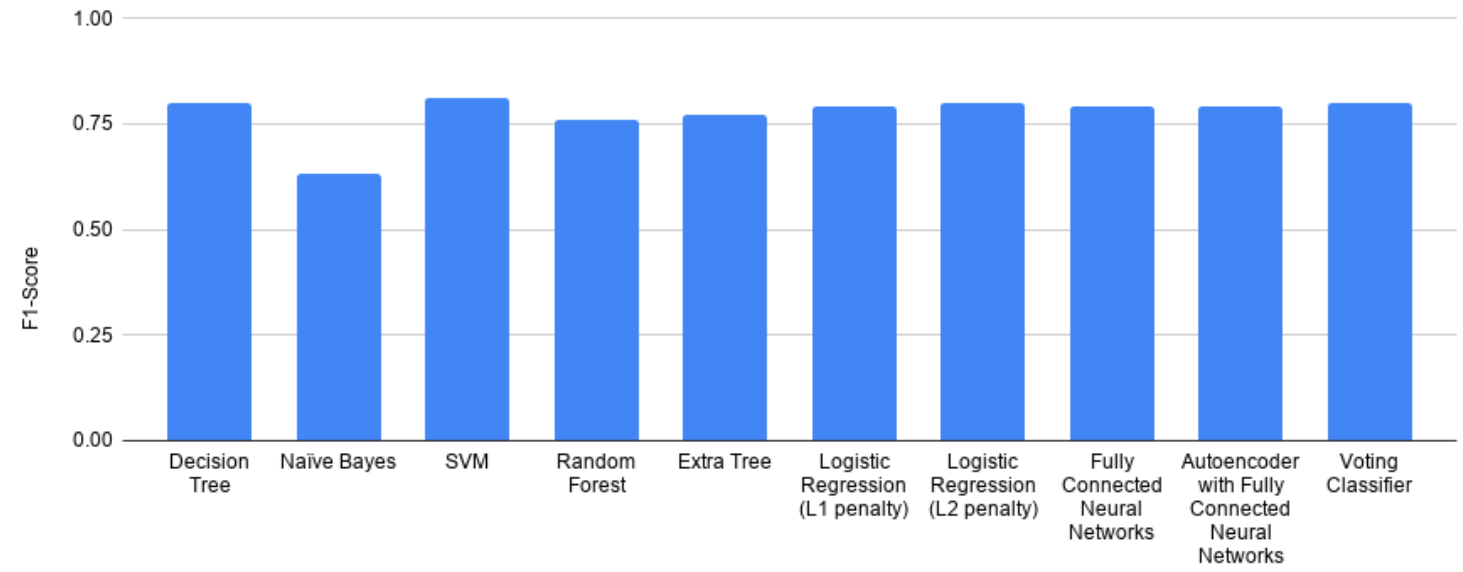

Figure 4.9: F1-scores of the classifiers when using SMOTEENN resampling.

In figure 4.10, we show in a bar chart the differences between the F1-scores of all seven classical machine learning algorithms trained with each of the three resampling techniques as well as the F1-scores from training the seven classifiers on the original dataset. After applying resampling techniques, the F1-scores have increased for all classifiers except Naïve Bayes. This confirms that resampling techniques have a positive impact on the performance of most classical machine learning algorithms.

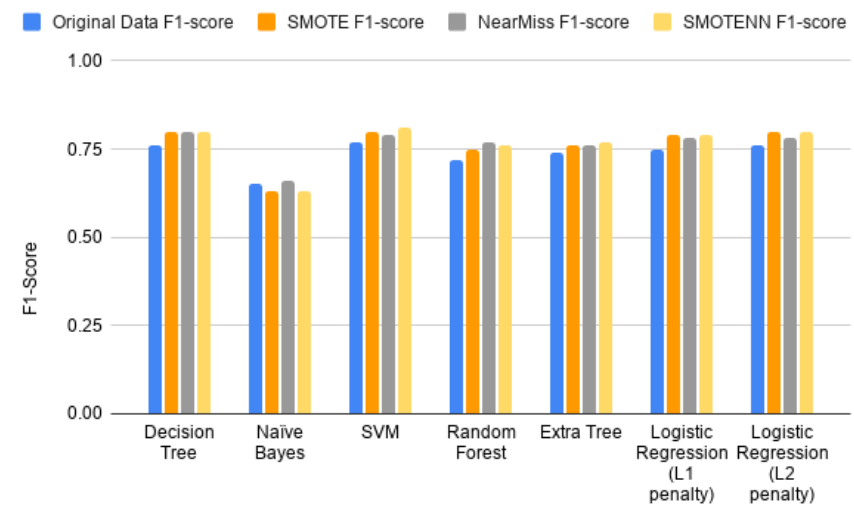

Figure 4.10: F1-scores of the classical machine learning algorithms trained on the original dataset and the datasets generated using 3 resampling techniques. 


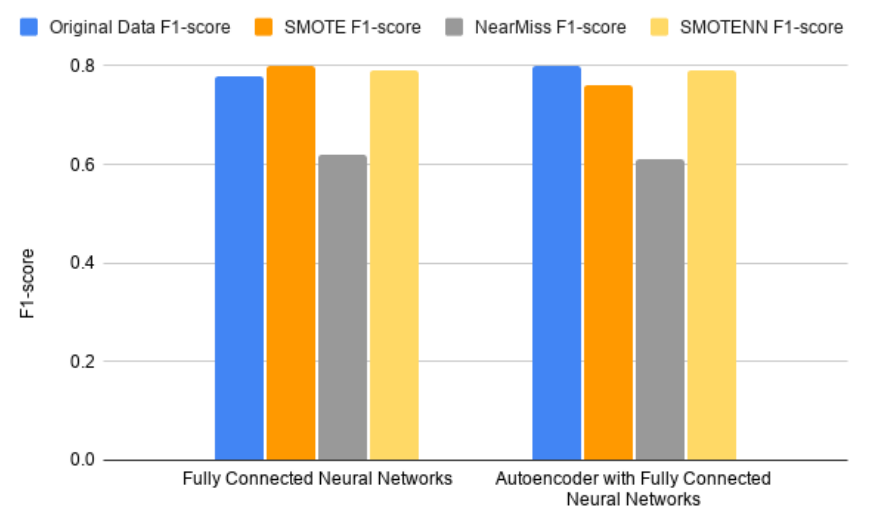

Figure 4.11: F1-scores of the Deep Learning algorithms trained on the original dataset and the datasets generated using 3 resampling techniques.

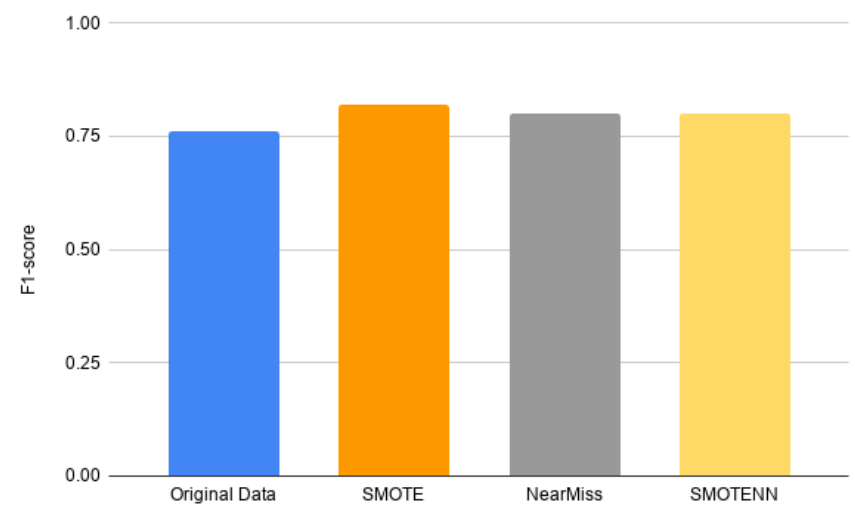

Figure 4.12: F1-scores of the voting classifier trained on the original dataset and the datasets generated using 3 resampling techniques.

In figure 4.11, the F1-scores of the two deep learning algorithms are compared when using the resampling techniques and running on the original dataset. The FCN performs well with SMOTE however, its performance suffers slightly when ENN has been used with SMOTE. On the other hand, the autoencoder cannot capture the original distribution properly with SMOTE and its performance improves when SMOTE is combined with ENN. Deep learning models are more dependent on the training data. Because of the imbalanced distribution of the undersampled dataset and lack of data, our deep learning models do not perform well with undersampling 
compared to other resampling techniques.

We also investigate the impact of these resampling techniques on the performance of our proposed voting classifier. The results compared with those obtained from trained on the original dataset are shown in figure 4.12. We attain the best F1-score for the voting classifier when it is combined with SMOTE. The results confirm that the use of majority voting does improve intrusion detection. Also, the voting classifier performs well with the resampled dataset which implies that resampling techniques provide sufficient variation to all classifiers to improve their classification performance. 


\section{Chapter 5}

\section{Study on the Impact of Oversampling Techniques on IDS}

Three different types of resampling techniques were primarily used in the network intrusion dataset to handle the imbalance problem. From the obtained results, it is evident that creating synthetic samples is much more effective than discarding samples. The next part of the research study is entirely focused on using oversampling technique in the network domain.

\subsection{Problem Statement \& Solution}

Current research on the classification of imbalanced data is mainly summarized into two classes of approaches. The first targets the algorithm-level through the use of classifiers designed with imbalanced data in mind. The objective is to enable the classifier to adapt or strengthen its learning process for the minority classes. The second approach involves data-level modifications aimed at balancing the distribution of classes in the training data. Resampling techniques are employed for this purpose and they are divided into oversampling [15], undersampling [16] and hybridsampling methods [17]. Generative Adversarial Networks [26] are machine learning 
models which are used to produce novel instances of samples from a targeted data distribution. A GAN variant CGAN [28] can be used to provide class labels to the GAN model to learn a distribution of a dataset conditioned on its class labels.

In this dissertation, we try to utilize CGAN as an efficient oversampling technique in the network intrusion detection system. A novel algorithm Adversarial Environment Generative Network (AEGAN), has been proposed in this study. It is a hybrid model that consists of AE-RL and CGAN. It is a combination of algorithm level approach (AE-RL) and data-level approach (CGAN). The combination of these two frameworks is able to provide an improved performance in the network intrusion detection system. Furthermore, a comparative study has been shown between CGAN and other oversampling techniques and a detailed analysis has been conducted over which oversampling techniques are more appropriate to combine with AE-RL. We conduct our experiments on the AWID dataset, comparing various oversampling techniques in their performance for classification over an imbalanced distribution of classes.

\subsection{AEGAN Details}

In general, AE-RL works well on imbalanced multi-class classification problems because of its ability to select samples in a balanced way. However, when there are very few samples in a minority class, even AE-RL suffers from a lack of variation in the data. We aim to solve this problem by creating relevant synthetic samples of the minority classes using CGAN. The CGAN model trains on the original dataset using the labels associated with each class as the conditional information. After this training, we select minority classes that are in need of oversampling and then produce new records by passing random noise to the CGAN combined with the class label to indicate what class to generate. CGAN is excellent at mimicking the original data 
distribution. Figure 5.1 illustrates the algorithm architecture of the proposed intru-

sion detection system. At first, various basic classifiers are trained on the original training dataset in order to gain general exploration knowledge. By observing each of the performance metric, we identify the minority classes that are most in need of augmentation. Then, we resample the chosen minority classes using CGAN. This phase is considered as the data-level portion of the solution to the class-imbalance problem. The oversampling process is used to raise the number of instances in the minority classes to levels comparable to the majority class. Both original and synthetic records are combined into a new training dataset. This modified dataset is then passed in as input for classifier training. In this dissertation, various classifiers are used for a comparative study. Apart from AE-RL, which is expected to tackle the class-imbalance problem on the algorithm-level, other classifiers are used to test the ability of CGAN to tackle the data imbalance problem. This also enables us to examine whether the algorithm-level approach has a countereffect on the data-level approach. From the probabilistic family, we have selected Naïve Bayes. Then, Random forest is used as an ensemble techniques. We have selected a Multi Layer Perceptron as an implementation of neural network. Finally, Logistic Regression from the linear model family is selected.

\subsection{Experimental Setup}

In this study, three oversampling techniques are studied: two traditional oversampling techniques SMOTE and ADASYN, as well as CGAN. The default parameters are used for both SMOTE and ADASYN. In CGAN there are two main modules: a generator and a discriminator, each of which is embodied by a neural network. The generator has four hidden layers; the number of output units in each layer are 32,64, 128 and 256, respectively. Similarly, the discriminator has four hidden layers with 256, 128, 


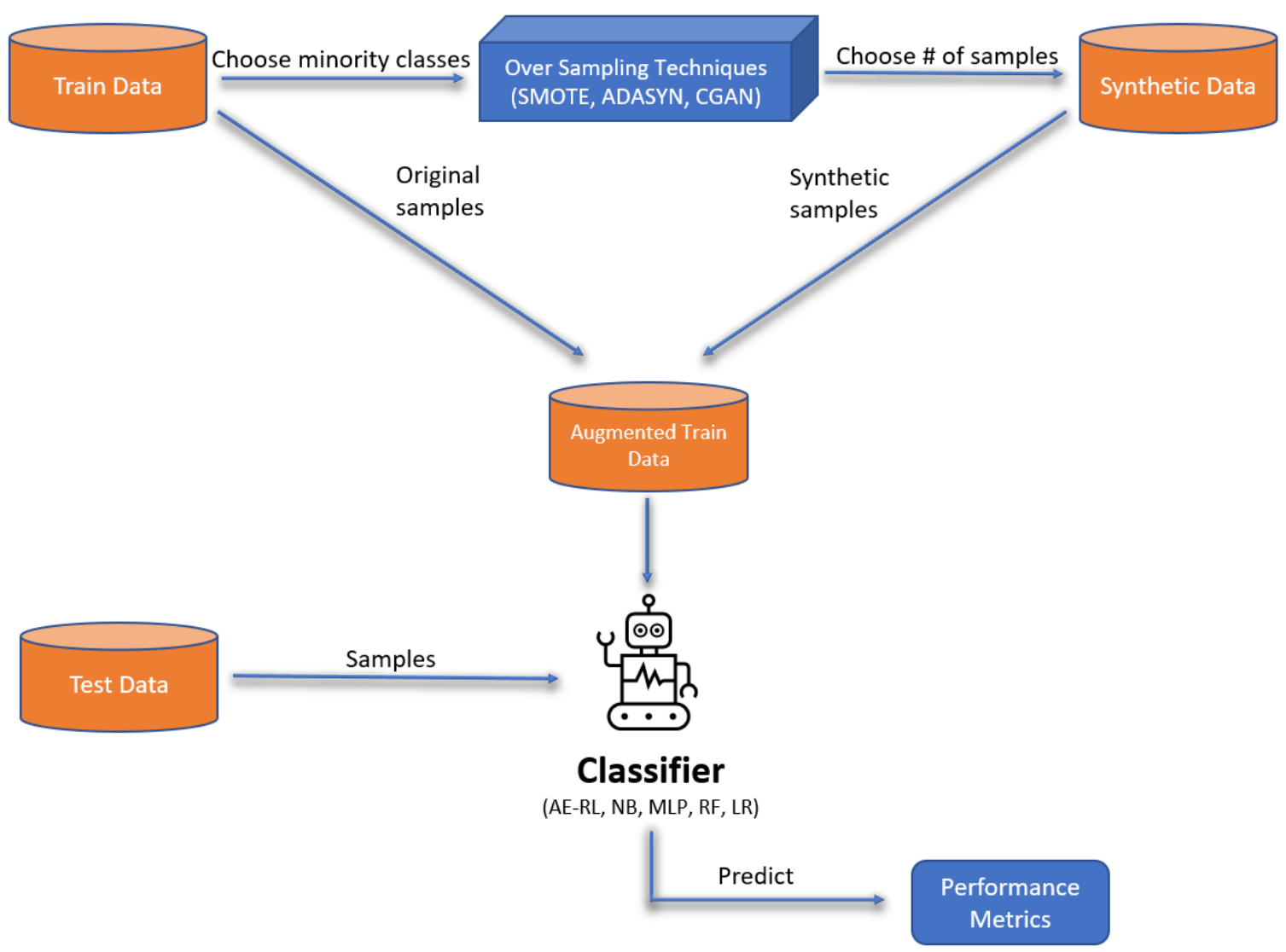

Figure 5.1: Algorithm architecture of the detection system

64 and 32 output units. The SGD optimizer is used along with 30000 steps. During the training process, the generator and discriminator take turns running training steps to update their parameters. In these initial experiments, we have configured each network to run a single training step on its turn. In our expanded experiments (Section 6.3), we investigate how the number of generator steps may be altered to achieve a more stable training process by better balancing the learning progress of the networks. A learning rate of 0.0001 is set to help CGAN learn smoothly. For the CGAN model, both the generator and discriminator accept class labels as conditional information along with their standard input features. After the training stage, the generator can be employed to create synthetic samples of a specific class. After initial experimentation, we have chosen to use a 100-dimensional vector of Gaussian noise 
as the input for the generator network.

There are four classes in AWID dataset: Normal, Injection, Flooding and Impersonation. We observe that the injection class has a unique distribution and results in $100 \%$ detection rate by all studied classifiers. Since injection class has nearly 100\% detection rate we have decided to create synthetic samples only for the flooding and impersonation classes. It is crucial to decide the number of synthetic samples to create. We have created 1 million synthetic samples for each of the flooding and impersonation classes as this not only balances the distribution, but also provides enough variation to these minority classes to assists classifiers like AE-RL to be most effective.

In this dissertation, the main machine learning classifier used is AE-RL. It is a complex classifier to manipulate. But it offers great potential for handling an imbalanced dataset. The parameters of AE-RL are set up as described in [25]. Other standard machine learning algorithms (NB, RF, MLP, LR) are also included in our experiments in order to compare their results with those of AE-RL. The results of these standard classifiers are recorded for usage both with and without oversampling techniques. A detailed comparison is shown in the Experimental Results \& Analysis section of this chapter. Except AE-RL, all other classifiers are implemented using the scikit learn. For these classifiers, default parameters are used, and all experiments are performed on virtual nodes with the same level of computational power. For each combination of a classifier and an oversampling technique, we perform the experiment 10 times and report the average value of our selected evaluation metrics as the result. The reason for adding average results is that machine learning classifiers provide a little bit of varied results on each run due to their internal mechanism. 


\subsection{Experiment Results \& Analysis}

In this section, we present the results of all classifiers when applied with different oversampling techniques. We report all four-evaluation metrics and provide analysis on these results.

Table 5.1: Results of AE-RL on AWID

\begin{tabular}{ccccc} 
Method & F1-score & Accuracy & Precision & Recall \\
\hline AE-RL & 0.9334 & 0.9289 & 0.9415 & 0.9289 \\
AE-RL + SMOTE & 0.9337 & 0.9388 & 0.9398 & 0.9388 \\
AE-RL + ADASYN & 0.9320 & 0.9402 & 0.9155 & 0.9578 \\
AE-RL + CGAN & $\mathbf{0 . 9 4 3 8}$ & $\mathbf{0 . 9 3 7 1}$ & $\mathbf{0 . 9 5 9 2}$ & $\mathbf{0 . 9 3 7 1}$ \\
\hline
\end{tabular}

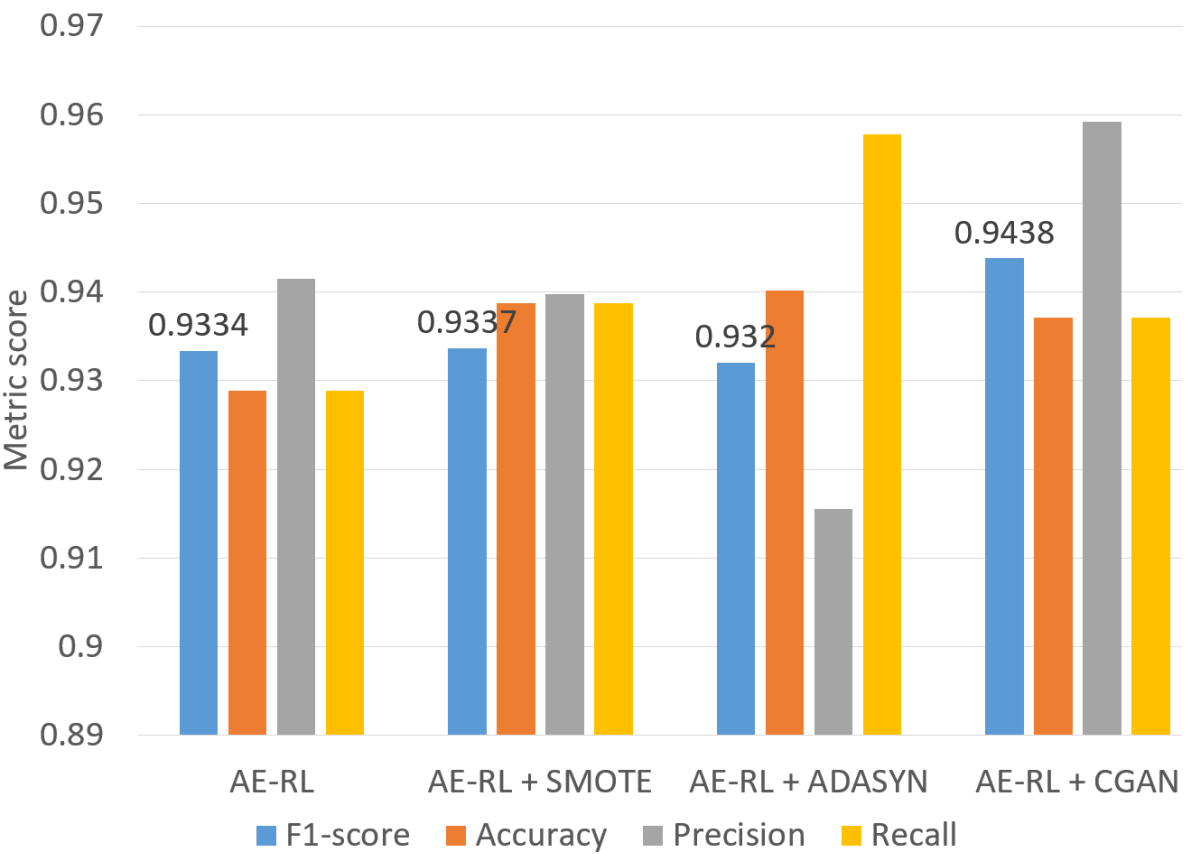

Figure 5.2: Various oversample techniques applied on AE-RL

Table 5.1 and Figure 5.2 illustrate the performance of AE-RL combined with various oversampling techniques. We observe that AE-RL + CGAN achieves the best F1-score of 0.9438, while AE-RL combined with SMOTE also produces results 
that outperform the original AE-RL. Similarly, AE-RL combined with ADASYN achieves a similar improved performance. Since AE-RL takes a unique algorithmlevel approach to resolve class-imbalance, we were expecting a countereffect of this approach on the overall performance. However, results show that despite the small amount of countereffect observed (as presented later in this subsection), it is beneficial to further add a data-level resampling phase into the AE-RL classifier.

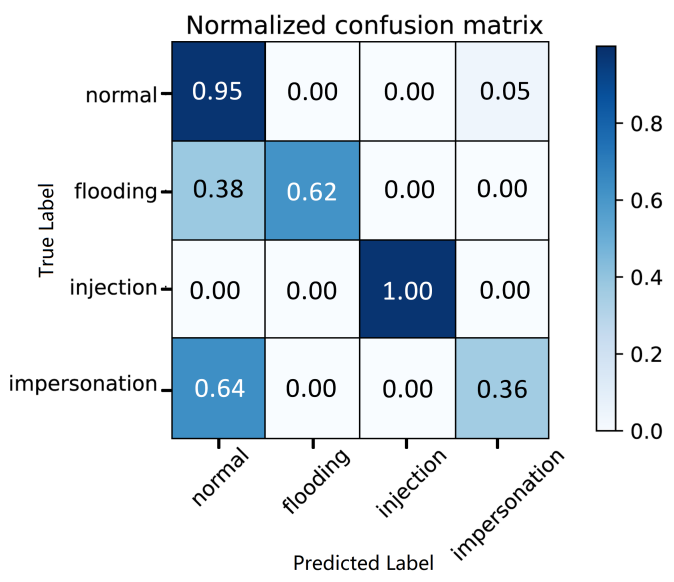

(a) AE-RL

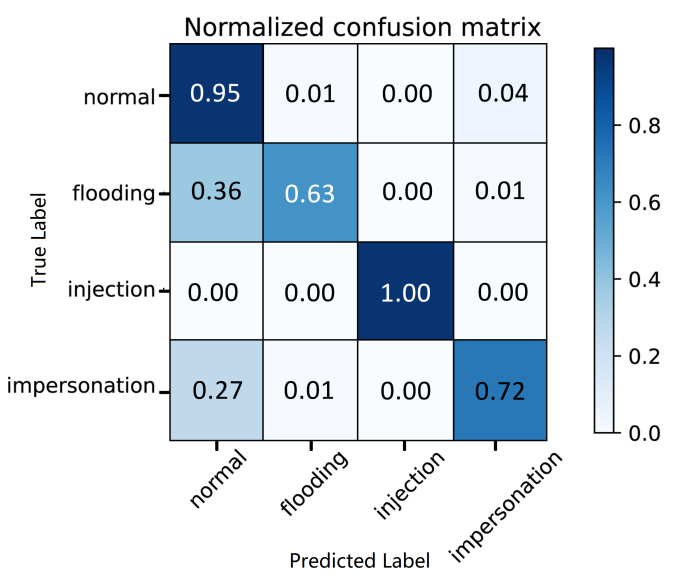

(b) AEGAN

Figure 5.3: Confusion Matrices of AE-RL \& AEGAN.

Figure 5.3 is used to show a comparison between the original AE-RL and the case when CGAN is combined with AE-RL. A Normalized Confusion Matrix (NCM) is used to illustrate the results. First of all, we observe an improvement on the prediction of the minority classes: the correct labelling of the Flooding attack class is increased from $62 \%$ to $63 \%$ and the correct labelling of the Impersonation class is increased from $36 \%$ to $72 \%$. Meanwhile, the false-positive rate on the Normal class labelled as the Impersonation class is drastically decreased from $64 \%$ to $27 \%$. As an effect of these shifted percentages, the AE-RL classifier gains an overall performance improvement from $93.34 \%$ to $94.38 \%$ in terms of the F1-score.

Table 5.2 and Figure 5.4 illustrate the performance of Naive Bayes with various 
Table 5.2: Results of Naive Bayes on AWID

\begin{tabular}{clclc} 
Method & F1-score & Accuracy & Precision & Recall \\
\hline NB & 0.8925 & 0.8734 & 0.9154 & 0.8726 \\
NB + SMOTE & 0.8912 & 0.8731 & 0.9125 & 0.8714 \\
NB + ADASYN & 0.8757 & 0.8235 & 0.9648 & 0.8232 \\
NB + CGAN & $\mathbf{0 . 9 4 2 8}$ & $\mathbf{0 . 9 5 0 4}$ & $\mathbf{0 . 9 3 5 4}$ & $\mathbf{0 . 9 5 0 4}$ \\
\hline
\end{tabular}

1

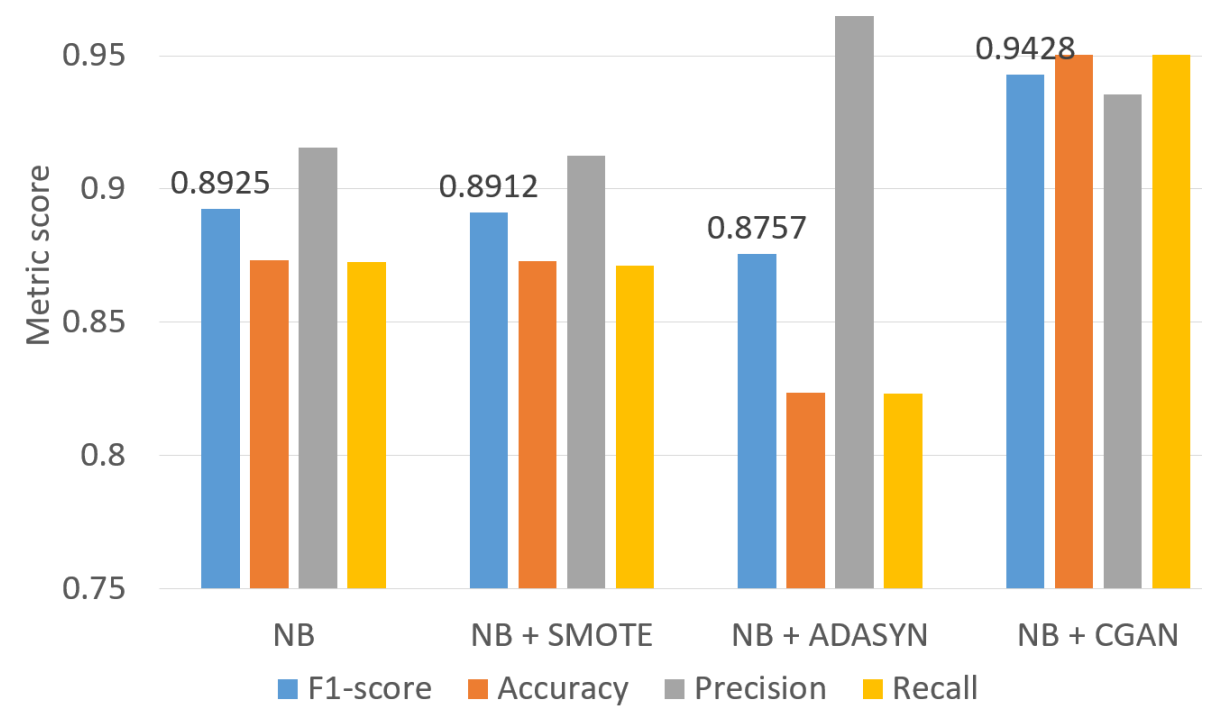

Figure 5.4: Various oversample techniques applied to Naive Bayes

oversampling techniques. NB combined CGAN achieves the best F1-score of 0.9428, while NB combined with SMOTE achieves similar performance to the original NB. Finally, NB combined with ADASYN receives the worst performance. Because NB is a probabilistic model, the distribution of the dataset has a great impact on the classification result. GAN is known for being great at mimicking the distribution of the original dataset whereas SMOTE and ADASYN generate synthetic samples based on distance measures. Therefore, CGAN+NB performs the best compared to applying SMOTE or ADASYN on NB. When the variable independence assumption is enforced, NB has an advantage of having a fast execution time by simply formulating 
predictions based on the conditional probability model. However, since there is no control put on the augmented portion of the training data, the newly generated samples might change the feature independence. Without this variable independence assumption, the overall performance is not guaranteed.

Table 5.3: Results of MLP on AWID

\begin{tabular}{clcll} 
Method & F1-score & Accuracy & Precision & Recall \\
\hline MLP & 0.9245 & 0.9324 & 0.9215 & 0.9367 \\
MLP + SMOTE & 0.9354 & 0.9314 & 0.9347 & 0.9385 \\
MLP + ADASYN & 0.9158 & 0.9226 & 0.9274 & 0.9287 \\
MLP + CGAN & $\mathbf{0 . 9 5 2 3}$ & $\mathbf{0 . 9 5 1 6}$ & $\mathbf{0 . 9 5 7 4}$ & $\mathbf{0 . 9 5 1 6}$ \\
\hline
\end{tabular}

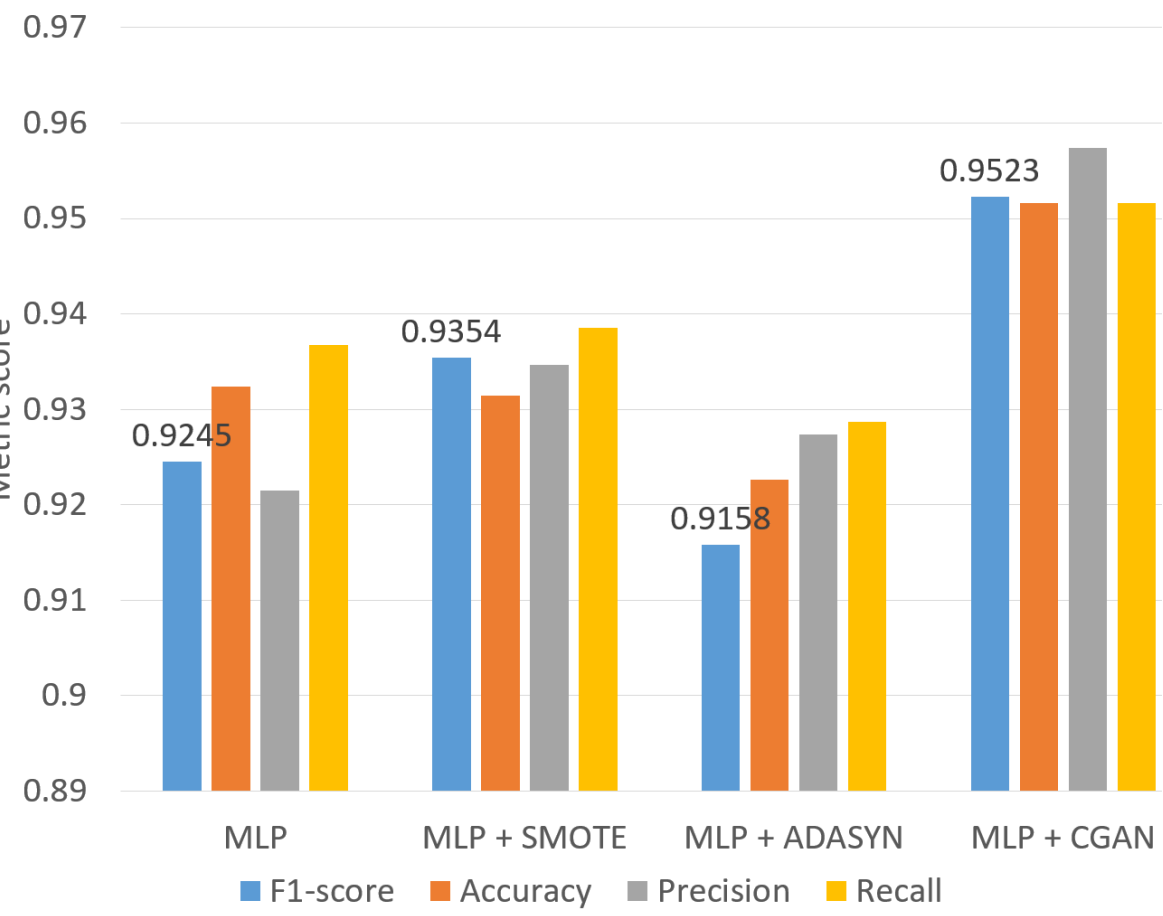

Figure 5.5: Various resample techniques applied to MLP

Table 5.3 and Figure 5.5 illustrate the performance of Multilayer Perceptron with various oversampling techniques. The best performance is shown when MLP is combined with CGAN. This combination achieves an F1-score of 0.9523. When MLP 
is combined with SMOTE, its F1-score ends up reaching 0.9354. When MLP and ADASYN are combined, a slightly worse performance is shown with an F1-score as 0.9158. Although the base AE-RL has a better F1-score than the base MLP, this reverses when applying oversampling, resulting in better performance with MLP combined with CGAN. This demonstrates that applying a data-level approach on a normal classifier can achieve a better result than applying it on an algorithm-level approach.

Table 5.4: Results of Random Forest on AWID

\begin{tabular}{ccclc} 
Method & F1-score & Accuracy & Precision & Recall \\
\hline RF & 0.9144 & 0.9368 & 0.9347 & 0.9359 \\
$\mathrm{RF}+\mathrm{SMOTE}$ & 0.8921 & 0.9238 & 0.9245 & 0.9234 \\
$\mathrm{RF}+$ ADASYN & 0.9242 & 0.9548 & 0.9551 & 0.9548 \\
$\mathbf{R F}+\mathbf{C G A N}$ & $\mathbf{0 . 9 2 8 2}$ & $\mathbf{0 . 9 4 7 3}$ & $\mathbf{0 . 9 5 7 3}$ & $\mathbf{0 . 9 4 8 7}$ \\
\hline
\end{tabular}

0.98

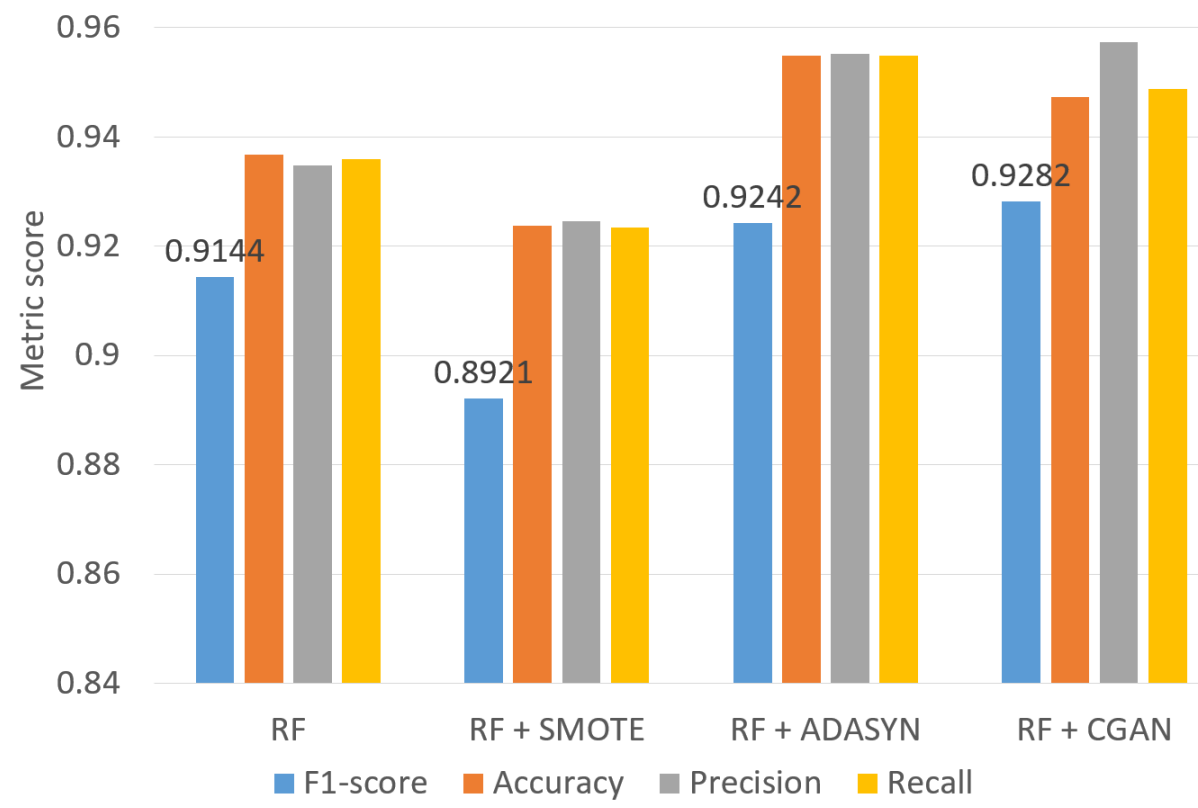

Figure 5.6: Various oversampling techniques applied to RF 
Table 5.4 and Figure 5.6 illustrate the performance of Random Forest when various oversampling techniques are applied. The highest F1-score is achieved by combining RF with CGAN. Its F1-score is 0.9282. When RF is combined with ADASYN, it achieves a better performance than the original RF with F1-score reaching 0.9242. Since RF is an ensemble of decision trees, it takes the majority decision from the subtrees. When combined with ADASYN, it focuses on the hard-to-learn minority samples, which slightly improves the result. We observe similar results when using the augmented dataset produced using CGAN because it captures the distribution of the minority classes well, which helps to improve the detection rate of the minority classes. On the other hand, the SMOTE-augmented dataset led to worse performance than that of the original dataset. Our intuition is that the augmented dataset creates a bias towards the minority classes that ends up misclassifying some normal class instances. However, further experiments are required to quantify this bias. We will address this in future work.

Table 5.5: Results of Logistic Regression on AWID

\begin{tabular}{clcll} 
Method & F1-score & Accuracy & Precision & Recall \\
\hline LR & 0.9569 & 0.9586 & 0.9663 & 0.9586 \\
LR + SMOTE & 0.9568 & 0.9503 & 0.9712 & 0.9503 \\
LR + ADASYN & 0.9521 & 0.9522 & 0.9557 & 0.9522 \\
LR + CGAN & $\mathbf{0 . 9 8 5 0}$ & $\mathbf{0 . 9 8 4 9}$ & $\mathbf{0 . 9 8 5 1}$ & $\mathbf{0 . 9 8 4 9}$ \\
\hline
\end{tabular}

Table 5.5 and Figure 5.7 illustrate the performance of Logistic Regression when various oversampling techniques are applied. An outstanding performance is achieved when LR is combined with CGAN, reaching an F1-score of 0.985, while LR combined with SMOTE achieves similar performance as the original LR with F1-scores of 0.9568 and 0.9569 , respectively. When LR is combined with ADASYN, its performance is comparatively poor reaching a F1-score of 0.9521 . We can observe that the overall 


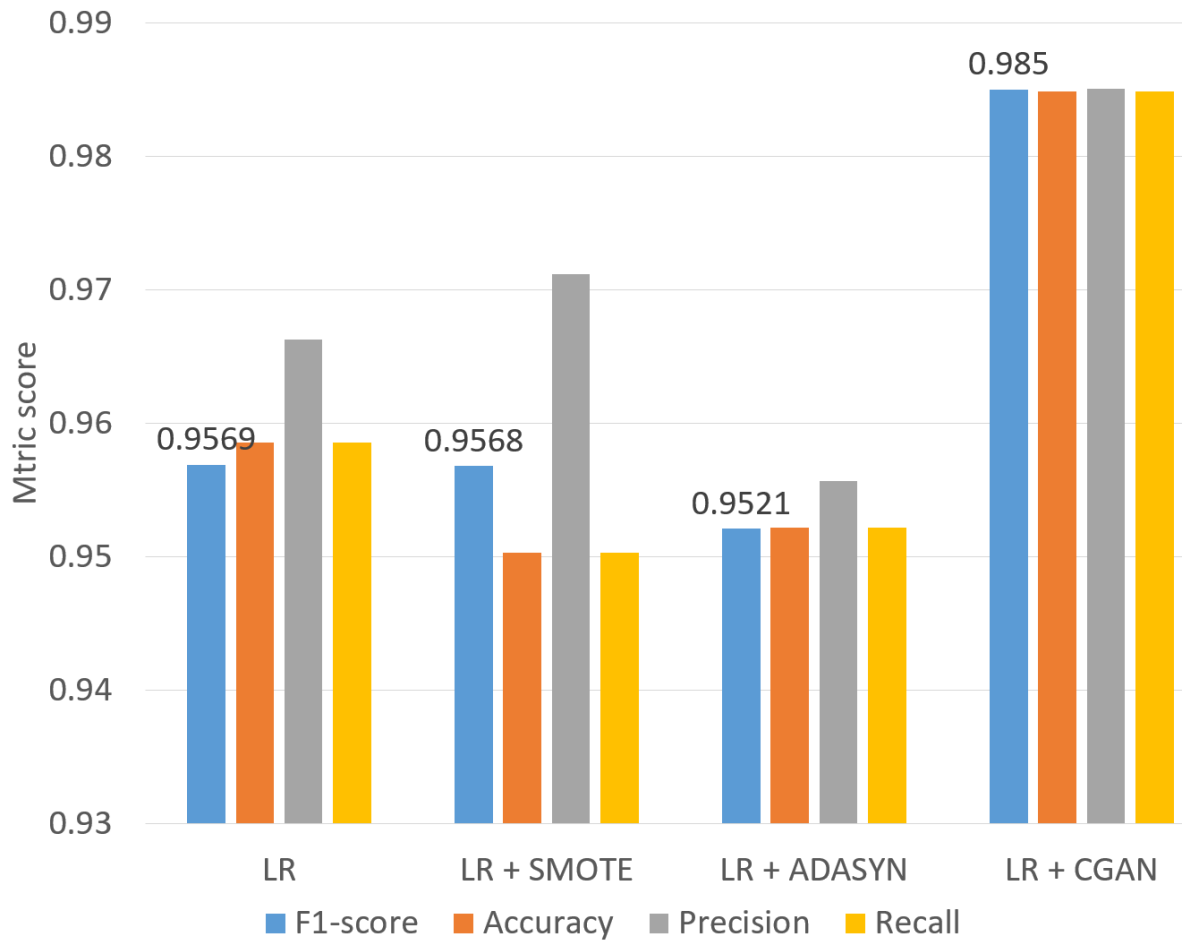

Figure 5.7: Various oversampling techniques applied to LR

performance of LR-based classifiers outperforms all other classifiers.

We illustrate a comparison between LR and LR + CGAN using NCM in Figure 5.8. We observe a significant improvement on one of the minority classes, Flooding: the rate of successful identification of this type of attack is increased from $11 \%$ to $62 \%$ while eliminating $51 \%$ of the false-positive rate on predicting as Normal. Meanwhile, the majority class Normal gains a further improvement in performance from $97 \%$ to $99 \%$ while eliminating $3 \%$ of false-positive rate on the Impersonation attack class.

In summary, all studied oversampling techniques, namely, SMOTE, ADASYN and CGAN have a positive impact in most of the cases over AE-RL, MLP, RF and LR. A crucial observation is that CGAN always performs better than other oversampling techniques. Furthermore, all classifiers combined with CGAN consistently outperform their original models. Among these results, LR demonstrates the most significant improvement when combined with CGAN. 


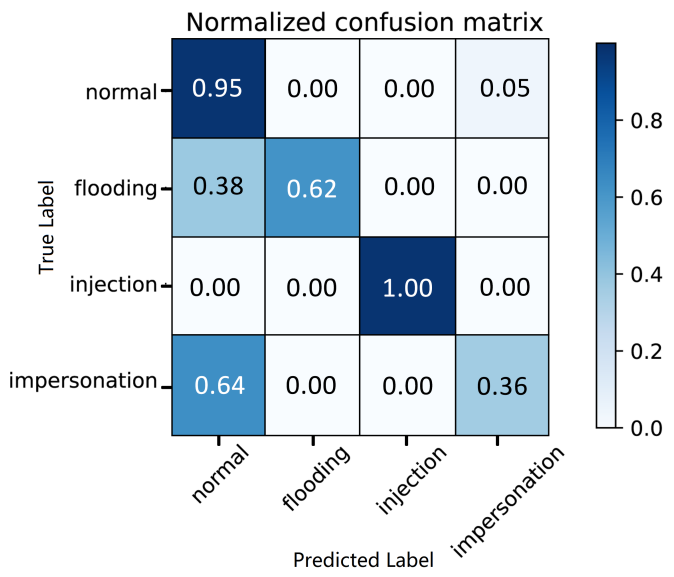

(a) Logistic Regression without oversampling Data

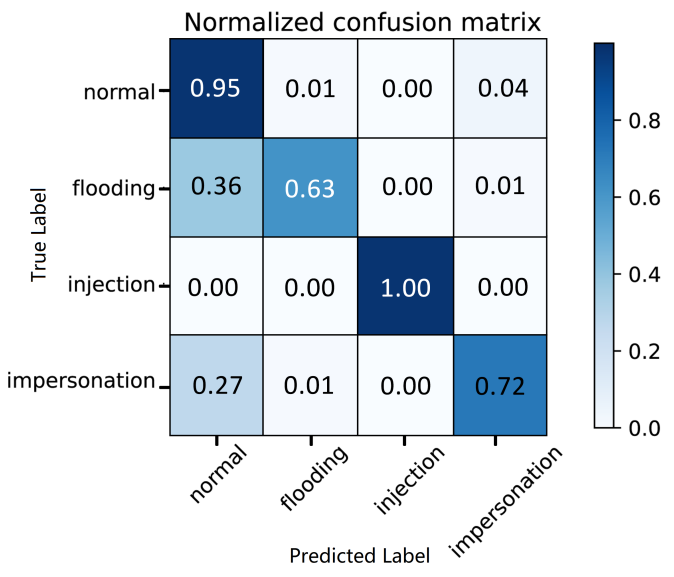

(b) Logistic Regression + CGAN Data

Figure 5.8: Confusion Matrices of Logistic Regression with and without CGAN.

Table 5.6: Population variance on the F1-scores for each classifier

\begin{tabular}{cl} 
Method & $\sigma^{2}$ \\
\hline AE-RL & 0.000022146 \\
NB & 0.000638703 \\
MLP & 0.000185585 \\
RF & 0.000195837 \\
LR & 0.000169525 \\
\hline
\end{tabular}

Lastly, we calculate the population variance for each classifier to inspect the variation in performance induced by oversampling. These measures are calculated using the F1-scores from the base classifiers and each of the their oversampling methods. The formula of population variance is as follows:

$$
\sigma^{2}=\frac{\sum_{i=1}^{n}\left(x_{i}-\mu\right)^{2}}{n}
$$

where $x_{i}$ is the sample from each classifier and $\mu$ is the mean of the corresponding population. From the results shown in Table 5.6, we observe that AE-RL has the lowest variance value of 0.000022146 when applying oversampling techniques. Lower 
variance values are indicative of less significant changes in the performance of a classifier from the use of oversampling. This demonstrates that data-level approaches have a less significant impact on algorithm level approaches compared to other classifiers. Since the algorithm level approach addresses the imbalance problem of the dataset, its effect is not as substantial as the effect of other classifiers when data level approaches are applied. On the other hand, NB has the highest variance value of 0.000638703 after applying oversampling techniques. This is due to NB's rigid assumption of independence, which cannot be avoided in the augmented portion of the training data. 


\section{Chapter 6}

\section{Further Improvement}

In this chapter, we extend the experimental methodology in order to define a more robust set of experiments. Additionally, we include NSL-KDD as a second dataset on which to validate oversampling techniques when dealing with very few minority samples. Though both AWID and NSL-KDD datasets are imbalanced datasets, the minority classes of the AWID dataset have a more moderate number of samples than the minority classes of the NSL-KDD dataset. We provide analysis and discussion on the impact of using oversampling techniques over these two datasets.

\subsection{Executing AE-RL on NSL-KDD}

AE-RL works differently than other selected classifiers. It learns based on the interaction with the environment. Furthermore, NSL-KDD has a different data distribution than the AWID dataset. In the NSL-KDD dataset, minority classes like R2L and U2R have extremely low samples. Due to this low number of samples, simply feeding the oversampled NSL-KDD dataset to AE-RL will cause AE-RL to select synthetic samples without using original samples in the training phase. In [37], the authors pro-

vide a strategy to insert new synthetic samples to AE-RL as soon as one sample of R2L or U2R is reused, in order to make sure AE-RL uses the original samples before 
synthetic samples in the training period. We propose a refined selection technique adopting this idea to propose a refined selection technique. In [37], the oversampling was done on the subclasses of 2 minority classes while training. It increased the training time of AE-RL. On the contrary, in our model, we oversample based on the parent classes. And we also perform oversampling before the training to achieve faster training time. We chose not to conduct oversampling at the level of subclasses on NSL-KDD to keep AE-RL setup as consistent as possible for both datasets. To facilate a better understanding of this selection process, we describe the details in algorithm 1 .

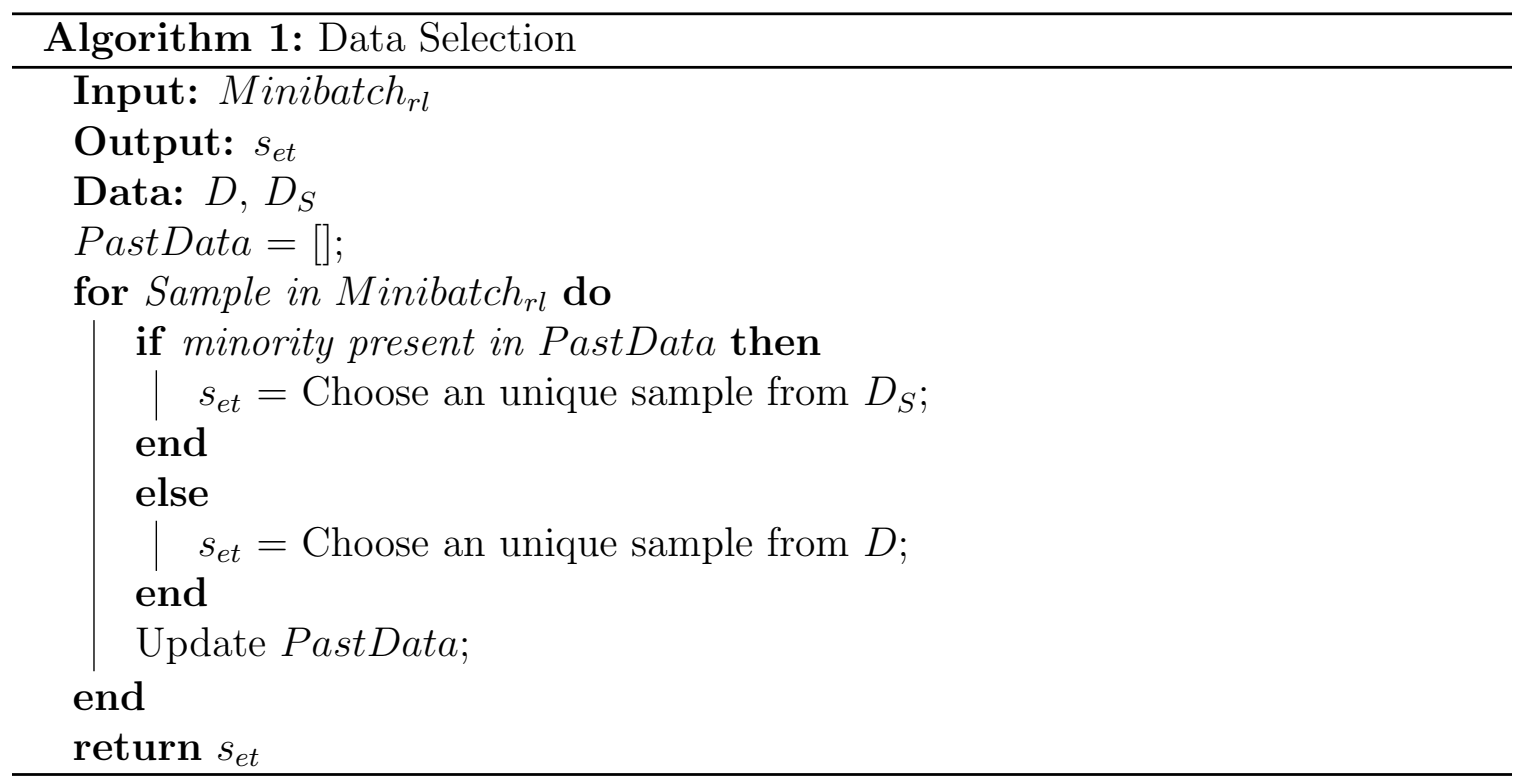

Here Minibatch $_{r l}$ is the batch provided by the environment agent. $D$ is the original dataset, and $D_{S}$ is the set of synthetic samples. PastData is the array to keep track of incoming samples. 


\subsection{CGAN vs. WCGAN}

Our previous research on oversampling used CGAN as an oversampling technique to solve the network intrusion detection system's imbalance problem. We also investigated another GAN variant, such as Wasserstein Conditional Generative Adversarial Network (WCGAN), as an oversampling technique. it is a GAN variant using an alternate loss function during the training process based on Earth Mover's distance to measure the distance between the distributions of the generated and real data. The advantage of this GAN is that it improves training stability and lead to better quality generators [83].

Table 6.1: Accuracy and F1-score of CGAN and WCGAN with different classifiers

\begin{tabular}{|c|c|c|c|c|}
\hline & \multicolumn{2}{|c|}{ CGAN } & \multicolumn{2}{c|}{ WCGAN } \\
\hline Method & Accuracy & F1-score & Accuracy & F1-score \\
\hline AE-RL & 0.9371 & 0.9438 & 0.9301 & 0.9285 \\
\hline NB & 0.9504 & 0.9428 & 0.9777 & 0.9726 \\
\hline MLP & 0.9516 & 0.9523 & 0.9321 & 0.9369 \\
\hline RF & 0.9473 & 0.9282 & 0.9507 & 0.9323 \\
\hline LR & 0.9849 & 0.9850 & 0.9223 & 0.8856 \\
\hline
\end{tabular}

In Table 6.1, the Accuracy and F1-score of CGAN and WCGAN have been shown across all the classifiers on AWID dataset. AE-RL, MLP, LR all attain better performance with CGAN compared to WCGAN in terms of Accuracy and F1-score. NB seems to perform very well in terms of Accuracy and F1-score with WCGAN, but we observe that in the case of NB with WCGAN, all the predictions of the Flooding class are shifted to normal class resulting in the poor performance of detecting minority classes. Since normal class is the majority class, we get excellent results in terms of Accuracy and F1-score. This is why we cannot consider NB's performance as a good result here. RF also performs better with WCGAN compared to CGAN. But 
the improvement is very low, and RF is also one of the lowest performing classifiers among all other classifiers. So based on these obtained results, CGAN is a better choice as an oversampling technique.

\subsection{Variance of The CGAN}

CGAN has a complicated architecture compared to other oversampling techniques. Due to this complicated architecture, it provides highly varied synthetic samples on every run. Since there is a variation in the created synthetic samples, it has been seen that it adds more additional variance in the results provided by Machine Learning classifiers compared to other oversampling techniques. In chapter 5 , a single set of synthetic samples were used per oversampling method for all runs over which classifier results were averaged. In order to achieve more robust results, we propose to use different synthetic samples created by oversampling techniques for each run. Since CGAN is more computationally expensive than other oversampling techniques, we trained 10 different instances of the CGAN model prior, each of which is used to create a set of synthetic samples for each run. Then, we report the average results of those runs. In this way, the reported results include variance that comes from machine learning classifiers and variance that arises from using CGAN. In previous experiments (section 5.4), we have used a single generator step during the CGAN training process. Here, we study the impact of changing this value on the performance of the CGAN. In Table 6.2, standard deviation of the F1-score has been shown for different generator steps. Here, LR has been chosen as a classifier because it is the best classifier from our previous study. With 1 generator step, the average F1-score is 0.9359 and $\sigma$ is 0.069150 . Since It has a low average score and high $\sigma$, the obtained result is not as good as expected. But with generator steps $=2$ Average F1-score is 0.9713 and $\sigma$ is 0.007476 . It clearly shows that with generator steps $=2$, it has a 
very high average with a very low $\sigma$ which is more robust than step 1 . We further experiment with step 3 , but it has been seen that the average result has decreased and $\sigma$ has increased, so we didn't further test for more step numbers. This step-changing has been applied to achieve a better balance of performance between two networks (generator \& discriminator) in the CGAN architecture. And this modification is done specifically for the AWID dataset.

Table 6.2: Standard Deviation of the F1-score of LR on different generator steps (AWID Dataset)

\begin{tabular}{|c|c|c|}
\hline & Average & $\sigma$ \\
\hline Generator Steps $=1$ & 0.9359 & 0.069150 \\
\hline Generator Steps $=2$ & 0.9713 & 0.007476 \\
\hline Generator Steps $=3$ & 0.9512 & 0.021386 \\
\hline
\end{tabular}

We have additionally tested the impact of generator step selection for the NSLKDD dataset with LR. In Table 6.3, the average and $\sigma$ of the F1-score is shown for the NSL-KDD dataset. With the NSL-KDD dataset, the generator is already performing well when using a single step of training per generator step. After changing the step from 1 to 2, the average result remains almost the same, and $\sigma$ increases slightly. Given this, we have chosen to keep the setting at a single step for the NSL-KDD dataset.

Table 6.3: Standard Deviation of the F1-score of LR on different generator steps (NSL-KDD Dataset)

\begin{tabular}{|c|c|c|}
\hline & Average & $\sigma$ \\
\hline Generator Steps $=1$ & 0.706731 & 0.001072 \\
\hline Generator Steps $=2$ & 0.706746 & 0.001103 \\
\hline
\end{tabular}




\subsection{Experiments with The Number of Synthetic Samples}

With any oversampling technique, it is crucial to select an appropriate number of synthetic samples to create. Generating too few synthetic samples may fail to address imbalance problem of the dataset. Yet, creating too many synthetic samples may overfit classifiers. In chapter 5, 1 million synthetic samples were generated for the class Impersonation and Flooding classes in the AWID dataset. To study the impact of the number of synthetic samples on classifier performance, we incorporate a range of sample sizes into our experiments. More specifically, we select sample sizes as fixed ratios of the number of samples in the majority class. The formula of ratio is given in $(6.1)$.

$$
\text { Ratio }=\frac{\text { number of oversampled minority class }}{\text { number of majority class }}
$$

For example, a ratio of $50 \%$ indicates that synthetic samples will be added to a minority class such that its total number of samples (original plus synthetic) is equal to $50 \%$ of the samples in the majority class. For the AWID dataset, we have selected ratios of $5 \%, 10 \%, 20 \%, 50 \%, 100 \%$. Here, oversampling is only done on Impersonation and Flooding (as in the previous experiments).

In the NSL-KDD dataset, there are 4 different types of cyber-attacks. All of them have fewer samples than the normal class. Among these 4 classes, DoS and Probe have more samples than R2L and U2R. The classes that are oversampled at each ratio are shown in Table 6.4. For ratios of $5 \%$ and $10 \%$, only R2L and U2R are oversampled because DoS and Probe already have more samples than required to reach these ratios. After $50 \%$, all of the 4 classes are oversampled. We have introduced an additional ratio of $75 \%$ to analyze the effects of the oversampling techniques on specific classes (discussed later). 
Table 6.4: Oversampled Minority Classes for a Specific Ratio

\begin{tabular}{cl} 
Ratios & Minority Classes \\
\hline $5 \%$ & R2L, U2R \\
$10 \%$ & R2L, U2R \\
$20 \%$ & Probe, R2L, U2R \\
$50 \%$ & Probe, R2L, U2R \\
$75 \%$ & DoS, Probe, R2L, U2R \\
$100 \%$ & DoS, Probe, R2L, U2R \\
\hline
\end{tabular}

\subsection{Results \& Discussion}

In this section, we are going to present all experimental results for two different datasets. All of the ratios are being tested with each combination of classifier and oversampling technique. As explained in section 6.3, All the results are an average over 10 runs of machine learning algorithms on 10 different sets of synthetic samples. We mainly focus on the F1-score for analyzing the results.

\subsubsection{Analysis on the results of AWID Dataset}

In Table 6.5, the performance of AE-RL is given on different ratios. SMOTE with a ratio of $10 \%$ has achieved the highest F1-score for this classifier. It is interesting to note that for all oversampling techniques applied to AE-RL, we always obtain the best $\mathrm{F} 1$-score on a $10 \%$ ratio. We believe that the reason for this same peak for all of the oversampling techniques is that if we create excessive synthetic samples, AE-RL starts to use all the synthetic samples instead of using the original sample. It degrades the overall performance of the AE-RL instead of improving it. It can be stated that the ideal ratio for AE-RL classifier is $10 \%$ for all the oversampling techniques.

Performance of NB is shown in Table 6.6. In NB, the ratio has a minimal impact. For almost all ratios, the results are quite similar, especially for SMOTE. We note 
Table 6.5: Performance of AE-RL on Different Ratios (AWID)

\begin{tabular}{|c|c|l|l|l|l|}
\hline \multicolumn{2}{|c|}{} & Accuracy & F1-score & Precision & Recall \\
\hline \multicolumn{1}{|c|}{ Original } & 0.9289 & 0.9334 & 0.9415 & 0.9289 \\
\hline \multirow{5}{*}{ CGAN } & $5 \%$ & 0.9291 & 0.9376 & 0.9537 & 0.9291 \\
\cline { 2 - 6 } & $10 \%$ & 0.9229 & $\mathbf{0 . 9 5 7 6}$ & 0.9717 & 0.9445 \\
\cline { 2 - 6 } & $20 \%$ & 0.9388 & 0.9466 & 0.9628 & 0.9388 \\
\cline { 2 - 6 } & $50 \%$ & 0.9256 & 0.9320 & 0.9497 & 0.9256 \\
\cline { 2 - 6 } & $100 \%$ & 0.9332 & 0.9272 & 0.9350 & 0.9332 \\
\hline \multirow{5}{*}{ SMOTE } & $5 \%$ & 0.9425 & 0.9496 & 0.9665 & 0.9425 \\
\cline { 2 - 6 } & $10 \%$ & 0.9487 & $\mathbf{0 . 9 6 7 6}$ & 0.9487 & 0.9538 \\
\cline { 2 - 6 } & $20 \%$ & 0.9208 & 0.9292 & 0.9446 & 0.9208 \\
\cline { 2 - 6 } & $50 \%$ & 0.9418 & 0.9010 & 0.9418 & 0.9140 \\
\cline { 2 - 6 } & $100 \%$ & 0.9260 & 0.9187 & 0.9338 & 0.9260 \\
\hline \multirow{5}{*}{ ADASYN } & $5 \%$ & 0.9208 & 0.9318 & 0.9506 & 0.9208 \\
\cline { 2 - 6 } & $10 \%$ & 0.9356 & $\mathbf{0 . 9 4 5 1}$ & 0.9633 & 0.9356 \\
\cline { 2 - 6 } & $20 \%$ & 0.9381 & 0.9413 & 0.9527 & 0.9381 \\
\cline { 2 - 6 } & $50 \%$ & 0.9359 & 0.9348 & 0.9421 & 0.9359 \\
\cline { 2 - 6 } & $100 \%$ & 0.9412 & 0.9331 & 0.9448 & 0.9412 \\
\hline
\end{tabular}

that as more synthetic samples are added using CGAN, the F1-score starts to increase gradually. Since the improvement is very low, we investigated the confusion matrix to see the reason for the change. As more synthetic samples are added, most of the Flooding class is predicted as a normal class. This is the reason for such a high F1-score of NB with CGAN. But NB does perform well-detecting Impersonation (another oversampled class) in general, and for the 100\% ratio the detection rate of the Impersonation is $93 \%$.

In Table 6.7, the performance of RF is presented on different ratios. In all cases, oversampling techniques improve the performance of the classifiers. Only for $10 \%$ with CGAN is there a difference noted between ratios. Other than that, for other 
Table 6.6: Performance of NB on Different Ratios (AWID)

\begin{tabular}{|c|c|r|r|r|r|}
\hline \multicolumn{2}{|c|}{} & Accuracy & F1-score & Precision & Recall \\
\hline \multirow{5}{*}{ Original } & 0.8734 & 0.8925 & 0.9154 & 0.8726 \\
\hline \multirow{5}{*}{ CGAN } & $5 \%$ & 0.9717 & 0.9692 & 0.9678 & 0.9717 \\
\cline { 2 - 6 } & $10 \%$ & 0.9776 & 0.9725 & 0.9748 & 0.9776 \\
\cline { 2 - 6 } & $20 \%$ & 0.9794 & 0.9734 & 0.9785 & 0.9794 \\
\cline { 2 - 6 } & $50 \%$ & 0.9798 & 0.9735 & 0.9802 & 0.9798 \\
\hline \multirow{5}{*}{ SMOTE } & $100 \%$ & 0.9831 & $\mathbf{0 . 9 7 6 9}$ & 0.9811 & 0.9831 \\
\cline { 2 - 6 } & $5 \%$ & 0.8695 & 0.8874 & 0.9133 & 0.8695 \\
\cline { 2 - 6 } & $10 \%$ & 0.8698 & $\mathbf{0 . 8 8 7 6}$ & 0.9133 & 0.8698 \\
\cline { 2 - 6 } & $20 \%$ & 0.8699 & $\mathbf{0 . 8 8 7 6}$ & 0.9133 & 0.8699 \\
\cline { 2 - 6 } & $50 \%$ & 0.8699 & $\mathbf{0 . 8 8 7 6}$ & 0.9133 & 0.8699 \\
\hline & $100 \%$ & 0.8699 & $\mathbf{0 . 8 8 7 6}$ & 0.9133 & 0.8699 \\
\hline & $5 \%$ & 0.8307 & $\mathbf{0 . 8 7 8 8}$ & 0.9583 & 0.8307 \\
\cline { 2 - 6 } & $10 \%$ & 0.8279 & 0.8768 & 0.9580 & 0.8279 \\
\cline { 2 - 6 } & $20 \%$ & 0.8207 & 0.8716 & 0.9572 & 0.8207 \\
\hline & $50 \%$ & 0.8169 & 0.8686 & 0.9566 & 0.8169 \\
\cline { 2 - 6 } & $100 \%$ & 0.8174 & 0.8671 & 0.9551 & 0.8174 \\
\hline
\end{tabular}

ratios, results are quite similar to each other. RF with the other two standard oversampling techniques, SMOTE and ADASYN, shows similar types of results for each ratio. It can say that for an oversampling techniques like SMOTE and ADASYN number of synthetic samples does not have any impactful contribution to improve the performance that can be mentioned. It pertains only to the application of these techniques to RF with the AWID dataset.

Results for the experiments using the MLP are shown in Table 6.8. MLP with CGAN using a ratio of $50 \%$ has the highest achieved F1-score compared to all other presented results in this table. The F1-scores for CGAN oversampling increase with the number of synthetic samples, up to a peak at $50 \%$ ratio, after which it doesn't 
Table 6.7: Performance of RF on Different Ratios (AWID)

\begin{tabular}{|c|c|l|l|l|l|}
\hline \multicolumn{2}{|c|}{} & Accuracy & F1-score & Precision & Recall \\
\hline \multirow{5}{*}{ Original } & 0.9368 & 0.9144 & 0.9347 & 0.9359 \\
\hline \multirow{5}{*}{ CGAN } & $5 \%$ & 0.9454 & 0.9257 & 0.9470 & 0.9454 \\
\cline { 2 - 6 } & $10 \%$ & 0.9507 & $\mathbf{0 . 9 3 3 0}$ & 0.9522 & 0.9507 \\
\cline { 2 - 6 } & $20 \%$ & 0.9451 & 0.9252 & 0.9472 & 0.9451 \\
\cline { 2 - 6 } & $50 \%$ & 0.9426 & 0.9209 & 0.9448 & 0.9426 \\
\hline \multirow{5}{*}{ SMOTE } & $100 \%$ & 0.9489 & 0.9299 & 0.9505 & 0.9489 \\
\cline { 2 - 6 } & $5 \%$ & 0.9467 & $\mathbf{0 . 9 2 6 2}$ & 0.9489 & 0.9467 \\
\cline { 2 - 6 } & $10 \%$ & 0.9459 & 0.9249 & 0.9479 & 0.9459 \\
\cline { 2 - 6 } & $20 \%$ & 0.9449 & 0.9240 & 0.9471 & 0.9449 \\
\cline { 2 - 6 } & $50 \%$ & 0.9401 & 0.9167 & 0.9420 & 0.9401 \\
\hline & $100 \%$ & 0.9463 & 0.9249 & 0.9453 & 0.9463 \\
\hline & $5 \%$ & 0.9478 & $\mathbf{0 . 9 2 8 1}$ & 0.9504 & 0.9478 \\
\cline { 2 - 6 } & $10 \%$ & 0.9473 & 0.9273 & 0.9497 & 0.9473 \\
\cline { 2 - 6 } & $20 \%$ & 0.9452 & 0.9242 & 0.9482 & 0.9452 \\
\hline & $50 \%$ & 0.9457 & 0.9244 & 0.9486 & 0.9457 \\
\cline { 2 - 6 } & $100 \%$ & 0.9480 & $\mathbf{0 . 9 2 8 1}$ & 0.9506 & 0.9480 \\
\hline
\end{tabular}

improve anymore. MLP with ADASYN shows a decreasing trend after reaching its peak at $10 \%$. For the $100 \%$ ratio, MLP with ADASYN performs worse than the base MLP classifier. In the $100 \%$ ratio, both SMOTE and ADASYN perform worse than other ratios in their own group. It can be inferred that generating too many synthetic samples might work as a countereffect for a classifier like MLP.

Finally, we test the impact of different oversampling ratios with LR. Results are shown in Table 6.9. Previously we attained the best result using LR. Here also LR provides us excellent results when used with CGAN. LR performs the best with CGAN at a ratio of $20 \%, 50 \%$ and $100 \%$. Though LR seems relatively insensitive to the number of synthetic samples, it is still very important where state of the art 
Table 6.8: Performance of MLP on Different Ratios (AWID)

\begin{tabular}{|c|c|c|c|c|c|}
\hline & & Accuracy & F1-score & Precision & Recall \\
\hline Origin & & 0.9324 & 0.9245 & 0.9215 & 0.9367 \\
\hline & $5 \%$ & 0.9320 & 0.9286 & 0.9275 & 0.9320 \\
\hline & $10 \%$ & 0.9374 & 0.9339 & 0.9335 & 0.9374 \\
\hline CGAN & $20 \%$ & 0.9394 & 0.9384 & 0.9403 & 0.9394 \\
\hline & $50 \%$ & 0.9453 & 0.9406 & 0.9433 & 0.9453 \\
\hline & $100 \%$ & 0.9401 & 0.9391 & 0.9407 & 0.9401 \\
\hline & $5 \%$ & 0.9362 & 0.9335 & 0.9328 & 0.9362 \\
\hline & $10 \%$ & 0.9391 & 0.9367 & 0.9375 & 0.9391 \\
\hline SMOTE & $20 \%$ & 0.9352 & 0.9349 & 0.9373 & 0.9352 \\
\hline & $50 \%$ & 0.9275 & 0.9283 & 0.9341 & 0.9275 \\
\hline & $100 \%$ & 0.9240 & 0.9263 & 0.9378 & 0.9240 \\
\hline & $5 \%$ & 0.9215 & 0.9197 & 0.9270 & 0.9215 \\
\hline & $10 \%$ & 0.9262 & 0.9238 & 0.9333 & 0.9262 \\
\hline ADASYN & $20 \%$ & 0.9168 & 0.9110 & 0.9213 & 0.9168 \\
\hline & $50 \%$ & 0.9071 & 0.9053 & 0.9186 & 0.9071 \\
\hline & $100 \%$ & 0.9035 & 0.9031 & 0.9164 & 0.9035 \\
\hline
\end{tabular}

result is so high. After the $10 \%$ ratio, there is a minor improvement that helps the other three remaining ratios achieve one of the best results among all other results. As previously explained in Chapter 5, LR doesn't work well with ADASYN. The observed effect of the ratio supports that claim by showing a clear decreasing trend after adding additional synthetic samples using ADASYN. A small amount of synthetic samples using SMOTE helps LR improve its performance from the baseline, but further addition of synthetic samples is not beneficial for LR. 
Table 6.9: Performance of LR on Different Ratios (AWID)

\begin{tabular}{|c|c|l|l|l|l|}
\hline \multicolumn{2}{|c|}{} & Accuracy & F1-score & Precision & Recall \\
\hline \multirow{7}{*}{ Original } & 0.9586 & 0.9569 & 0.9663 & 0.9586 \\
\cline { 2 - 6 } CGAN & $5 \%$ & 0.9668 & 0.9677 & 0.9754 & 0.9668 \\
\cline { 2 - 6 } & $20 \%$ & 0.9669 & 0.9681 & 0.9760 & 0.9669 \\
\cline { 2 - 6 } & $50 \%$ & 0.9688 & 0.9703 & 0.9785 & 0.9688 \\
\cline { 2 - 6 } & $100 \%$ & 0.9689 & 0.9705 & 0.9788 & 0.9689 \\
\hline \multirow{5}{*}{ SMOTE } & $5 \%$ & 0.9666 & $\mathbf{0 . 9 6 9 3}$ & 0.9769 & 0.9666 \\
\cline { 2 - 6 } & $10 \%$ & 0.9643 & 0.9676 & 0.9770 & 0.9643 \\
\cline { 2 - 6 } & $20 \%$ & 0.9599 & 0.9639 & 0.9736 & 0.9599 \\
\cline { 2 - 6 } & $50 \%$ & 0.9532 & 0.9589 & 0.9718 & 0.9532 \\
\cline { 2 - 6 } & $100 \%$ & 0.9504 & 0.9570 & 0.9717 & 0.9504 \\
\hline & $5 \%$ & 0.9537 & $\mathbf{0 . 9 3 8 9}$ & 0.9269 & 0.9537 \\
\cline { 2 - 6 } & $10 \%$ & 0.9516 & 0.9382 & 0.9270 & 0.9516 \\
\cline { 2 - 6 } & $20 \%$ & 0.9474 & 0.9362 & 0.9266 & 0.9474 \\
\hline \multirow{5}{*}{ ADASYN } & $50 \%$ & 0.9463 & 0.9357 & 0.9266 & 0.9463 \\
\cline { 2 - 6 } & $100 \%$ & 0.9456 & 0.9355 & 0.9269 & 0.9456 \\
\hline
\end{tabular}




\subsubsection{Analysis on the Results of NSL-KDD Dataset}

In Chapter 4, we have presented results from preliminary tests on the NSL-KDD dataset using different resampling techniques. Here, we include the NSL-KDD dataset again to test our proposed AEGAN. As with the AWID dataset, a complete comparative analysis has been conducted with different classifiers and oversampling techniques on the NSL-KDD dataset. All the reported results are the average of 10 runs, and variances that add to the results because of classifiers and oversampling techniques are being considered here. Due to the more robust experimental setup employed in the present chapter, there are some differences between these results and those of Chapter 4.

There are five classes in the NSL-KDD dataset: Normal, DoS, Probe, R2L, and U2R. They have $67342,45928,11656,995,52$ samples in the training dataset, respectively. Then in the testing dataset, they have 9711, 7456, 2421, 2754, 200 samples. In the training data distribution, Normal stands out as the majority class, which creates an imbalance. It is crucial to determine which classes to oversample. Although it is common to oversample all minority classes, we have decided to oversample R2L and U2R only. An intuition about oversampling only two minority classes is that the other two minority classes have already enough samples for classification purposes. It may not be adequate to solve the imbalance problem of the dataset. We will provide an experimental analysis later for choosing R2L and U2R only.

The results in Table 6.10 show a large variation across the ratios for AE-RL with CGAN. CGAN achieves its highest score with a 0.7902 F1-score at a $75 \%$ ratio. SMOTE and ADASYN also achieve the highest score close to CGAN but at ratios of $50 \%$ and $100 \%$, respectively. Although CGAN results have a large variation for different ratios, SMOTE and ADASYN show very stable results. AE-RL with SMOTE has a very good precision score; it is always more than $80 \%$. 
Table 6.10: Performance of AE-RL on Different Ratios (NSL-KDD)

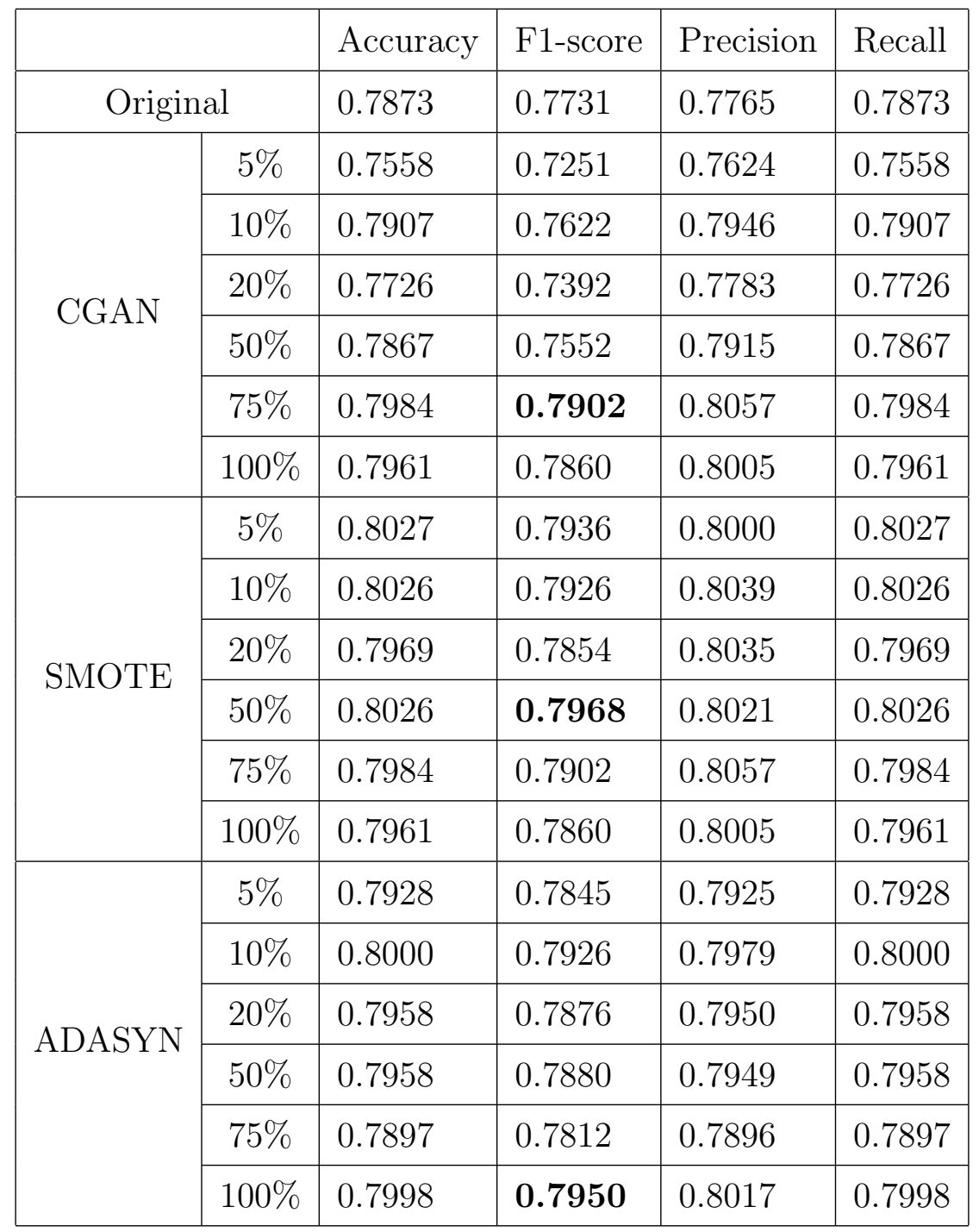

In Table 6.11, results are given for the performance of NB across the oversampling ratios on the NSL-KDD dataset. CGAN has a detrimental impact on NB's performance. The use of synthetic samples generated by CGAN has led to a large decrease in the f1-scores compared to the performance without oversampling. We believe that the reason for poor performance is the poor quality of synthetic samples which decreases the detection rate of minority classes. We believe the reason for the poor quality of synthetic samples is extremely low samples in two minority classes. 
The decreasing trend in performance as the oversampling ratio is increased provides evidence to this end. Contrarily, with SMOTE there is an increasing trend in performance as the ratios increase. SMOTE with a 100\% ratio achieves the highest F1-score 0.6360. NB with ADASYN is also shown an increasing trend and thus achieves the best F1-score with a ratio of $100 \%$. Despite this, the results still do not exceed those of the base result, showing that there is little impact from the oversampling technique.

Table 6.11: Performance of NB on Different ratios (NSL-KDD)

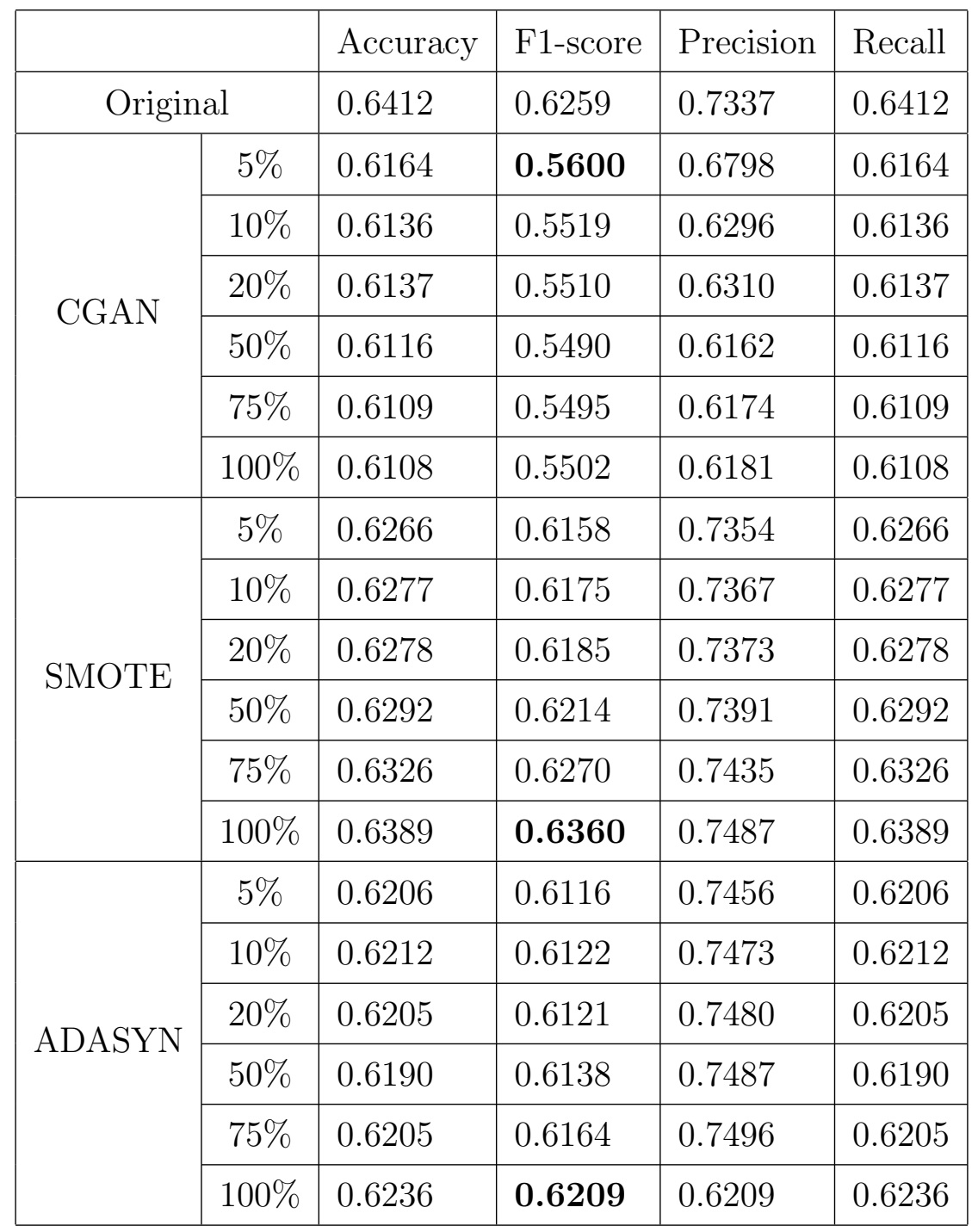

Results from the use of RF on different ratios for the NSL-KDD dataset are 
shown in Table 6.12. Here CGAN shows a similar result compared to the original result, and it beats the performance of base RF slightly with 50\% ratio. SMOTE and ADASYN have similar results. Both of them are able to increase the performance of $\mathrm{RF}$. Though improvement is minimal, it is still better to improve the existing classifier with additional synthetic data. SMOTE and ADASYN achieve their highest F1-score for $50 \%$ and $10 \%$, respectively.

Table 6.12: Performance of RF on Different ratios (NSL-KDD)

\begin{tabular}{|c|c|l|l|l|l|}
\hline \multicolumn{2}{|c|}{} & Accuracy & F1-score & Precision & Recall \\
\hline \multicolumn{1}{|c|}{ Original } & 0.7568 & 0.7171 & 0.8149 & 0.7568 \\
\hline \multirow{5}{*}{ CGAN } & $5 \%$ & 0.7561 & 0.7157 & 0.8145 & 0.7561 \\
\cline { 2 - 6 } & $10 \%$ & 0.7574 & 0.7180 & 0.8151 & 0.7574 \\
\cline { 2 - 6 } & $20 \%$ & 0.7548 & 0.7136 & 0.8128 & 0.7548 \\
\cline { 2 - 6 } & $50 \%$ & 0.7580 & $\mathbf{0 . 7 1 8 5}$ & 0.8156 & 0.7580 \\
\cline { 2 - 6 } & $75 \%$ & 0.7528 & 0.7125 & 0.8134 & 0.7528 \\
\cline { 2 - 6 } & $100 \%$ & 0.7536 & 0.7145 & 0.8132 & 0.7536 \\
\hline \multirow{5}{*}{ SMOTE } & $5 \%$ & 0.7531 & 0.7166 & 0.8156 & 0.7531 \\
\cline { 2 - 6 } & $10 \%$ & 0.7558 & 0.7214 & 0.8167 & 0.7558 \\
\cline { 2 - 6 } & $20 \%$ & 0.7574 & 0.7225 & 0.8169 & 0.7574 \\
\cline { 2 - 6 } & $50 \%$ & 0.7568 & $\mathbf{0 . 7 2 2 9}$ & 0.8146 & 0.7568 \\
\cline { 2 - 6 } & $75 \%$ & 0.7559 & 0.7222 & 0.8143 & 0.7559 \\
\cline { 2 - 6 } & $100 \%$ & 0.7564 & 0.7228 & 0.8149 & 0.7564 \\
\hline \multirow{5}{*}{ ADASYN } & $5 \%$ & 0.7559 & 0.7230 & 0.8159 & 0.7559 \\
\cline { 2 - 6 } & $10 \%$ & 0.7601 & $\mathbf{0 . 7 2 9 4}$ & 0.8183 & 0.7601 \\
\cline { 2 - 6 } & $20 \%$ & 0.7589 & 0.7287 & 0.8170 & 0.7589 \\
\cline { 2 - 6 } & $50 \%$ & 0.7581 & 0.7269 & 0.8170 & 0.7581 \\
\hline & $75 \%$ & 0.7566 & 0.7229 & 0.8144 & 0.7566 \\
\hline & $100 \%$ & 0.7566 & 0.7232 & 0.8148 & 0.7566 \\
\hline
\end{tabular}

The results of MLP for the NSL-KDD dataset are shown in Table 6.13. MLP shows 
a variation of results for different ratios. CGAN with a $50 \%$ ratio achieves the best F1score for MLP with CGAN. Also, SMOTE and ADASYN achieve their best result with $100 \%$ and $50 \%$, respectively. Among all the ratios, there is no definite pattern visible for any oversampling technique. But for all the oversampling techniques highest score is overall $1 \%$ higher than the base MLP result.

Table 6.13: Performance of MLP on Different ratios (NSL-KDD)

\begin{tabular}{|c|c|c|c|c|c|}
\hline & Accuracy & F1-score & Precision & Recall \\
\hline \multicolumn{2}{|c|}{ Original } & 0.7840 & 0.7632 & 0.7835 & 0.7840 \\
\hline \multirow{6}{*}{ CGAN } & $5 \%$ & 0.7881 & 0.7664 & 0.7872 & 0.7881 \\
\hline & $10 \%$ & 0.7788 & 0.7563 & 0.7745 & 0.7788 \\
\hline & $20 \%$ & 0.7901 & 0.7698 & 0.7851 & 0.7901 \\
\hline & $50 \%$ & 0.7941 & 0.7721 & 0.7946 & 0.7941 \\
\hline & $75 \%$ & 0.7868 & 0.7624 & 0.7804 & 0.7868 \\
\hline & $100 \%$ & 0.7799 & 0.7554 & 0.7895 & 0.7799 \\
\hline \multirow{6}{*}{ SMOTE } & $5 \%$ & 0.7925 & 0.7680 & 0.7942 & 0.7925 \\
\hline & $10 \%$ & 0.7888 & 0.7666 & 0.7843 & 0.7888 \\
\hline & $20 \%$ & 0.7966 & 0.7747 & 0.8061 & 0.7966 \\
\hline & $50 \%$ & 0.7892 & 0.7634 & 0.8018 & 0.7892 \\
\hline & $75 \%$ & 0.7992 & 0.7740 & 0.8065 & 0.7992 \\
\hline & $100 \%$ & 0.7955 & 0.7751 & 0.7962 & 0.7955 \\
\hline \multirow{6}{*}{ ADASYN } & $5 \%$ & 0.7907 & 0.7693 & 0.7880 & 0.7907 \\
\hline & $10 \%$ & 0.7888 & 0.7673 & 0.7912 & 0.7888 \\
\hline & $20 \%$ & 0.7908 & 0.7642 & 0.7955 & 0.7908 \\
\hline & $50 \%$ & 0.7960 & 0.7739 & 0.7975 & 0.7960 \\
\hline & $75 \%$ & 0.7944 & 0.7722 & 0.7913 & 0.7944 \\
\hline & $100 \%$ & 0.7992 & 0.7768 & 0.7979 & 0.7992 \\
\hline
\end{tabular}

LR is one of the best-performing classifiers for the AWID dataset. It is interesting to see LR performance on different ratios for the NSL-KDD dataset in Table 6.14. 
LR with CGAN has the same results for all of the ratios. It clearly shows no impact of the oversampling technique. Further investigation is done based on the confusion matrices. The prediction rate of $\mathrm{R} 2 \mathrm{~L}$ and $\mathrm{U} 2 \mathrm{R}$ decreases, and the false-negative rate of normal classes increases significantly. This increases the performance of the normal detection rate. Improvement on the normal class detection and degradation of the minority class detection keeps the result close to the original result. On the other hand, SMOTE and ADASYN do well, increasing the performance of the LR. Both of them perform the best with a $100 \%$ ratio. Their highest F1-scores are 0.7476 and 0.7797 for SMOTE and ADASYN. These results are $4.17 \%$ and $7.38 \%$ higher than the original result. LR is the only classifier whose performance increases so sharply for the oversampling techniques. In particular, LR with ADASYN shows a large variation across the different ratios. This demonstrates that LR with ADASYN is highly sensitive to the selection of the number of synthetic samples.

The above mentioned results were obtained by oversampling R2L and U2R only. We also run similar experiments for two other selections of classes. In one, we just oversampled all of the four minority classes, and for the other, we only oversampled DoS and Probe. We provide a summary of the results from these additional experiments in Table 6.15. In this table, 'R2L \& U2R' refers to oversampling on R2L and U2R only. 'ALL' denotes oversampling on all 4 minority classes. 'Probe \& DoS' refers to oversampling on Probe and DoS. We have tested the additional class selections only on NB, RF, MLP, LR. We have decided not to include AE-RL as it is an algorithmlevel approach that addresses the imbalance problem. The only thing that AE-RL lacks is the lack of a sufficient number of unique samples from the target distribution, which is being satisfied using oversampling techniques. And this lack of a sufficient number of unique samples is only observed in R2L and U2R classes. Probe and DoS have a sufficient number of samples, making it illogical to apply oversampling techniques on DoS and Probe. Since CGAN performed poorly, we have taken only the 
Table 6.14: Performance of LR on Different ratios (NSL-KDD)

\begin{tabular}{|c|c|l|l|l|l|}
\hline \multicolumn{2}{|c|}{} & Accuracy & F1-score & Precision & Recall \\
\hline \multicolumn{1}{|c|}{ Original } & 0.7499 & 0.7059 & 0.7650 & 0.7499 \\
\hline \multirow{5}{*}{ CGAN } & $5 \%$ & 0.7499 & $\mathbf{0 . 7 0 6 1}$ & 0.7610 & 0.7499 \\
\cline { 2 - 6 } & $10 \%$ & 0.7499 & $\mathbf{0 . 7 0 6 1}$ & 0.7607 & 0.7499 \\
\cline { 2 - 6 } & $20 \%$ & 0.7499 & $\mathbf{0 . 7 0 6 1}$ & 0.7602 & 0.7499 \\
\cline { 2 - 6 } & $50 \%$ & 0.7499 & $\mathbf{0 . 7 0 6 1}$ & 0.7598 & 0.7499 \\
\cline { 2 - 6 } & $75 \%$ & 0.7499 & $\mathbf{0 . 7 0 6 1}$ & 0.7599 & 0.7499 \\
\cline { 2 - 6 } & $100 \%$ & 0.7499 & $\mathbf{0 . 7 0 6 1}$ & 0.7597 & 0.7499 \\
\hline \multirow{5}{*}{ SMOTE } & $5 \%$ & 0.7626 & 0.7332 & 0.8052 & 0.7626 \\
\cline { 2 - 6 } & $10 \%$ & 0.7630 & 0.7351 & 0.8031 & 0.7630 \\
\cline { 2 - 6 } & $20 \%$ & 0.7634 & 0.7404 & 0.8018 & 0.7634 \\
\cline { 2 - 6 } & $50 \%$ & 0.7646 & 0.7435 & 0.8013 & 0.7646 \\
\cline { 2 - 6 } & $75 \%$ & 0.7656 & 0.7457 & 0.8008 & 0.7656 \\
\cline { 2 - 6 } & $100 \%$ & 0.7665 & $\mathbf{0 . 7 4 7 6}$ & 0.7985 & 0.7665 \\
\hline \multirow{5}{*}{ ADASYN } & $5 \%$ & 0.7611 & 0.7331 & 0.7883 & 0.7611 \\
\cline { 2 - 6 } & $10 \%$ & 0.7633 & 0.7414 & 0.7911 & 0.7633 \\
\cline { 2 - 6 } & $20 \%$ & 0.7655 & 0.7473 & 0.7899 & 0.7655 \\
\cline { 2 - 6 } & $50 \%$ & 0.7718 & 0.7596 & 0.7953 & 0.7718 \\
\hline & $75 \%$ & 0.7820 & 0.7729 & 0.8036 & 0.7820 \\
\hline & $100 \%$ & 0.7878 & $\mathbf{0 . 7 7 9 7}$ & 0.8082 & 0.7878 \\
\hline
\end{tabular}

results of SMOTE and ADASYN, over all ratios, for calculating the average F1-score per classifier. The rows marked "Best" indicate the best achieved F1-score per classifier from SMOTE and ADASYN results. LR, MLP, and RF have almost the same average F1-score for both R2L \& U2R and all four classes. The Probe \& DoS category always displays inferior performance compared to the other two categories. The best result supports this claim by having less F1-score for the best result when only Probe and DoS are being oversampled. From these results, it is clear that oversampling only 
Table 6.15: Results Summary on Three Different Combinations of Oversampling Techniques

\begin{tabular}{|c|l|l|l|l|}
\hline \multicolumn{2}{|c|}{} & R2L \& U2R & ALL & Probe \& DoS \\
\hline \multirow{2}{*}{ NB } & AVERAGE & 0.6185 & 0.6190 & 0.6245 \\
\cline { 2 - 5 } & BEST & 0.6360 & 0.6369 & 0.6472 \\
\hline \multirow{2}{*}{ RF } & AVERAGE & 0.7234 & 0.7243 & 0.7121 \\
\cline { 2 - 5 } & BEST & 0.7287 & 0.7320 & 0.7185 \\
\hline \multirow{2}{*}{ MLP } & AVERAGE & 0.7234 & 0.7243 & 0.7121 \\
\cline { 2 - 5 } & BEST & 0.7768 & 0.7847 & 0.7639 \\
\hline \multirow{2}{*}{ LR } & AVERAGE & 0.7705 & 0.7707 & 0.7587 \\
\cline { 2 - 5 } & BEST & 0.7797 & 0.7814 & 0.7172 \\
\hline
\end{tabular}

on Probe and DoS does not help that much. Furthermore, oversampling on all the four classes and only on R2L \& U2R produces very similar results to each other. It is more efficient to oversample fewer classes in terms of computational time, resources, variances. Based on these results, we think it is effective to oversample only on R2L and $\mathrm{U} 2 \mathrm{R}$ in order to solve the imbalance problem of the dataset.

\subsection{CGAN Performances on Both Datasets}

Proposing CGAN as an oversampling technique and combining it with other classifiers to enhance performance is one of the key contributions in this dissertation. But CGAN itself has its disadvantages. We have tested CGAN with two different datasets. One of the main differences between these two datasets is that one is highly imbalanced, and another is less imbalanced in terms of the number of samples. Though the AWID dataset is highly imbalanced, its minority classes have ample samples to offer a good representation of the class distribution. In comparison, NSL-KDD is less imbalanced than the AWID dataset but has very few samples in the minority classes, and there are 
also a good amount of unseen samples of subclasses in the testing dataset from those minority classes. CGAN requires enough data to train effectively. It will only work effectively when it has sufficient data to mimic the class distribution. It is one of the main reasons CGAN is performing as the best oversampling technique in the AWID dataset and performs worst in the NSL-KDD dataset. We recommend not to use CGAN for the dataset with extremely low samples in the minority classes. CGAN will be an appropriate choice for those datasets where there are enough samples present in the minority classes. Another benefit of CGAN is it performs well with AE-RL. Our proposed algorithm AEGAN performs well for both datasets and achieves better than the original dataset with its corresponding ratio. Though the synthetic sample generated by CGAN are of poor quality in the NSL-KDD, it is enough to create variations that help AE-RL improve its performance. 


\section{Chapter 7}

\section{Conclusion and Future Work}

In this dissertation, we first conducted a comparative evaluation study on an imbalanced dataset using ten machine learning algorithms combined with three different resampling techniques to detect network intrusion. Among these ten classifiers, we proposed a customized voting classifier that uses 9 different standard machine learning and deep learning algorithms. The advantage of the proposed voting classifier is evident when all participating classifiers perform well to detect individual classes. In most cases, it yields better results when running on resampled datasets. In general, the weaker learners' bad performance is overruled after the majority voting process. However, certain classifiers are only good at predicting certain classes. Therefore, giving all classifiers' decisions an equal weight might restrict the potential performance of the voting classifier. Consequently, we plan to work on a weighted voting system to improve the proposed voting classifier's performance. All of the experimental results are performed on the NSL-KDD dataset. Based on the experimental results, Deep learning models tend to suffer when undersampling techniques are applied to the dataset. Our intuition is that changing the architecture of deep learning models may lead to a better result. We also observe that resampling techniques have a positive impact on the performance of the Standard Machine Learning Algorithm. In general, the oversampling approach shows better performance than the other two 
resampling techniques. And among the oversampling techniques, CGAN performs the best compared to the other oversampling techniques for all studied classifiers.

Then, we turned our attention to the impact of the oversampling technique in network intrusion detection systems. We proposed an IDS architecture that combines a CGAN-based oversampling technique with AE-RL. We also implemented the combination of a CGAN-based oversampling technique with other classifiers as well. To extend the comparative evaluation on oversampling technique, we compared CGAN with two other oversampling techniques: SMOTE and ADASYN. All the experimental models were evaluated on the AWID dataset. Based on the experimental results, we provided a thorough performance analysis to display the benefit of proposed models. Results demonstrate that LR combined with CGAN achieves the best result in terms of F1-score compared to all other classifier-oversampling combinations. The algorithm-level approach AE-RL shows the least amount of variation when combined with oversampling techniques. This suggests there may be limited benefit in the combination of data-level approaches with algorithm-level approaches. Expansion of the experiments to include additional algorithm-level approaches is required to further investigate this topic. Overall, most data-level approaches have a positive effect on the performance of classifiers.

Finally, we further extended our experimental methodology in order to provide a more robust set of experiments. We validated oversampling techniques on two different types of datasets. We proposed a refined AE-RL data selection technique over the existing one in AE-RL architecture. This refined selection technique is crucial when AE-RL is used in combination with other oversampling techniques on datasets like NSL-KDD that have extremely low number of samples in its training data. A comparative evaluation was conducted between CGAN and WCGAN where CGAN proved to be a better choice as a GAN-based oversampling technique. In the future, we plan to investigate GAN variants that can possibly be used as an oversampling 
technique. Also, we demonstrated how one of the CGAN's hyperparameters, Generator Steps (GS), impacts the training process of CGAN. GS $=2$ works the best for the AWID dataset, and GS = 1 yields the best performance on the NSL-KDD dataset. We further examined the impact of the amount of synthetic samples to be generated by each of these three oversampling techniques, CGAN, SMOTE and ADASYN, on the performance of different classifiers. Experiments were done on both the AWID and NSL-KDD datasets. However, we observed no consistent trend on these classifier-oversampling combinations when different oversampled-minorityclass/Majority-Class sample-ratios were used. Additionally, we conducted an experimental analysis on the choice of the minority classes to be oversampled on NSL-KDD, where no unique distribution exists in its minority classes. Based on the results, we conclude that oversampling only R2L and U2R that are the smallest minority classes instead of oversampling all four minority classes yields a better result. Lastly, we discussed the performance of CGAN on two different datasets. The results show that CGAN's performance on network intrusion detection mainly depends on the dataset. If a dataset has extremely low samples in its minority classes, CGAN is not an ideal choice as an oversampling technique. In the immediate future, we plan to carry out a sensitivity analysis on the impact of various oversampled-minority-class/MajorityClass sample-ratios on the list of classifier-oversampling combinations. The results of this analysis should give us more insight on which combination(s) is more sensitive to the change of ratios. The impact of Dimensional Reduction on the classifieroversampling combinations should also be an interesting direction to explore. 


\section{List of References}

[1] M. H. Bhuyan, D. K. Bhattacharyya, and J. K. Kalita, "Towards generating real-life datasets for network intrusion detection.," IJ Network Security, vol. 17, no. 6, pp. 683-701, 2015.

[2] J. Jang-Jaccard and S. Nepal, "A survey of emerging threats in cybersecurity," Journal of Computer and System Sciences, vol. 80, no. 5, pp. 973-993, 2014.

[3] H. A. M. Uppal, M. Javed, and M. Arshad, "An overview of intrusion detection system (ids) along with its commonly used techniques and classifications," International Journal of Computer Science and Telecommunications, vol. 5, no. 2, pp. 20-24, 2014.

[4] N. Sun, J. Zhang, P. Rimba, S. Gao, L. Y. Zhang, and Y. Xiang, "Data-driven cybersecurity incident prediction: A survey," IEEE communications surveys 83 tutorials, vol. 21, no. 2, pp. 1744-1772, 2018.

[5] P. Mishra, V. Varadharajan, U. Tupakula, and E. S. Pilli, "A detailed investigation and analysis of using machine learning techniques for intrusion detection," IEEE Communications Surveys \& Tutorials, vol. 21, no. 1, pp. 686-728, 2018.

[6] A. Khraisat, I. Gondal, P. Vamplew, and J. Kamruzzaman, "Survey of intrusion detection systems: techniques, datasets and challenges," Cybersecurity, vol. 2, no. 1, pp. 1-22, 2019.

[7] A. Torkaman, G. Javadzadeh, and M. Bahrololum, "A hybrid intelligent hids model using two-layer genetic algorithm and neural network," in The 5th Conference on Information and Knowledge Technology, pp. 92-96, IEEE, 2013.

[8] M. Liu, Z. Xue, X. Xu, C. Zhong, and J. Chen, "Host-based intrusion detection system with system calls: Review and future trends," ACM Computing Surveys (CSUR), vol. 51, no. 5, pp. 1-36, 2018. 
[9] U. H. Rao and U. Nayak, "Intrusion detection and prevention systems," in The InfoSec Handbook, pp. 225-243, Springer, 2014.

[10] R. Puzis, M. D. Klippel, Y. Elovici, and S. Dolev, "Optimization of nids placement for protection of intercommunicating critical infrastructures," in European Conference on Intelligence and Security Informatics, pp. 191-203, Springer, 2008.

[11] M. H. Bhuyan, D. K. Bhattacharyya, and J. K. Kalita, "Network anomaly detection: methods, systems and tools," IEEE Communications Surveys $\&$ Tutorials, vol. 16, no. 1, pp. 303-336, 2013.

[12] A. Azab, M. Alazab, and M. Aiash, "Machine learning based botnet identification traffic," in 2016 IEEE Trustcom/BigDataSE/ISPA, pp. 1788-1794, IEEE, 2016.

[13] R. Sommer and V. Paxson, "Outside the closed world: On using machine learning for network intrusion detection," in 2010 IEEE symposium on security and privacy, pp. 305-316, IEEE, 2010.

[14] N. Japkowicz, "The class imbalance problem: Significance and strategies," in Proc. of the Int'l Conf. on Artificial Intelligence, vol. 56, Citeseer, 2000.

[15] N. V. Chawla, K. W. Bowyer, L. O. Hall, and W. P. Kegelmeyer, "Smote: synthetic minority over-sampling technique," Journal of artificial intelligence research, vol. 16, pp. 321-357, 2002.

[16] X.-Y. Liu, J. Wu, and Z.-H. Zhou, "Exploratory undersampling for classimbalance learning," IEEE Transactions on Systems, Man, and Cybernetics, Part B (Cybernetics), vol. 39, no. 2, pp. 539-550, 2008.

[17] G. E. Batista, R. C. Prati, and M. C. Monard, "A study of the behavior of several methods for balancing machine learning training data," ACM SIGKDD explorations newsletter, vol. 6, no. 1, pp. 20-29, 2004.

[18] H. Liu and B. Lang, "Machine learning and deep learning methods for intrusion detection systems: A survey," applied sciences, vol. 9, no. 20, p. 4396, 2019.

[19] F. Kuang, W. Xu, and S. Zhang, "A novel hybrid kpca and svm with ga model for intrusion detection," Applied Soft Computing, vol. 18, pp. 178-184, 2014.

[20] R. R. Reddy, Y. Ramadevi, and K. N. Sunitha, "Effective discriminant function for intrusion detection using svm," in 2016 International conference on advances 
in computing, communications and informatics (ICACCI), pp. 1148-1153, IEEE, 2016.

[21] N. Farnaaz and M. Jabbar, "Random forest modeling for network intrusion detection system," Procedia Computer Science, vol. 89, pp. 213-217, 2016.

[22] J. Zhang, M. Zulkernine, and A. Haque, "Random-forests-based network intrusion detection systems," IEEE Transactions on Systems, Man, and Cybernetics, Part C (Applications and Reviews), vol. 38, no. 5, pp. 649-659, 2008.

[23] W. Li, P. Yi, Y. Wu, L. Pan, and J. Li, "A new intrusion detection system based on knn classification algorithm in wireless sensor network," Journal of Electrical and Computer Engineering, vol. 2014, 2014.

[24] B. Ingre and A. Yadav, "Performance analysis of nsl-kdd dataset using ann," in 2015 international conference on signal processing and communication engineering systems, pp. 92-96, IEEE, 2015.

[25] G. Caminero, M. Lopez-Martin, and B. Carro, "Adversarial environment reinforcement learning algorithm for intrusion detection," Computer Networks, vol. 159, pp. 96-109, 2019.

[26] I. J. Goodfellow, J. Pouget-Abadie, M. Mirza, B. Xu, D. Warde-Farley, S. Ozair, A. Courville, and Y. Bengio, "Generative adversarial networks," arXiv preprint arXiv:1406.2661, 2014.

[27] C. Esteban, S. L. Hyland, and G. Rätsch, "Real-valued (medical) time series generation with recurrent conditional gans," arXiv preprint arXiv:1706.02633, 2017.

[28] M. Mirza and S. Osindero, "Conditional generative adversarial nets," arXiv preprint arXiv:1411.1784, 2014.

[29] O. Simeone, "A very brief introduction to machine learning with applications to communication systems," IEEE Transactions on Cognitive Communications and Networking, vol. 4, no. 4, pp. 648-664, 2018.

[30] S. Uddin, A. Khan, M. E. Hossain, and M. A. Moni, "Comparing different supervised machine learning algorithms for disease prediction," BMC medical informatics and decision making, vol. 19, no. 1, pp. 1-16, 2019. 
[31] S. Kanchanasuk and K. Sinapiromsaran, "Recursive binary tube partitioning for classification," in Intelligent and Evolutionary Systems, pp. 99-107, Springer, 2016.

[32] P. C. Sen, M. Hajra, and M. Ghosh, "Supervised classification algorithms in machine learning: A survey and review," in Emerging technology in modelling and graphics, pp. 99-111, Springer, 2020.

[33] P. Geurts, D. Ernst, and L. Wehenkel, "Extremely randomized trees," Machine learning, vol. 63, no. 1, pp. 3-42, 2006.

[34] F. Pedregosa, G. Varoquaux, A. Gramfort, V. Michel, B. Thirion, O. Grisel, M. Blondel, P. Prettenhofer, R. Weiss, V. Dubourg, et al., "Scikit-learn: Machine learning in python," the Journal of machine Learning research, vol. 12, pp. 28252830, 2011.

[35] I. Goodfellow, Y. Bengio, A. Courville, and Y. Bengio, Deep learning, vol. 1. MIT press Cambridge, 2016.

[36] R. S. Sutton, "Introduction: The challenge of reinforcement learning," in Reinforcement Learning, pp. 1-3, Springer, 1992.

[37] X. Ma and W. Shi, "AESMOTE: Adversarial reinforcement learning with smote for anomaly detection," IEEE Transactions on Network Science and Engineering, 2020.

[38] J. Kong, T. Rios, W. Kowalczyk, S. Menzel, and T. Bäck, "On the performance of oversampling techniques for class imbalance problems," in Pacific-Asia Conference on Knowledge Discovery and Data Mining, pp. 84-96, Springer, 2020.

[39] H. He, Y. Bai, E. A. Garcia, and S. Li, "ADASYN: Adaptive synthetic sampling approach for imbalanced learning," in 2008 IEEE international joint conference on neural networks (IEEE world congress on computational intelligence), pp. 1322-1328, IEEE, 2008.

[40] U. Fiore, A. De Santis, F. Perla, P. Zanetti, and F. Palmieri, "Using generative adversarial networks for improving classification effectiveness in credit card fraud detection," Information Sciences, vol. 479, pp. 448-455, 2019.

[41] G. Douzas and F. Bacao, "Effective data generation for imbalanced learning using conditional generative adversarial networks," Expert Systems with applications, vol. 91, pp. 464-471, 2018. 
[42] S.-J. Yen and Y.-S. Lee, "Under-sampling approaches for improving prediction of the minority class in an imbalanced dataset," in Intelligent Control and Automation, pp. 731-740, Springer, 2006.

[43] D. L. Wilson, "Asymptotic properties of nearest neighbor rules using edited data," IEEE Transactions on Systems, Man, and Cybernetics, no. 3, pp. 408$421,1972$.

[44] C. Kolias, G. Kambourakis, A. Stavrou, and S. Gritzalis, "Intrusion detection in 802.11 networks: empirical evaluation of threats and a public dataset," IEEE Communications Surveys $\&$ Tutorials, vol. 18, no. 1, pp. 184-208, 2015.

[45] M. Tavallaee, E. Bagheri, W. Lu, and A. A. Ghorbani, "A detailed analysis of the kdd cup 99 data set," in 2009 IEEE symposium on computational intelligence for security and defense applications, pp. 1-6, IEEE, 2009.

[46] R. Vinayakumar, M. Alazab, K. Soman, P. Poornachandran, A. Al-Nemrat, and S. Venkatraman, "Deep learning approach for intelligent intrusion detection system," IEEE Access, vol. 7, pp. 41525-41550, 2019.

[47] C. Yin, Y. Zhu, J. Fei, and X. He, "A deep learning approach for intrusion detection using recurrent neural networks," Ieee Access, vol. 5, pp. 21954-21961, 2017.

[48] M. Lopez-Martin, B. Carro, A. Sanchez-Esguevillas, and J. Lloret, "Conditional variational autoencoder for prediction and feature recovery applied to intrusion detection in iot," Sensors, vol. 17, no. 9, p. 1967, 2017.

[49] M. Lopez-Martin, B. Carro, and A. Sanchez-Esguevillas, "Variational data generative model for intrusion detection," Knowledge and Information Systems, vol. 60, no. 1, pp. 569-590, 2019.

[50] A. K. Shrivas and A. K. Dewangan, "An ensemble model for classification of attacks with feature selection based on kdd99 and nsl-kdd data set," International Journal of Computer Applications, vol. 99, no. 15, pp. 8-13, 2014.

[51] Y. Zhou, G. Cheng, S. Jiang, and M. Dai, "An efficient intrusion detection system based on feature selection and ensemble classifier," arXiv preprint arXiv:1904.01352, 2019.

[52] X. Gao, C. Shan, C. Hu, Z. Niu, and Z. Liu, "An adaptive ensemble machine learning model for intrusion detection," IEEE Access, vol. 7, pp. 82512-82521, 2019. 
[53] M. Almseidin, M. Alzubi, S. Kovacs, and M. Alkasassbeh, "Evaluation of machine learning algorithms for intrusion detection system," in 2017 IEEE 15th International Symposium on Intelligent Systems and Informatics (SISY), pp. 000277000282, IEEE, 2017.

[54] Z. He, T. Zhang, and R. B. Lee, "Machine learning based ddos attack detection from source side in cloud," in 2017 IEEE 4th International Conference on Cyber Security and Cloud Computing (CSCloud), pp. 114-120, IEEE, 2017.

[55] Y. Wahba, E. ElSalamouny, and G. ElTaweel, "Improving the performance of multi-class intrusion detection systems using feature reduction," arXiv preprint arXiv:150\%.06692, 2015.

[56] A. Husain, A. Salem, C. Jim, and G. Dimitoglou, "Development of an efficient network intrusion detection model using extreme gradient boosting (xgboost) on the unsw-nb15 dataset," in 2019 IEEE International Symposium on Signal Processing and Information Technology (ISSPIT), pp. 1-7, IEEE, 2019.

[57] R. Abdulhammed, M. Faezipour, A. Abuzneid, and A. Alessa, "Enhancing wireless intrusion detection using machine learning classification with reduced attribute sets," in 2018 14th International Wireless Communications 85 Mobile Computing Conference (IWCMC), pp. 524-529, IEEE, 2018.

[58] M. N. Chowdhury, A support vector machine cost function in simulated annealing for network intrusion detection. PhD thesis, University of Manitoba, 2019.

[59] S. Bhattacharya, P. K. R. Maddikunta, R. Kaluri, S. Singh, T. R. Gadekallu, M. Alazab, U. Tariq, et al., "A novel pca-firefly based xgboost classification model for intrusion detection in networks using gpu," Electronics, vol. 9, no. 2, p. 219, 2020.

[60] V. L. Thing, "IEEE 802.11 network anomaly detection and attack classification: A deep learning approach," in 2017 IEEE Wireless Communications and Networking Conference (WCNC), pp. 1-6, IEEE, 2017.

[61] Z. Li, Z. Qin, K. Huang, X. Yang, and S. Ye, "Intrusion detection using convolutional neural networks for representation learning," in International conference on neural information processing, pp. 858-866, Springer, 2017.

[62] W. Elmasry, A. Akbulut, and A. H. Zaim, "Empirical study on multiclass classification-based network intrusion detection," Computational Intelligence, vol. 35, no. 4, pp. 919-954, 2019. 
[63] G. E. Hinton and R. R. Salakhutdinov, "Reducing the dimensionality of data with neural networks," science, vol. 313, no. 5786, pp. 504-507, 2006.

[64] Z. Chen, C. K. Yeo, B. S. Lee, and C. T. Lau, "Autoencoder-based network anomaly detection," in 2018 Wireless Telecommunications Symposium (WTS), pp. 1-5, IEEE, 2018.

[65] M. Lopez-Martin, B. Carro, and A. Sanchez-Esguevillas, "Application of deep reinforcement learning to intrusion detection for supervised problems," Expert Systems with Applications, vol. 141, p. 112963, 2020.

[66] R. Abdulhammed, M. Faezipour, A. Abuzneid, and A. AbuMallouh, "Deep and machine learning approaches for anomaly-based intrusion detection of imbalanced network traffic," IEEE sensors letters, vol. 3, no. 1, pp. 1-4, 2018.

[67] G. Karatas, O. Demir, and O. K. Sahingoz, "Increasing the performance of machine learning-based idss on an imbalanced and up-to-date dataset," IEEE Access, vol. 8, pp. 32150-32162, 2020.

[68] P.-J. Chuang and D.-Y. Wu, "Applying deep learning to balancing network intrusion detection datasets," in 2019 IEEE 11th International Conference on Advanced Infocomm Technology (ICAIT), pp. 213-217, IEEE, 2019.

[69] L. Liu, P. Wang, J. Lin, and L. Liu, "Intrusion detection of imbalanced network traffic based on machine learning and deep learning," IEEE Access, 2020.

[70] H. Zhang, L. Huang, C. Q. Wu, and Z. Li, "An effective convolutional neural network based on smote and gaussian mixture model for intrusion detection in imbalanced dataset," Computer Networks, vol. 177, p. 107315, 2020.

[71] S. Bagui and K. Li, "Resampling imbalanced data for network intrusion detection datasets," Journal of Big Data, vol. 8, no. 1, pp. 1-41, 2021.

[72] L. Vu, C. T. Bui, and Q. U. Nguyen, "A deep learning based method for handling imbalanced problem in network traffic classification," in Proceedings of the Eighth International Symposium on Information and Communication Technology, pp. 333-339, 2017.

[73] M. Zheng, T. Li, R. Zhu, Y. Tang, M. Tang, L. Lin, and Z. Ma, "Conditional wasserstein generative adversarial network-gradient penalty-based approach to alleviating imbalanced data classification," Information Sciences, vol. 512, pp. 1009-1023, 2020. 
[74] J.-H. Oh, J. Y. Hong, and J.-G. Baek, "Oversampling method using outlier detectable generative adversarial network," Expert Systems with Applications, vol. 133, pp. 1-8, 2019.

[75] Y. Liu, Z. Li, C. Zhou, Y. Jiang, J. Sun, M. Wang, and X. He, "Generative adversarial active learning for unsupervised outlier detection," IEEE Transactions on Knowledge and Data Engineering, vol. 32, no. 8, pp. 1517-1528, 2019.

[76] S. Huang and K. Lei, "Igan-ids: An imbalanced generative adversarial network towards intrusion detection system in ad-hoc networks," Ad Hoc Networks, vol. 105, p. 102177, 2020.

[77] J. Lee and K. Park, "Ae-cgan model based high performance network intrusion detection system," Applied Sciences, vol. 9, no. 20, p. 4221, 2019.

[78] Y. Yang, K. Zheng, B. Wu, Y. Yang, and X. Wang, "Network intrusion detection based on supervised adversarial variational auto-encoder with regularization," IEEE Access, vol. 8, pp. 42169-42184, 2020.

[79] L. Gnanaprasanambikai and N. Munusamy, "Data pre-processing and classification for traffic anomaly intrusion detection using nslkdd dataset," Cybernetics and Information Technologies, vol. 18, no. 3, pp. 111-119, 2018.

[80] I. Mani and I. Zhang, "knn approach to unbalanced data distributions: a case study involving information extraction," in Proceedings of workshop on learning from imbalanced datasets, vol. 126, ICML United States, 2003.

[81] D. S. Berman, A. L. Buczak, J. S. Chavis, and C. L. Corbett, "A survey of deep learning methods for cyber security," Information, vol. 10, no. 4, p. 122, 2019.

[82] F. Chollet et al., "Keras." https://keras.io, 2015.

[83] M. Arjovsky, S. Chintala, and L. Bottou, "Wasserstein generative adversarial networks," in Proceedings of the 34th International Conference on Machine Learning, ICML 2017, Sydney, NSW, Australia, 6-11 August 2017 (D. Precup and Y. W. Teh, eds.), vol. 70 of Proceedings of Machine Learning Research, pp. 214223, PMLR, 2017. 